\title{
Toxicology and pharmacology of synthetic organoselenium compounds: an update
}

\author{
Cristina W. Nogueira ${ }^{1}$ (D) Nilda V. Barbosa ${ }^{1} \cdot$ João B. T. Rocha ${ }^{1}$
}

Received: 5 January 2021 / Accepted: 10 February 2021 / Published online: 1 April 2021

(c) The Author(s), under exclusive licence to Springer-Verlag GmbH, DE part of Springer Nature 2021

\begin{abstract}
Here, we addressed the pharmacology and toxicology of synthetic organoselenium compounds and some naturally occurring organoselenium amino acids. The use of selenium as a tool in organic synthesis and as a pharmacological agent goes back to the middle of the nineteenth and the beginning of the twentieth centuries. The rediscovery of ebselen and its investigation in clinical trials have motivated the search for new organoselenium molecules with pharmacological properties. Although ebselen and diselenides have some overlapping pharmacological properties, their molecular targets are not identical. However, they have similar anti-inflammatory and antioxidant activities, possibly, via activation of transcription factors, regulating the expression of antioxidant genes. In short, our knowledge about the pharmacological properties of simple organoselenium compounds is still elusive. However, contrary to our early expectations that they could imitate selenoproteins, organoselenium compounds seem to have non-specific modulatory activation of antioxidant pathways and specific inhibitory effects in some thiol-containing proteins. The thiol-oxidizing properties of organoselenium compounds are considered the molecular basis of their chronic toxicity; however, the acute use of organoselenium compounds as inhibitors of specific thiol-containing enzymes can be of therapeutic significance. In summary, the outcomes of the clinical trials of ebselen as a mimetic of lithium or as an inhibitor of SARS-CoV-2 proteases will be important to the field of organoselenium synthesis. The development of computational techniques that could predict rational modifications in the structure of organoselenium compounds to increase their specificity is required to construct a library of thiol-modifying agents with selectivity toward specific target proteins.
\end{abstract}

Keywords Ebselen $\cdot$ Selenium $\cdot$ Diselenides $\cdot$ Toxicology $\cdot$ Pharmacology $\cdot$ Thiol

\section{Introduction}

In this review, we shall cover toxicological and pharmacological effects, in which organoselenium compounds are involved, but the effects of inorganic compounds will not be addressed here. The review mostly discusses recent literature, starting from 2011 until the end of 2020; however, some earlier studies are cited when needed. Method data for this review were sourced from online Web of Science database. The deadline for data search was August 2020; no

Cristina W. Nogueira

criswn@ufsm.br

$\triangle$ João B. T. Rocha

jbtrocha@yahoo.com.br

1 Laboratório de Síntese, Reatividade e Avaliação Farmacológica E Toxicológica de Organocalcogênios, Centro de Ciências Naturais E Exatas, Universidade Federal de Santa Maria, Santa Maria, RS CEP 97105-900, Brazil data were excluded based on language or publication origin. Since, it is not possible to cite all of the findings that have taken place, we apologize to those whose work has been omitted.

The chemical structures of representative organoselenium compounds which will be discussed in this review are shown in Scheme 1.

\section{A brief history of selenium: an element with two faces}

Since its discovery about 200 years ago, selenium has been attracting the interest of chemists and biologists. Soon after its isolation by Jacobs Berzelius, in 1817, selenium was used in organic synthesis and investigated as a potentially toxic or beneficial agent both in animals (including humans) and plants (Levine 1915, 1925; Martin 1936; Rocha et al. 2017; Smith 1941; Weil 1915). One of the first therapeutic uses of selenium was in the treatment of cancer and reports about 
Scheme 1 Chemical structures of representative organoselenium compounds discussed in this review

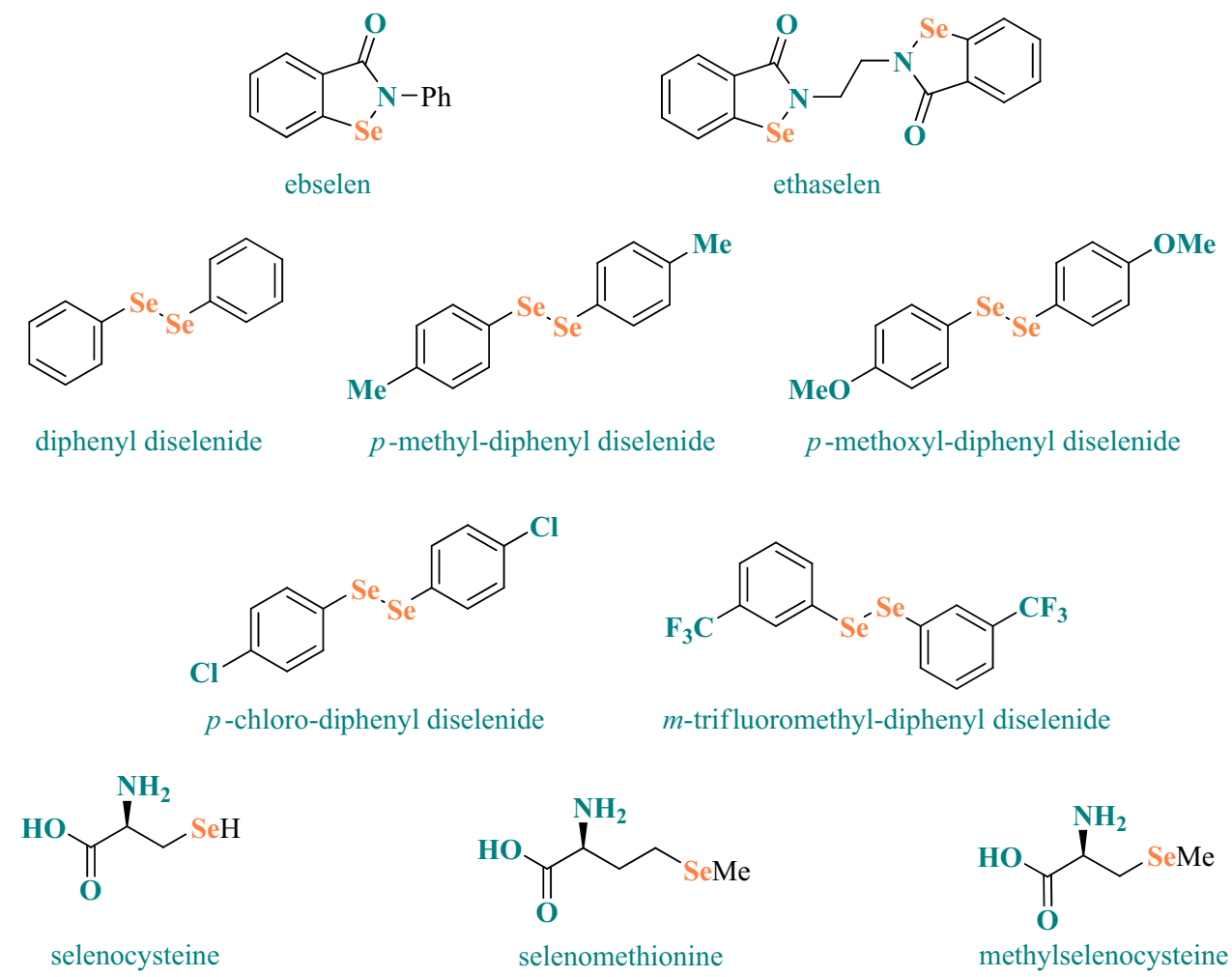

the beneficial effects of elemental selenium in the treatment of inoperable carcinoma can be found in clinical studies published at the beginning of the twentieth century (Freeman 1922; Watson-Williams 1919; Weil 1915). In sharp contrast, the lethal effect of a single injection of selenite in one human patient with cancer can also be found in the literature, cited in Weil (1915). Subsequently, selenium was rarely used in cancer treatment in humans, possibly because its effectiveness in clinical studies was inconsistent (Weil 1915). In addition, the toxicity of selenium became notorious in farm and experimental animals exposed to high levels of the element (Levine 1915, 1925; Painter 1941; Smith 1941). In short, the early history of the element selenium in biology has been marked by the contrast between its toxic and beneficial effects.

The importance of selenium to mammals started to be defined in 1957 when Schwartz and Foltz demonstrated that selenite and selenate could prevent liver necrosis caused by feeding a vitamin E-deficient diet to rats. Though the classical paper of Schwartz and Foltz did not establish dietary essentiality to selenium in rats, it gave the first demonstration that selenium could mitigate the deficiency of an essential vitamin (Schwarz and Foltz 1957). The explanation on how vitamin $\mathrm{E}$ and selenium have partial overlapping nutritional and biochemical protective effects in mammals was deciphered only in 1985, when the phospholipid hydroperoxide glutathione peroxidase (GPx4) was characterized as a selenium enzyme (Ursini et al. 1985). GPx4 is involved in the degradation of phospholipid hydroperoxides in biomembranes of mammalian cells, and consequently, it protects cell membranes from lipid peroxidation up-stream to vitamin E. Vitamin E scavenges phospholipid hydroperoxyl radicals directly, whereas GPx4 decreases the concentration of phospholipid peroxides that can generate the reactive peroxyl radicals. It is noteworthy that the selenium atom of the selenol (-SeH) group of GPx4 interacts directly with lipid peroxides in biomembranes, whereas vitamin $\mathrm{E}$ interacts with lipid peroxide radicals.

The first discovered biochemical role of selenium was its presence as an integral part of the enzyme glutathione peroxidase (Flohe et al. 1973; Rotruck et al. 1973). The accurate molecular role played by selenium in mammalian cell biochemistry was elucidated in 1978, when the chemical nature of selenium, in the active site of rat liver glutathione peroxidase, was deciphered (Forstrom et al. 1978). The studies of Cone with bacteria (Cone et al. 1976) and Forstrom with rodents (Forstrom et al. 1978) introduced in the chemistry of life a new amino acid and a new functional group: the selenocysteine and the selenol group. They are analogs of the amino acid cysteine and its functional thiol group. The essentiality of selenocysteine and its selenol group in the biochemistry and physiology of mammalian cells will be presented in the next section: selenium physiology: selenium as a component of selenoproteins.

Adequate selenium intake has also been indicated to be critical to proper immune function and decrease the risk of 
cardiovascular diseases (Avery and Hoffmann 2018; Huang et al. 2012; Kuria et al. 2020; Qian et al. 2019; Rayman 2012). Furthermore, beneficial effects of appropriate blood selenium levels as a factor against virus infections (particularly HIV and, more, recently against SARS-CoV-2) and sepsis severity have also frequently appeared in the literature (Aggarwal et al. 2016; Alhazzani et al. 2013; Guillin et al. 2019; Heller et al. 2020; Mertens et al. 2015; Moghaddam et al. 2020; Rayman 2012; Zhang et al. 2020b, c). However, negative and contradictory results can also be found in the literature (Bloos et al. 2016; Kamwesiga et al. 2015; Shivakoti et al. 2014; Stone et al. 2010). However, it is still elusive if selenium has specific direct role in such complex physiological, immunological, and pathological responses or if selenoproteins modulate indirectly the inflammatory and other responses by modulating the redox state of the body.

The influence of selenium supplementation as a potential anticarcinogenic agent was studied in a large epidemiological study in USA. The SELECT study compared the supplementation of selenium (as selenomethionine) and vitamin $\mathrm{E}$ in the incidence of prostate cancer. However, the study was interrupted before planned, because the data, contrary to the expectation, have not indicated potential beneficial effects of selenium or vitamin E. Despite the negative outcomes of SELECT (Dunn et al. 2010; Klein et al. 2011; Lippman et al. 2009; Nicastro and Dunn 2013), the potential use of selenium in cancer prevention or treatment is still a matter of debate (Chapelle et al. 2020; Vinceti et al. 2018). Several experimental studies have indicated the potential anti-cancer properties of inorganic and organic forms of selenium (Álvarez-Pérez et al. 2018; Gandin et al. 2018; Gopalakrishna et al. 2018; Krasowska et al. 2019; Ruberte et al. 2019; Sanmartín et al. 2012; Sharma and Amin 2013; Spengler et al. 2019; Steinbrenner et al. 2013; Tan et al. 2019). Some clinical studies have also demonstrated that selenite can have beneficial effects by itself or decrease some toxic effects of radiotherapy in cancer patients (Brodin et al. 2015; Han et al. 2019; Handa et al. 2020; Knox et al. 2019; Muecke et al. 2014). More recently, ethaselen (a derivative of ebselen) was described to have pharmacological effects against lung cancer cell lines and is now recruiting patients for clinical trials for lung cancer treatment (Tan et al. 2019; Zheng et al. 2017b).

The supranutritional intake of selenium has been linked with increased risk of developing type 2 diabetes, advanced prostate cancer, hypertension, dyslipidemia, and neurodegenerative diseases (amyotrophic lateral sclerosis (ALS), early onset dementia, and Parkinson Disease) (Adani et al. 2020a, b; Bastola et al. 2020; Loomba et al. 2020; Vinceti et al. 2018, 2019a, b; Wu et al. 2018; Yarmolinsky et al. 2018). In accordance with epidemiological studies, the intake of supranutritional levels of selenium, which were associated with an overexpression of two selenoenzymes [glutathione peroxidase 1 (GPx1) and methionine sulfoxide reductase (MsrB1)] has been shown to cause hyperinsulinemia and insulin resistance in mice (Labunskyy et al. 2011). The detrimental effects of selenium in glucose homeostasis have been attributed to deregulation of cell redox balance (reductive stress). In contrast, the insufficient synthesis of selenoproteins by overexpressing a mutant selenocysteine t-RNA caused glucose intolerance and diabetes-like phenotype in mice (Labunskyy et al. 2011).

Concerning ALS, it seems that the speciation of selenium (e.g., selenite or selenate vs organic forms) can determine the neurotoxicity of selenium in humans (Vinceti et al. 2019b). Accordingly, Vicenti and collaborators have recently demonstrated that the speciation of selenium in the cerebrospinal fluid of patients with mild cognitive deficits predicted the risk of progression to Alzheimer's disease, with selenate $\left(\mathrm{Se}^{+6}\right)$ increasing the risk significantly (Vinceti et al. 2017). Thus, in relation to cancer and other degenerative diseases, the role of selenium seems to have a $U$ inverted shape curve with a relatively narrow range of selenium for the optimum physiological effects (Fig. 1). Another point that is highly critical and little explored is the speciation of selenium as a

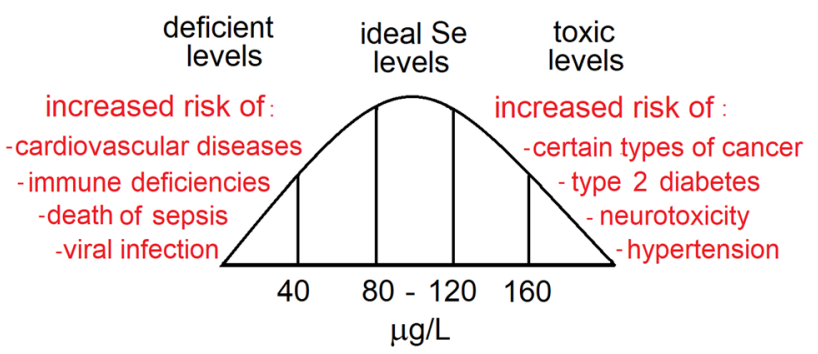

Fig. 1 U inverted shaped curve for selenium levels in humans. Low selenium status can increase the risk of immunological malfunctioning, cardiovascular diseases, sepsis severity, virus infection, and cognitive deficits. High levels of blood selenium can be associated with an increased risk of developing certain types of cancer (e.g., melanoma and prostate cancer), hypertension, type 2 diabetes, and neurodegenerative diseases (e.g., ALS and Alzheimer's Dementia). In the figure, the ideal levels of selenium were arbitrarily based on the optimal blood activity of glutathione peroxidase (see below). Selenium plays important physiological functions as a part of 25 selenoproteins in humans. At least one half of them are important oxireductases (e.g., 5 glutathione peroxidases (GPxs1-4 and 6); 3 thioredoxin reductases (TrxRs), which are involved in the regeneration of reduced thioredoxin (Trx); methionine sulfoxide reductase, which reduces oxidized methionine sulfoxide to methionine in proteins, 3 deiodinases (DIOs) that are involved in the metabolism of thyroid hormones (T3 and T4); selenophosphate synthetase and several selenoproteins without a clear-defined molecular role in cell physiology. The ideal physiological levels of selenium are not known, but for the blood GPx maximal activity, a level of selenium around $100 \mu \mathrm{g} \mathrm{L}^{-1}$ is required (Rea et al. 1979; Thomson et al. 1977, 1982). However, how blood GPx activity can predict the whole-body selenoproteins adequate physiological activity is unknown. There is also epidemiological evidence, suggesting that above $120 \mu \mathrm{g} \mathrm{L}^{-1}$, selenium can start to facilitate the installation of pathological conditions (Bastola et al. 2020) 
determinant of its toxic effects. Of particular toxicological importance, recent data have indicated that high levels of cationic selenium (e.g., selenate) in the cerebrospinal fluid of patients with mild cognitive impairment increases the risk of conversion from mild cognitive impairment to Alzheimer's Disease (Vinceti et al. 2017).

In this review, we will emphasize the potential pharmacology and toxicology of synthetic organoselenium compounds and some naturally occurring organoselenium amino acids (e.g., selenomethionine). The use of selenium as an important tool in organic synthesis and as a pharmacological agent goes back to the middle of the nineteenth and the beginning of the twentieth centuries. Notably, the rediscovery of ebselen (which was originally synthesized in 1924) and its investigation in several clinical trials in different types of human pathologies have motivated the search for new selenium-containing molecules with pharmacological properties (Masaki et al. 2016a; Ogawa et al. 1999; Saito et al. 1998; Singh et al. 2016; Yamaguchi et al. 1998). One point that has further stimulated the search for novel organoselenium compounds is the successive failures of ebselen or its low effectiveness as therapeutic agent (Beckman et al. 2016; Kil et al. 2017; Masaki et al. 2016a; Ogawa et al. 1999; Saito et al. 1998; Yamaguchi et al. 1998).

However, here, we have to emphasize that ebselen is still under clinical trials to treat bipolar disorder (Sharpley et al. 2020a) and has been registered for two clinical trials with moderate and severe COVID-19 patients (Haritha et al. 2020). Besides, ethaselen, an ebselen derivative, is in the recruiting phase of a clinical trial to treat lung cancer. The compound has been effective in a pre-clinical trial in human non-small cell lung cancer models (Ye et al. 2017; Zheng et al. 2017b,2019b). In short, though ebselen has not been approved to treat a specific disease, its safety in humans has been an indication that organoselenium compounds can be promising therapeutic agents.

\section{Physiological chemistry of selenium: selenium as component of selenoproteins}

The physiological chemistry of selenium in animals is played almost exclusively by the selenocysteinyl residue(s) found in a few types of selenoproteins. Selenocysteine is an analogue of cysteine and serine. The human genome codifies 25 selenoproteins that have usually only one residue of selenocysteine (Sec); the exception is the selenoprotein $\mathrm{P}$ that has near $10 \mathrm{~s}$ residues (for a brief description of selenoproteins function, see the legend of Fig. 1). The incorporation of selenium into the seryl-carbon skeleton is complex and occurs at the level of the transfer RNA (t-RNA[Ser]Sec) (in a process named co-translational incorporation of $\mathrm{Sec}$ in its t-RNA and then in the selenoproteins) (for reviews, see Hatfield et al. 2014; Labunskyy et al. 2014). This t-RNA[Ser]
Sec is first loaded with a seryl residue by the action of a seryl t-RNA synthetase and metabolized to phosphoseryl-tRNA[Ser]Sec by the enzyme seryl-t-RNA[Ser][Sec] kinase (PSTK). Then, selenophosphate donates, via the reaction catalyzed by the enzyme selenocysteine synthase (SepSecS), the selenium atom to form the selenol group in the place of the phosphorylated $\mathrm{OH}$ group of serine (Fig. 2). The incorporation of the selenium atom in an organic moiety requires four enzymatic steps, including the binding of serine to the t-RNA[Ser]Sec, the phosphorylation of loaded seryl residue, the synthesis of selenophosphate by the reaction of HSe(selenide) with ATP (a reaction catalyzed by the selonoenzyme selenophosphate synthetase), and the incorporation of selenophosphate in the place of the phosphorylated $\mathrm{OH}$ group of serine. For details about the incorporation of selenium in the serine skeleton, see the reviews (Hatfield et al. 2014; Labunskyy et al. 2014; Serrão et al. 2018).

The entire process requires several steps, protein factors, e.g., the Sec-t-RNA[Ser]Sec dedicated translation elongation factor (EFSec), SECIS-binding protein 2 (SBP2 or SECISBP2), ribosomal protein eL30, translation initiation factor 4A3 (eIF4A3), nucleolin, Secp43 or t-RNA selenocysteine 1-associated protein 1 (TRNAU1AP) and SepSecS), the specific RNA sequences (the selenocysteine insertion sequence or SECIS elements), and the t-RNA[Ser]Sec. The machinery utilized in the synthesis of selenoproteins interprets the stop codon UGA as selenocysteine only when it is present within the RNA sequences of the selenoproteins. The key players here are the SECIS elements, which in vertebrates are non-codifying RNA regions adjacent to the selenoprotein sequence, and selenocysteine-t-RNA (Bulteau and Chavatte 2015; Howard and Copeland 2019; Serrão et al. 2018; Simonović and Puppala 2018).

As briefly commented in the previous paragraph, the existence of these SECIS elements forming a stem-loopstem-loop structure with near 100 nucleotides in the 3 '-untranslated region of human 25 selenoprotein mRNAs is indispensable for the proper insertion of Sec in the selenoproteins. In fact, the stem-loop-stem-loop forming the SECIS bends or kinks itself toward the UGA codon inside the selenoprotein sequence and, inside of the ribosome, SECIS interacts with the t-RNA loaded with Sec and with protein factors described just above, allowing the release of the transporter and the incorporation of selenocysteine in the nascent polypeptide. For reviews about the molecular players in the noncanonical incorporation of Sec at UGA codon in selenoproteins (i.e., about the recoding of UGA), see (Bulteau and Chavatte 2015; Howard and Copeland 2019; Simonović and Puppala 2018). The selenocysteinyl residue, specifically its selenol group, is the softest of the nucleophile centers found in biomolecules. Accordingly, several selenoproteins are oxidoreductases enzymes, where the $-\mathrm{SeH}$ $\left(-\mathrm{Se}^{-}\right)$of the selenocysteine participates in the catalysis. 


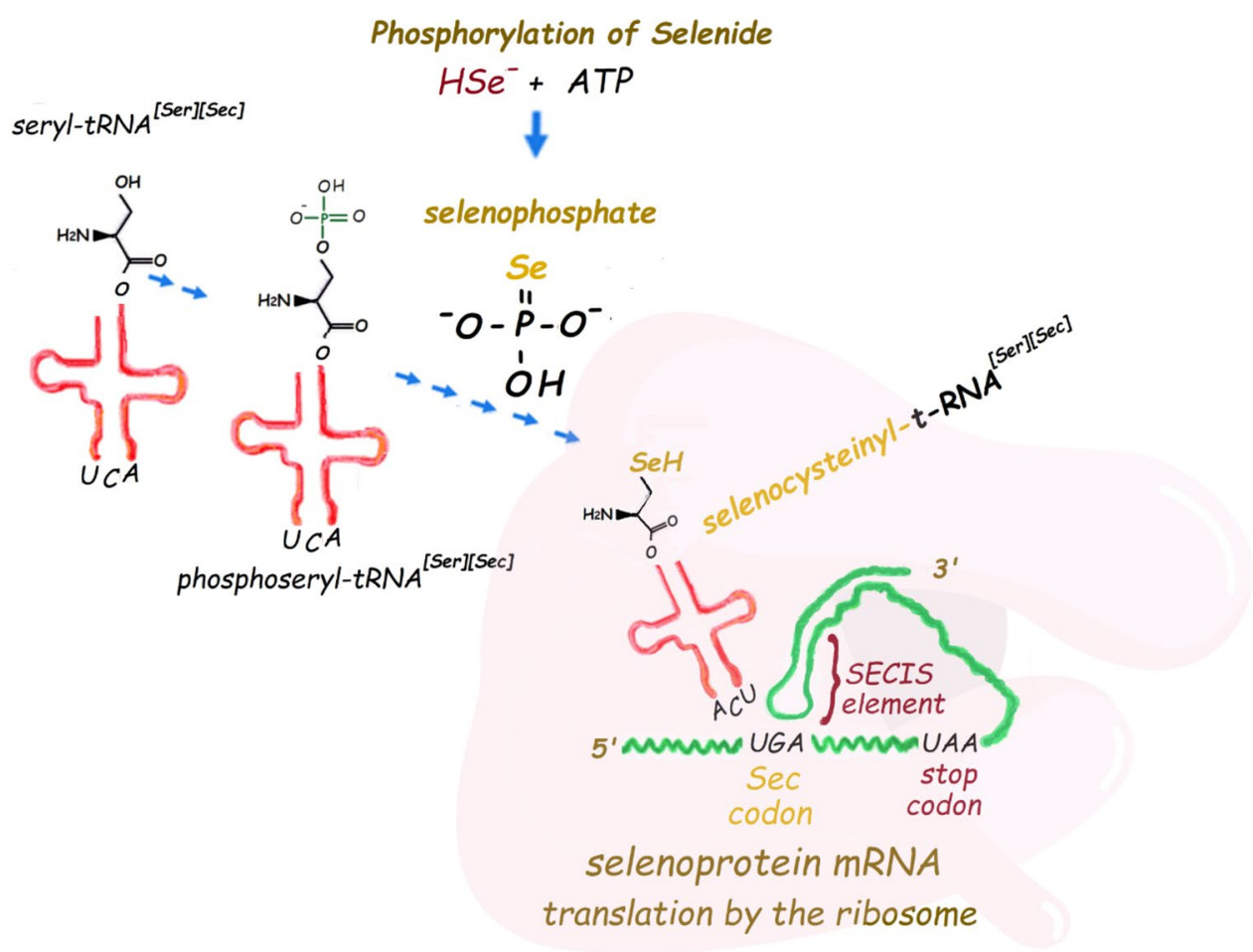

Fig. 2 Incorporation of selenide in the phosphoseryl-t-RNA[Ser]Sec and synthesis of selenocysteinyl-t-RNA[Ser]Sec after the reaction of selenophosphate with the phosphorylated hydroxyl group of serineloaded t-RNA[Ser]Sec. Selenocysteine is released from the t-RNA when the ribosome reads the UGA codon inside the mRNA sequence of a selenoprotein. The recoding of UGA codon to selenocysteine depends on the SECIS elements (which in mammals is a non-cod-

The well-characterized selenoproteins include glutathione peroxidase isoforms (e.g., GPx1 and GPx4); thioredoxin reductase isoforms (TrxR1, TrxR2, TrxR3), iodothyronine deiodinases (DIO1, DIO2, and DIO3), methionine sulfoxide reductase B (MrrB), selenophosphate synthetase, and selenoprotein $\mathrm{P}$. There are a group of endoplasmatic reticulumresident selenoproteins which the molecular roles are still elusive (Pitts and Hoffmann 2018; Addinsall et al. 2018; Gennadyevna 2020; Pitts and Hoffmann 2018). In addition to DIO2, selenoproteins $\mathrm{K}, \mathrm{M}, \mathrm{N}, \mathrm{S}, \mathrm{T}$, and selenoprotein $15 \mathrm{kDa}$ are found in the endoplasmic reticulum, where they appear to be involved in the regulation of $\mathrm{Ca}^{2+}$ levels, protein folding, inflammatory processes, and oxidative stress (Addinsall et al. 2018; Gennadyevna 2020; Pitts and Hoffmann 2018; Pothion et al. 2020; Shchedrina et al. 2010).

As commented above, the physiological chemistry of selenium seems to be played almost exclusively by the $-\mathrm{SeH}$ group of selenoproteins. Recently, it was demonstrated that selenocysteine can be incorporated in the uncoupling protein (UCP1) in the place of cysteine (Jedrychowski et al. 2020). The process is not a random incorporation of selenocysteine, but occurs in a specific cysteinyl residue (Cys253). ing mRNA forming a stem-loop structure that kinks to interact with the t-RNA). The translation of the in-frame UGA codons inside the genes of selenoproteins also requires several protein factors that are not indicated in the figure (for more details, consult the text or the reviews Bulteau and Chavatte 2015; Howard and Copeland 2019; Simonović and Puppala 2018)

The incorporation of selenocysteine is expected to occur via its t-RNA[Ser][Sec], because selenocysteine does not exist as a free amino acid in the presence of oxygen and water. However, the reasons why a cysteine codon can interact with the t-RNA[Ser][Sec] are unknown. Despite this, the data published by Jedrychowski and collaborators may open a new role for selenium as a modulator of the cysteine physiological function, via specific incorporation of selenocysteine in the place of specific cysteinyl residue in a small portion of synthesized thiol-containing proteins (for instance, for replacement of Cys 253 in UCP1, about $1.5 \%$ of this position was loaded with Sec) (Jedrychowski et al. 2020).

\section{Toxicity of organoselenium compounds}

\section{Interaction of selenium with thiols}

Herein, we will emphasize the biochemistry of interaction of organoselenium compounds with thiols from molecules of biological significance and their implications, without highlighting the pathophysiological processes associated 
with high levels of selenium. Among the organoselenium compounds, we will address primarily selenides and diselenides, such as ebselen, diphenyl diselenide, and some derivatives of them.

The molecular mechanisms involved in selenium toxicity are not completely elucidated; however, the interaction of inorganic and organic selenium with low- and high-molecular-weight thiols plays a central role in their toxicity. This evidence was first reported to inorganic forms, in which classical experiments showed the effectiveness of selenite in oxidizing sulfhydryl groups, producing disulfides and an unstable intermediary containing $-\mathrm{S}-\mathrm{Se}-\mathrm{S}-$ bonds (Ganther 1968; Painter 1941; Tsen and Tappel 1958). Afterward, studies demonstrated that the oxidation of thiols, such as glutathione (GSH) and cysteine, by selenite produced the radical superoxide (Seko et al. 1989; Spallholz 1994). To date, the oxidation of thiols has also been the basis to explain the toxicity of a variety of organoselenium compounds (Barbosa et al. 2017; Nogueira and Rocha 2011; Prigol et al. 2012) and mounting evidence has pointed out that reactive species (RS) formation contributes for the toxicity of many compounds (Nogueira and Rocha 2011; Prigol et al. 2012). In effect, the occurrence of oxidative stress and related phenomena has been highlighted in numerous in vitro and in vivo studies with organoselenium compounds. Interestingly, the increased production of RS accompanied by cell viability loss, DNA damage, and apoptosis are considered important pro-oxidant effects elicited by some organoselenium compounds against cancer cells, virus, and fungal pathogens (Álvarez-Pérez et al. 2018; Chen et al. 2020; Sies and Parnham 2020; Thangamani et al. 2017). It is important to mention here that the thiol oxidation may also subsidize the antioxidant effects of some selenium compounds via activation of antioxidant gene expression.

Therefore, the systematic study of oxidation of sulfhydryl groups from biological thiol-containing molecules by organoselenium compounds has facilitated the identification of potential "molecular targets" that might support both selenium pro-oxidative and antioxidant effects. In this context, we will cite here some in vitro and in silico (computational) studies toward specific interactions of some organoselenium compounds with proteins containing vicinal thiol groups, which are more efficiently oxidized than monothiol molecules.

\section{In vitro molecular toxicity of organoselenium compounds}

Although the molecular mechanisms involved in toxicity of organoselenium compounds are still not completely understood, the interaction with thiols is pointed out as a key phenomenon. Similar to inorganic selenium molecules, the interaction of the sulfur atom from thiols with the selenium atom from organoselenium compounds ( $\left.{ }^{\cdots \cdots} \cdot \mathrm{Se}\right)$ can lead to the formation of a selenenyl-sulfide bond ( $\mathrm{S}-\mathrm{Se}$ ), an adduct able to impair the activity of sulfhydryl enzymes. In fact, the toxic effects of several selenides and diselenides have been related to their potential in disrupting the activity of thiol-containing enzymes via oxidation of cysteinyl residues (Barbosa et al. 1998; Chaudiere et al. 1992; Galant et al. 2017, 2020; Nogueira and Rocha 2011; Quispe et al. 2019; Yu et al. 2017).

Focusing on diphenyl diselenide and its derivatives, the more precise findings include mainly those toward the enzyme $\delta$-aminolevulinate dehydratase ( $\delta$-ALA-D). $\delta$-ALA-D catalyzes the condensation of two molecules of 5-aminolevulinic acid to porphobilinogen, a monopyrrol precursor of prosthetic group heme, and due to its sulfhydryl nature, it has been commonly used in toxicological researches as an indicator of toxicity caused by pro-oxidant agents (Chaudiere et al. 1992; Ecker et al. 2018; Klimaczewski et al. 2018; Rocha et al. 2012c).

The active site of $\delta$-ALA-D possesses three cysteine residues coordinated with $\mathrm{Zn}^{2+}$, which prevent the formation of disulfide bridges between the sulfhydryl groups. The proximity of the cysteine groups in the active site renders to enzyme a high sensitivity to oxidation (Nogara et al. 2020; Rocha et al. 2012c; Saraiva et al. 2012).

The first studies demonstrating the diphenyl diselenide potential inhibitory on $\delta$-ALAD were carried out comparing the animal and plant enzymes (Barbosa et al. 1998; Farina et al. 2002). These findings revealed that diphenyl diselenide inhibited the activity from the animal enzyme, but not from the plant, in which the active site has aspartic acid instead of cysteine residues and the metal $\mathrm{Mg}^{2+}$ in the place of $\mathrm{Zn}^{2+}$. Since that, various other diphenyl diselenide derivatives were reported as inhibitors of the enzyme from different sources, as well (Nogueira and Rocha 2011; Nogueira et al. 2004; Rocha et al. 2012a, b, c).

In general, the mechanism by which diphenyl diselenide and its derivatives inhibit the $\delta$-ALA-D activity involves two steps of cysteine oxidation: (1) the first oxidation involves the attack of the selenium atom from diphenyl diselenide by the most reactive cysteinyl residue to yield the intermediate (E-S-SePh); and (2) the attack of the second more nucleophilic cysteinyl residue to the $\mathrm{S}-\mathrm{Se}-$ bond of the intermediate $(\mathrm{E}-\mathrm{S}-\mathrm{SePh})$, generating the oxidized enzyme $(\mathrm{E}-(\mathrm{S}-\mathrm{S}))$ and two molecules of selenol $(\mathrm{PhSeH})($ Scheme 2$)$ (Rocha et al. 2012c; Saraiva et al. 2012).

By docking analyses, the cysteine 124 residue from the active site of the enzyme was identified as the nucleophilic center that initiates the attack on the Se-Se bond from diselenides, forming an $\mathrm{E}-\mathrm{S}-\mathrm{SePh}$ as intermediate. The vicinal cysteine 132 residue was identified as responsible for the subsequent attack to the S-Se bond, resulting in the formation of the disulfide bond between cysteines 124 and 132 


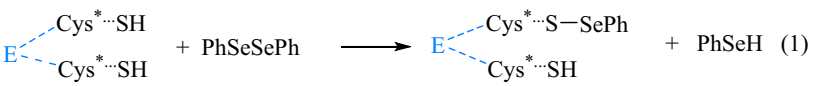

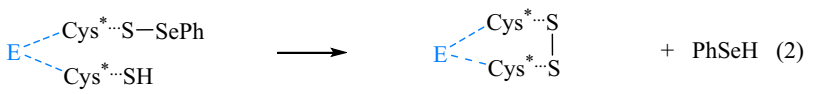

Scheme 2 Molecular mechanism of oxidation of $\delta$-ALAD catalytic thiols by diphenyl diselenide

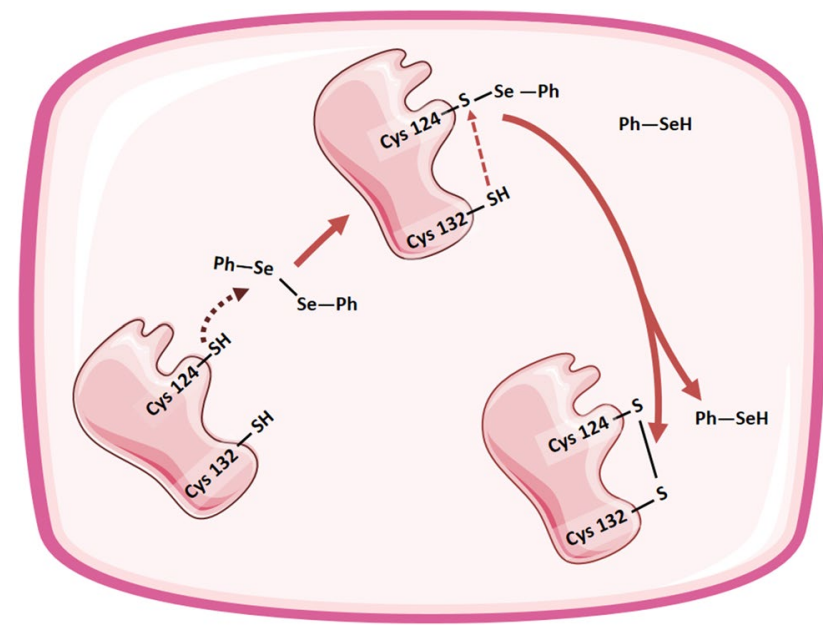

Fig. 3 Additional proposed molecular mechanism showing the oxidation of catalytic cysteine (cys) 124 and 132 from human $\delta$-ALAD by diphenyl diselenide, obtained by in silico studies (Nogara et al. 2020; Saraiva et al. 2012)

from $\delta$-ALA-D. Along with diphenyl diselenide, these interactions were also shown in silico for $p$-chloro, $p$-methoxy, and $m$-trifluoromethyl diselenide derivatives (Saraiva et al. 2012) (Fig. 3).

A very recent docking study was performed with sources of $\delta$-ALA-D enzyme from Homo sapiens (Hsס-AlaD), Drosophila melanogaster (Dmס-AlaD), and Cucumis sativus (Csס-AlaD), and the results corroborated the previous findings and provided more information about the mechanism of action (Nogara et al. 2020). Nogara and collaborators also reported the interaction of diphenyl diselenide with the cysteine residues from $\mathrm{Hs} \delta-\mathrm{AlaD}$ and $\mathrm{Dm} \delta-\mathrm{AlaD}$, but

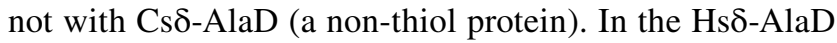
active site, they found that the selenium atoms of diphenyl diselenide interacted with the carboxylic group of aspartate 120 and the $\mathrm{Zn}^{2+}$ ion, besides the thiolate group from cysteine 124. In the Dmס-AlaD, selenium atoms interacted with arginine 205, proline 212, phenylalanine 204, tyrosine 20, and arginine 217 via $\mathrm{H}$ bond, and with the cysteine 122 . Interestingly, they found that the diphenyl diselenide putative metabolite, phenylseleninic acid, as well as other oxidized organoselenium forms presented similar binding pose, interacting with the cysteine 124 and 122 residues from the active site from $\mathrm{Hs} \delta-\mathrm{AlaD}$ and $\mathrm{Dm} \delta-\mathrm{AlaD}$, respectively (Nogara et al. 2020).

Because organoselenium compounds are highly prone to suffer a nucleophilic attack by cysteinyl residues, the activity of other sulfhydryl enzymes has been usually carried out to test the in vitro pro-oxidant potential of novel organochalcogens. Among them, the enzymes $\mathrm{Na}^{+}, \mathrm{K}^{+}$ATPase and lactate dehydrogenase (LDH) have been investigated (Chagas et al. 2013a; Kade et al. 2009; Lugokenski et al. 2011). In addition to diphenyl diselenide, herein, we highlighted the toxicological studies showing the inhibitory effects of ebselen, 4-(4-fluorophenylseleno)-3-phenylisoquinoline), chloro (4-(4-chlorophenylseleno)-3-phenylisoquinoline), trifluoro (4-(3-trifluoromethylphenylseleno)-3-phenylisoquinoline), and bis(phenylimidazoselenazolyl) diselenide on $\delta$-ALA-D and $\mathrm{Na}^{+}, \mathrm{K}^{+}$ATPase activities from rat tissues (Chagas et al. 2015; Sampaio et al. 2017a).

Recently, the activities of both enzymes were also used for screening the toxicity of novel zidovudine (AZT)-based selenides on human erythrocytes. Among 5'-phenylseleno-, $p$-chloro-, $p$-methyl-AZT derivatives, the $p$-methyl substituted AZT-derivative was the least toxic and did not cause $\delta$-ALA-D and $\mathrm{Na}^{+}, \mathrm{K}^{+}$ATPase inhibition, thiol depletion, and eryptosis; whereas the $5^{\prime}$-phenylseleno- and $p$-chloroderivatives inhibited both $\delta$-ALA-D and $\mathrm{Na}^{+}, \mathrm{K}^{+}$ATPase activities, causing thiol depletion, and eryptosis (Ecker et al. 2018).

Regarding the oxidation of thiols from low-molecularweight compounds, increasing evidence indicates that the oxidation rate seems to be dependent on $\mathrm{pH}$ and independent of thiol group pKa. At pH 7.4, cysteine and dithiothreitol were more reactive with diphenyl diselenide, whereas 2,3-dimercapto-1-propanesulfonic acid, GSH, and dimercaptosuccinic acid exhibited a low reactivity (Hassan and Rocha 2012).

\section{Organoselenium compounds: thiol depletion, reactive species overproduction, and mitochondrial dysfunction}

As mentioned before, some organoselenium compounds may exhibit strong electrophilic activity, forming selenenylsulfide bonds with the cysteinyl residues from non-protein and protein thiols. Therefore, the activity of several protein families, including antioxidant enzymes as well as the GSH cell levels can be affected, and represent one of the main mechanisms by which organoselenium compounds modulate a wide spectrum of related biological processes. Among them, we highlighted herein those associated with oxidative stress, in which thiol depletion, RS overproduction, DNA damage, and mitochondrial dysfunctions are usually pointed out as key end-points. 
Although ebselen and diphenyl diselenide are well recognized as antioxidant active agents, both compounds may exacerbate the production of RS at relatively high concentrations in vitro. In a range from 10 to $50 \mu \mathrm{M}$, ebselen and diphenyl diselenide induced RS overproduction, accompanied by loss of viability, and DNA damage in human white cells (Bueno et al. 2018; Caeran Bueno et al. 2013). Similarly, ebselen caused an increase in RS levels, viability loss, -SH oxidation, and calcium dyshomeostasis in cultured astrocytes (Santofimia-Castano et al. 2013, 2016).

It is important to note that depletion of thiols accompanied by the increase in RS production has been suggested as possible mechanisms involved in the action of ebselen, diphenyl diselenide, and its derivatives against a diversity of fungal pathogens (Bueno Rosseti et al. 2014; Jaromin et al. 2018; $\mathrm{Ngo}$ et al. 2016; Rosseti et al. 2015; Thangamani et al. 2017).

In line with this, a study toward Saccharomyces cerevisiae showed that the deleterious effects of diphenyl diselenide on growth, size, and membrane permeability were followed by a marked RS overproduction at the highest concentration tested $(10 \mu \mathrm{M})$ (Galant et al. 2017). Diphenyl diselenide and its dicholesteroyl diselenide derivative were genotoxic and mutagenic to S. cerevisiae, and these effects were associated with oxidative damage, because $\mathrm{N}$-acetylcysteine partially reversed the toxicity of these compounds (de Oliveira et al. 2014).

Toxicological studies have indicated that mitochondria are potential targets for pro-oxidant action of selenides and diselenides, including ebselen and diphenyl diselenide. In general, the deleterious effects are demonstrated at concentrations ranging from 10 to $100 \mu \mathrm{M}$. One of the first studies that simultaneously evaluated the effect of ebselen and diphenyl diselenide on mitochondria demonstrated that both compounds caused mitochondrial depolarization and swelling, effects that were associated with thiol oxidation, given that dithiotreitol prevented them (Puntel et al. 2010). In an extension of this study, using renal and hepatic mitochondria, ebselen and diphenyl diselenide inhibited the activity of complex I and II, without changing the complex III and IV. These effects were reversed by GSH and then related to the oxidation of critical thiol groups from mitochondrial complexes I and II (Puntel et al. 2013).

In the rat liver mitochondria, a recent study investigated diphenyl diselenide derivatives containing $o$-methoxy and $p$-methyl groups substituted in the aryl $(25-100 \mu \mathrm{M})$ and revealed that only the compound containing the $p$-methyl group affected the mitochondrial membrane potential and decreased the State III respiration from $25 \mu \mathrm{M}$ (Stefanello et al. 2020). Mitochondria from rat hippocampal astrocytes exposed to ebselen at a concentration of $100 \mu \mathrm{M}$ showed disturbances in the membrane potential and calcium levels along with RS overproduction (Santofimia-Castano et al. 2013).

As the mitochondria are highly sensitive to redox status, pro-oxidant agents can disrupt mitochondrial homeostasis and trigger cell death via apoptosis. As stated above, diphenyl diselenide and ebselen induced in vitro deleterious effects on mitochondria that could elicit apoptosis. However, some in vitro findings show that exposure of healthy cells to both compounds did not culminate with apoptosis. In fact, human leukocytes exposed to diphenyl diselenide and ebselen, ranging from 10 to $50 \mu \mathrm{M}$, presented changes in mRNA expression of antioxidant enzymes, such as catalase, superoxide dismutase, and glutathione peroxidase, and increase in RS production, but did not undergo apoptosis (Caeran Bueno et al. 2013). Accordingly, exposure of rat hippocampal astrocytes to ebselen $(10-100 \mu \mathrm{M})$ caused viability loss, endoplasmic reticulum, and mitochondrial stress, without changing the activity of caspase-3, an apoptosis activation marker (Santofimia-Castaño et al. 2016; Santofimia-Castano et al. 2013).

On the other hand, the activation of death signaling by many organoselenium compounds toward unhealthy cells and pathogens has driven studies on the synthesis and screening for anti-cancer, anti-viral, and anti-fungal applications (these will be further addressed in detail at the pharmacological section).

\section{In vivo toxicity of organoselenium compounds}

Although diphenyl diselenide and ebselen are recognized as compounds with low toxicity in vivo, at high doses, they can elicit toxic effects, which vary a lot according to the species, exposure time, and route of administration. As demonstrated in vitro, mechanistically, the in vivo toxicity of ebselen, diphenyl diselenide, and its derivatives has been associated with oxidative events, including thiol depletion, lipid peroxidation, and inhibition of sulfhydryl enzymes, such as $\delta$-ALA-D, $\mathrm{Na}^{+}, \mathrm{K}^{+}$ATPase, and LDH (Nogueira and Rocha 2011; Nogueira et al. 2004). To date, the toxicological implications from acute and chronic exposures to organoselenium compounds on mammalian models have not been extensively reported in the literature. Therefore, we will also include here some findings found in non-mammalian models. Table 1 summarizes the acute and chronic effects of some organoselenium compounds.

\section{Acute exposure}

In rodents, the toxicity of diphenyl diselenide and ebselen varies depending on the route of administration, age, and species (Table 1). After acute administration by the intraperitoneal (i.p) route, diphenyl diselenide was more toxic to mice than rats (Meotti et al. 2003). On the other hand, the 
Table 1 Effects of acute and chronic treatments with diphenyl diselenide, ebselen, and selenomethionine

\begin{tabular}{|c|c|c|c|c|}
\hline \multicolumn{3}{|c|}{ Acute exposure } & \multicolumn{2}{|l|}{ Chronic exposure } \\
\hline Species & Treatments & Effects & Treatments & Effects \\
\hline \multirow[t]{8}{*}{ Mice } & Diphenyl diselenide & & & \\
\hline & $210 \mu \mathrm{mol} \mathrm{kg}{ }^{-1}(\text { i.p })^{\mathrm{a}}$ & Mortality & & \\
\hline & $150 \mu \mathrm{mol} . \mathrm{kg}^{-1}$ (i.p) & $\uparrow$ PTZ-induced seizure & & \\
\hline & $10 \mathrm{mg} \mathrm{kg}^{-1}$ (i.p) & Stereotypy & & \\
\hline & Ebselen & & & \\
\hline & $340 \mu \mathrm{mol} \mathrm{kg}{ }^{-1}(\text { i.p })^{\mathrm{a}}$ & Mortality & & \\
\hline & Selenomethionine & & Selenomethionine & \\
\hline & $\begin{array}{l}\sim 8 \mathrm{mg} \mathrm{kg}^{-1} \text { (i.p) } \\
8 \mathrm{mg} \mathrm{Se} \mathrm{kg}^{-1} \text { (i.p) }\end{array}$ & $\begin{array}{l}\text { Mortality } \\
\text { Hepatic lipid peroxidation }\end{array}$ & $\begin{array}{l}0.2 \text { and } 2 \mathrm{mg} \mathrm{g}^{-1}(\mathrm{p} . \mathrm{o}) \\
50 \text { days }\end{array}$ & $\begin{array}{l}\uparrow \text { Liver } \\
\text { Lipoperoxidation } \\
\text { Arsenic-induced }\end{array}$ \\
\hline \multirow[t]{10}{*}{ Rats } & Diphenyl diselenide & & Diphenyl diselenide & \\
\hline & $\begin{array}{l}1200 \mu \mathrm{mol} \mathrm{kg}^{-1} \text { (i.p) } \\
10 \mathrm{mg} \mathrm{kg}^{-1} \text { (i.p) } \\
50 \text { to } 500 \mathrm{mg} \mathrm{kg}^{-1} \text { (i.g) }\end{array}$ & $\begin{array}{l}\text { Mortality } \\
\text { Anxiety } \\
\text { Seizures, brain lipid peroxidation in pups }\end{array}$ & $\begin{array}{l}1 \mathrm{mg} \mathrm{kg}^{-1} \text { (i.p) } \\
21 \text { days } \\
\text { Ebselen }\end{array}$ & $\begin{array}{l}\uparrow \mathrm{Hg} \text { deposition } \\
\text { In liver and brain }\end{array}$ \\
\hline & & & $\begin{array}{l}10 \mathrm{mg} \mathrm{kg}^{-1} \text { (s.c) } \\
21 \text { days }\end{array}$ & $\begin{array}{l}\text { Hepatic lipoperoxidation } \\
\text { In suckling }\end{array}$ \\
\hline & & & Selenomethionine & \\
\hline & & & $\begin{array}{l}1.2-1.8 \mathrm{mg} \mathrm{kg}^{-1} \\
\text { (p.o) } \\
13 \text { weeks }\end{array}$ & $\begin{array}{l}\downarrow \text { Weight } \\
\text { Liver and } \\
\text { pancreas damage }\end{array}$ \\
\hline & Ebselen & & & \\
\hline & $400 \mu \mathrm{mol} \mathrm{kg}{ }^{-1}(\mathrm{i} . \mathrm{p})^{\mathrm{a}}$ & Mortality & & \\
\hline & Selenomethionine & & & \\
\hline & $\sim 4 \mathrm{mg} \mathrm{kg}^{-1}(\mathrm{i} . \mathrm{p})^{\mathrm{a}}$ & Mortality & & \\
\hline & $1 \mathrm{mg} \mathrm{kg}^{-1}$ (i.p) & Pancreatic damage & & \\
\hline \multirow[t]{2}{*}{ Rabbits } & Diphenyl diselenide & & & \\
\hline & $500 \mathrm{mmol} \mathrm{kg}^{-1}$ (i.p) & $\begin{array}{l}\text { Mortality, hepatoxicity, and brain oxidative } \\
\text { stress }\end{array}$ & & \\
\hline \multirow[t]{3}{*}{ Fly $^{\mathrm{b}}$} & & & Diphenyl diselenide & \\
\hline & & & $\begin{array}{l}0.5-2 \mu \mathrm{M}(\mathrm{p} . \mathrm{o}) \\
10 \text { days }\end{array}$ & Developmental delay \\
\hline & & & $\begin{array}{l}1-10 \mu \mathrm{M}(\mathrm{p} . \mathrm{o}) \\
3 \text { days }\end{array}$ & $\uparrow \mathrm{Hg}$ toxicity in adult \\
\hline \multirow[t]{7}{*}{ Fish } & & & Diphenyl diselenide & \\
\hline & & & $\begin{array}{l}5 \text { ppm (p.o) } \\
60 \text { days }\end{array}$ & $\begin{array}{l}\downarrow \text { weight } \\
\text { Oxidative damage } \\
\text { Silver catfish and Cyprinius carpo }\end{array}$ \\
\hline & & & Selenomethionine & \\
\hline & & & $\begin{array}{l}10-30 \mu \mathrm{g} \mathrm{g}^{-1}(\mathrm{p} . \mathrm{o}) \\
90 \text { days }\end{array}$ & $\begin{array}{l}\text { Behavioral changes, cardiac dysfunctions } \\
\text { In adult Zebrafish }\end{array}$ \\
\hline & & & $\begin{array}{l}30-60 \mu \mathrm{g} \mathrm{g}^{-1}(\mathrm{p} . \mathrm{o}) \\
30 \text { days }\end{array}$ & $\begin{array}{l}\text { Cognitive impairment, brain oxidative stress } \\
\text { In adult Zebrafish }\end{array}$ \\
\hline & & & $\begin{array}{l}100 \mu \mathrm{g} \mathrm{L}^{-1} \text { (p.o) } \\
48 \mathrm{hpf}\end{array}$ & $\begin{array}{l}\text { Embryonic teratogenesis } \\
\text { in Zebrafish }\end{array}$ \\
\hline & & & $\begin{array}{l}30-60 \mu \mathrm{g} \mathrm{g}^{-1} \text { (p.o) } \\
30 \text { days }\end{array}$ & $\begin{array}{l}\text { Growth retard, mortality, hematological } \\
\text { disturbances in } \\
\text { juvenile Steelhead trout }\end{array}$ \\
\hline \multirow[t]{2}{*}{ Lambs } & Selenomethionine & & & \\
\hline & $1-8$ mg/kg (p.o) & $\begin{array}{l}\text { Tachypnea, myocardial } \\
\text { necrosis, pulmonary edema }\end{array}$ & & \\
\hline
\end{tabular}

${ }^{\mathrm{a}} \mathrm{LD}_{50}$ the dose of a test substance that is lethal for $50 \%$ of the animals, $h p f$ hours post-fertilization, PTZ pentylenetetrazol, Fly ${ }^{b}$ Drosophila melanogaster 
i.p. administration of diphenyl diselenide-loaded nanocapsules $\left(50-1000 \mu \mathrm{mol} \mathrm{kg}{ }^{-1}\right.$ ) did not cause overt toxicity or death in mice (Stefanello et al. 2015a). The $\mathrm{LD}_{50}$ values for ebselen were very similar when intraperitoneally injected in mice and rats (Meotti et al. 2003).

Diphenyl diselenide was also toxic to rabbits, when administered by the i.p route at a dose of $500 \mu \mathrm{mol} \mathrm{kg}-1$ caused mortality, hepatoxicity, and disruption of brain redox status (Straliotto et al. 2010).

By subcutaneous (s.c) and intragastric (i.g) routes, diphenyl diselenide usually exhibited lower toxicity than that observed after i.p administration. Acute intragastric or subcutaneous administration of diphenyl diselenide did not cause overt toxicity or death in rats and mice (da Luz Fiuza et al. 2015; Meinerz et al. 2014; Meotti et al. 2003; Wilhelm et al. 2009b).

By the intravenous route, sheep treated with a single dose of diphenyl diselenide $\left(6 \mu \mathrm{mol} \mathrm{kg}{ }^{-1}\right)$ did not have any overt sign of toxicity until the end of observational period, namely 37 days (Leal et al. 2018).

Especially toward central symptoms from acute treatments, the first investigations demonstrated that an i.p administration of diphenyl diselenide increased the pentilentetrazole-induced seizure in mice (Table 1), but not in rats (Brito et al. 2006). Regarding the age of animals, diphenyl diselenide administered by s.c or i.g route did not cause seizures in adult rats or mice; however, in 12-day-old rat pups, oral acute treatment induced seizure episodes (Table 1) (Prigol et al. 2007). Diphenyl diselenide acutely administered at the dose of $10 \mathrm{mg} \mathrm{kg}^{-1}$ induced stereotypy in mice and anxiety-like behavior in rats, manifestations that were related to the inhibition of the brain monoamino oxidase (MAO-B) activity and increased levels of pro-inflammatory marker tumor necrosis factor $\alpha$ (TNF), respectively (Figueira et al. 2015; Yamakawa et al. 2020).

In non-mammalian models, some findings showed that the acute treatment of zebrafish with diphenyl diselenide and diphenyl diselenide-loaded nanocapsules, at concentrations ranging from 0.1 to $2 \mu \mathrm{M}$, did not cause behavioral impairments and/or oxidative stress (Ferreira et al. 2019b; Ibrahim et al. 2014b).

\section{Chronic exposure}

The toxic effects elicited by diphenyl diselenide from chronic treatments also vary in relation to the organisms (Table 1). Most of chronic protocols have applied dietary diphenyl diselenide, which have revealed that the longterm intake is relatively safe for several species. Evidence has been found to suggest that dietary diphenyl diselenide, from 1 to $3 \mathrm{ppm}$, was relatively secure for rats, rabbits, and some fish species after months of exposure, without eliciting either systemic or central signals of toxicity (Baldissera et al. 2020a; Barbosa et al. 2008; de Bem et al. 2006; dos Santos et al. 2020). However, the intake of high concentrations for a long time may culminate with toxic effects in fish. In fact, Silver catfish and Cyprinius carpio fed with 5 ppm diphenyl diselenide, for 60 days, had reduction in the weight and body length, and also showed increased lipid peroxidation in the liver, brain, and muscle (Menezes et al. 2014, 2016).

Here, we highlight that diphenyl diselenide chronic treatment (Table 1) did not induce toxicity in rats, but enhanced the $\mathrm{Hg}$ accumulation in the liver and brain as well as potentiated motor deficits and body-weight loss caused by methylmercury (MeHg) (Dalla Corte et al. 2013).

In vivo chronic toxicity data with ebselen are scarcer than diphenyl diselenide, but there is evidence that chronic subcutaneous administration of the compound $(10 \mathrm{mg} / \mathrm{kg}$, for 21 days) to suckling rats culminated with lipid peroxidation and non-protein thiol depletion in the liver (Farina et al. 2004).

In invertebrates, literature brings some findings from $D$. melanogaster as an organism model to study toxicology and pharmacology of dietary diphenyl diselenide. Accordingly, the toxicity of dietary diphenyl diselenide was dependent on sex of $D$. melanogaster both in relation to total body thiol depletion and disruption in the mRNA expression of the antioxidant enzymes like catalase, superoxide dismutase, and glutathione $S$ transferase (Occai et al. 2018).

Leão and collaborators exposed flies to diphenyl diselenide during both developmental and adult phases. Dietary diphenyl diselenide, ranging from 0.5 to $2 \mu \mathrm{M}$, affected the normal developmental success of $D$. melanogaster and also enhanced the toxicity of $\mathrm{MeHg}$ on development. In adult flies, diphenyl diselenide ( 1 to $10 \mu \mathrm{M}$ ) did not induce toxic effects, but increased the toxicity of MeHg on climbing ability and survival of individuals (Leão et al. 2018).

Moreover, diphenyl diselenide and $\mathrm{MeHg}$ co-exposure increased the $\mathrm{Hg}$ levels in the flies, an effect that was related to the formation of a less excretable complex between the selenium from the organoselenium and $\mathrm{Hg}$. Accordingly, the same group had already demonstrated that diphenyl diselenide and $\mathrm{MeHg}$ co-exposure had increased the $\mathrm{Hg}$ content in the brain and liver of rodents (Dalla Corte et al. 2016, 2013). In flies and rats, the reduced intermediate of diphenyl diselenide (phenylselenol or selenophenol) may have reacted with $\mathrm{MeHg}$ to form a $\mathrm{PhSe}-\mathrm{HgMe}$ complex. This complex possibly facilitated the break of $-\mathrm{C}-\mathrm{Se}-$ and $-\mathrm{Hg}-\mathrm{C}-$ bonds, releasing the insoluble $\mathrm{HgSe}$ salt (Madabeni et al. 2020).

In vivo toxicity of diphenyl diselenide derivatives and other organoselenium compounds Similar to diphenyl diselenide, the toxic profile of diselenide derivatives varies depending on the species and administration route, and, in general, the intragastric and subcutaneous administrations were reported to be safer than intraperitoneal. For mice, the intragastric 
$\mathrm{LD}_{50}$ for diselenide derivatives, $p$-chloro and $p$-methoxyl substituted diselenides, was found to be $>1 \mathrm{mmol} \mathrm{kg}^{-1}$. For $m$-trifluoromethyl and benzylamino derivatives, the $\mathrm{LD}_{50}$ was estimated to be $>0.62$ and $350 \mathrm{mg} \mathrm{kg}^{-1}$, respectively (Ibrahim et al. 2019; Savegnago et al. 2009).

The intragastric administration of $p$-methoxyl-substituted diselenide-nanoencapsulated ( $25 \mathrm{mg} \mathrm{kg}^{-1}, 7$ days) did not cause any alteration in hematological and oxidative damage markers, and enhanced the selenium levels in the blood, kidney, and liver of mice (Sari et al. 2017).

By the subcutaneous route, $p$-chloro diselenide derivative $\left(1000 \mu \mathrm{mol} \mathrm{kg}^{-1}\right)$ did not induce overt sign of toxicity in mice and reduced the toxicity of $\mathrm{HgCl}_{2}$ on the liver and kidney, as indicated by the restoration of $\delta$-ALA-D, $\mathrm{Na}^{+}, \mathrm{K}^{+}$ ATPase, and lipid peroxidation to normal levels (de Freitas et al. 2012).

In rats, 2,2'-dithienyl diselenide derivative, at doses of 50 and $100 \mathrm{mg} \mathrm{kg}^{-1}$, caused systemic toxicity after intragastric administration, such as weight body loss, hepatotoxicity, inhibition of $\delta$-ALA-D activity, lipid peroxidation, and death (Chagas et al. 2013a).

Acute intraperitoneal administration of vinyl chalcogenide 3-methyl-1-phenyl-2-(phenylseleno)oct-2-en-1-one, at doses of $125-500 \mu \mathrm{g} \mathrm{kg}^{-1}$, was toxic to rats, increasing plasma alanine aminotransferase and causing hematological and behavioral changes. The compound also increased lipid and protein oxidation in the brain (de Andrade et al. 2014; dos Santos et al. 2012). Likewise, rats exposed chronically to this vinyl chalcogenide (i.p., 30 days), in addition to cause behavioral alterations, increased brain lipid and protein oxidation, thiol depletion, liver, and renal damage (Medeiros et al. 2012; Mello et al. 2012).

3,3'-Diselenodipropionic acid (DSePA), a synthetic derivative of selenocystine, has been extensively investigated as an antioxidant and radioprotective agent. The acute oral toxicity of DSePA is low in mice $\left(\mathrm{LD}_{50} \sim 200 \mathrm{mg} \mathrm{kg}^{-1}\right)$ and rat $\left(\mathrm{LD}_{50} \sim 25 \mathrm{mg} \mathrm{kg}^{-1}\right)$ when compared with its parent compound selenocystine and other organoselenium commonly used as a nutritional supplement, including methylselenocysteine and selenomethionine (Kunwar et al. 2018, 2020; Yang and Jia 2014). The mechanisms involved in the toxicity of DSePA have not been studied in detail, but considering its structure and its $\mathrm{LD}_{50}$ for rodents (which is greater than selenocystine); we can predict that its toxicity will be mediated by oxidation of thiol groups of critical target proteins. Although the acute in vivo toxicity of DSePA in mice has been described to be low in relation to selenocystine, we have to bear in mind the extreme sensitivity of humans to the toxic effects of selenocystine (Weisberger and Suhrland 1956). This aspect is important in view of the structural similarity of DSePA with selenocystine.

Regarding the in vivo toxicity of naturally occurring organoselenium compounds, we included here some toxicological studies published in the last decade with selenomethionine, methylselenocysteine, and selenocysteine, in which the findings with selenomethionine were the most prevalent (Table 1). Selenomethionine can be metabolized to selenide and provided selenium to be incorporated into selenoproteins. However, depending on the species, selenomethionine levels exceeding $0.2 \mathrm{ppm}$ can become toxic (Schrauzer 2003).

Human cases of acute and/or chronic selenomethionine poisoning are rare, but, recently, a fatal case of occupational acute intoxication with this powdered amino acid was reported. After trying to open a sealed bag-container, the L-selenomethionine powder blew out back onto 30 years-old man. Selenomethionine contaminated his skin and clothes, and was also inhaled. A few hours after the contamination (about $5 \mathrm{~h}$ ), the man died, and just before dying, he had abnormal blood $\mathrm{pH}$ (7.01), oxygen saturation (75\%), glucose $\left(17 \mathrm{mg} 100 \mathrm{~mL}^{-1}\right)$, bicarbonate $\left(12 \mathrm{mEq} \mathrm{L}{ }^{-1}\right)$, urea $\left(14 \mathrm{mg} \mathrm{dL}^{-1}\right)$, creatinine $\left(1.51 \mathrm{mg} 100 \mathrm{~mL}^{-1}\right), 11 \mathrm{mg} \mathrm{L}^{-1}$ of selenium (normal levels below $0.16 \mathrm{mg} . \mathrm{L}^{-1}$ ), and urine selenium levels of $25 \mathrm{mg} \mathrm{L}^{-1}$ (normal levels below $0.2 \mathrm{mg} \mathrm{L}^{-1}$ ) (Spiller et al. 2020).

In an experimental animal model, lambs orally administered with a single dose of selenium as selenomethionine (ranging from $\sim 1$ to $8 \mathrm{mg}$ of $\mathrm{Se} \mathrm{kg}^{-1}$ ) developed tachypnea, whose severity and time to recovery were dose-dependent. Histopathologic alterations were also observed in the animals exposed to the higher doses, including myocardial necrosis, pulmonary edema, and hemorrhage (Tiwary et al. 2006).

In rats, the i.p. $\mathrm{LD}_{50}$ of selenomethionine was estimated to be $\sim 4 \mathrm{mg} \mathrm{Se} \mathrm{kg}^{-1}$, whereas for mice $\sim 8 \mathrm{mg} \mathrm{Se} \mathrm{kg}^{-1}$ (Schrauzer 2003). Indeed, rats intravenously injected, at a bolus dose of $1.0 \mathrm{mg} \mathrm{Se} \mathrm{kg}^{-1}$ as selenomethionine, accumulated selenium preferably in the pancreas and had a significant increase in the serum amylase levels, a key marker of pancreatic damage.

The chronic toxicity of selenomethionine is considered lower than inorganic selenium forms, and often is reported in animals fed with too high levels. Rats fed for 8 weeks with selenomethionine (16 ppm of Se) did not develop signs of toxicity, whereas the same amount of selenium as sodium selenite produced hepatotoxicity, cardiotoxicity, and splenomegaly (Schrauzer 2003).

Oral administration of 0.5 and $1.0 \mathrm{mg}$ selenomethionine, for 13 weeks, did not also induce toxic effects in rats. However, higher concentrations (1.2 and $1.8 \mathrm{mg} \mathrm{Se} \mathrm{kg}^{-1}$ body weight.day ${ }^{-1}$ ) caused weight loss, liver, and pancreas damage and decreased food consumption (Schrauzer 2003).

In a mouse model, the supplementation of sufficient and excess levels of selenomethionine $\left(0.2\right.$ and $2 \mathrm{mg} \mathrm{Se}^{-1} \mathrm{~kg}^{-1}$, respectively), for 50 days, improved the basal immunological parameters impaired by arsenic intoxication, but the 
two doses increased the hepatic lipid peroxidation arsenicinduced (Rodríguez-Sosa et al. 2013).

In non-mammalian models, most of the literature about the toxicity of selenomethionine intake is in fish. In zebrafish, chronic exposure to elevated dietary selenomethionine (from 10 to $30 \mu \mathrm{g} \mathrm{g}^{-1}$ ) has been associated with impairments in behavioral performance, aerobic metabolic capacity, and energy homeostasis (McPhee and Janz 2014). These adverse effects were correlated with the negative impact of chronic dietary selenomethionine on cardiac function, because zebrafish exposed to similar concentrations had a marked decrease in the ventricular contractile rate, stroke volume, and cardiac output, as well as disruption in the mRNA expression of cardiac remodeling enzymes (Pettem et al. 2017).

Adult zebrafish exposed to selenomethionine $\sim 30$ and $60 \mathrm{\mu g} \mathrm{g}^{-1}$ of diet developed learning impairment, which was associated with oxidative stress and altered brain mRNA expression of dopaminergic system components (Naderi et al. 2017).

Chronic exposure of adult zebrafish to selenomethionine (34.1 $\mathrm{\mu g} \mathrm{g}^{-1}$; 90 days) also displayed changes in social learning via dysregulation of key genes of the serotonergic pathway (Attaran et al. 2020).

In zebrafish embryos, selenomethionine at $100 \mu \mathrm{g} \mathrm{L}^{-1}$ induced significant deformities (lordosis and craniofacial malformation), which were partially attributed to oxidative stress, since $\mathrm{N}$-acetylcysteine reduced the teratogenic signals (Arnold et al. 2016).

A study performed with steelhead trout in the juvenile stage fed on $\sim 8,15,30$, and $60 \mu \mathrm{g} \mathrm{Se} \mathrm{g}^{-1}$ diet in the form of selenomethionine, for 4 weeks, revealed that Se accumulated in a dose-dependent manner in all tissues. Moreover, the diets with selenomethionine at $30 \mu \mathrm{g} \mathrm{g}^{-1}$ or higher arrested growth and increased mortality and hematological disturbances (Lee et al. 2019).

\section{Pharmacology of organoselenium compounds}

The coronavirus COVID-19 pandemic, caused by the worldwide spread of new SARS-CoV-2, has dominated the work of researchers in an unprecedented global effort to lead to the rapid discovery of drugs with the clinical potential to fighting this new infectious disease for which no specific drugs or vaccines are available (Roser et al. 2020).

Since the nutritional essentiality of selenium as a trace element for human health has been demonstrated (Schwarz and Foltz 1957), the multifaceted aspects of this nutrient have attracted worldwide clinical and research interest (Allingstrup and Afshari 2015; Navarro-Alarcon and Cabrera-Vique 2008; Oldfield 1987; Rayman 2012).
However, the selenium status should be analyzed considering its U-shaped effects, exhibiting advantages in seleniumdeficient individuals but specific health risks in those with selenium excess (Duntas and Benvenga 2015; Misu et al. 2012; Rayman 2020; Rayman et al. 2012; Rayman and Stranges 2013; Rocourt and Cheng 2013; Zhou et al. 2013).

Particularly, in China, where COVID-19 emerged, the concentration of selenium in the soil, which generally reflects its presence in food and the selenium levels in human populations, varies from deficiency to excess (Dinh et al. 2018). Based on this premise and knowing the immunomodulatory property of selenium (Guillin et al. 2019; Steinbrenner et al. 2015), a very recent published study from Rayman group reported the better recovering of COVID-19 patients related to certain regions of China that had the most selenium in soil (Zhang et al. 2020c).

Selenium, as a cyclic selenyl amide ebselen, also emerges in the pandemic scenario as a potential repurposing approved pharmaceutical drug with anti-viral activity for the treatment of COVID-19 (Sies and Parnham 2020).

Considering what was mentioned before, in this chapter, the pharmacology of organoselenium compounds is discussed, emphasizing properties beyond their well-known antioxidant activity.

\section{Anti-viral activity}

As previously described in Sect. 2.1 of this review, the pro-oxidant action of selenium compounds, including thiol oxidation, RS generation, DNA damage, and mitochondrial dysfunctions, can drive events that culminate in biological downstream effects by affecting kinases, phosphatases, and caspases, proteins involved in the DNA repair and transcription factors that regulate growth, proliferative, and death pathways in cancer cells and different pathogens. Furthermore, organoselenium compounds, particularly ebselen and diselenides, can oxidize critical thiol-containing proteins from viruses, bacteria, and fungi.

Ebselen has been shown to target critical proteins from different viruses due to its reaction with thiols. Toward human immunodeficiency virus type 1 (HIV-1), ebselen was found to be a potent integrase inhibitor, disrupting the interaction of the enzyme with the key growth-factor (lensepithelium-derived growth-factor, LEDGF/p75), through the formation of a selenium-sulfur bond with a cysteinyl residue from the factor LEDGF/p75 (Zhang et al. 2020a). Similarly, the ebselen action as a potent HIV-1 capsid assembly disruptor was associated with its covalent binding to cysteine 198 and 218 residues in the HIV-1 capsid protein (TheninHoussier et al. 2016).

Regarding the hepatitis $\mathrm{C}$ virus, ebselen was recognized as a potent inhibitor of the NS3, a non-structural protein with helicase function. At concentrations higher than $10 \mu \mathrm{M}$, 
ebselen caused an irreversible inhibition and formation of covalent adducts with all cysteines present in the viral helicase (Mukherjee et al. 2014).

Currently, extensive computational-experimental screenings on SARS-CoV-2 have identified several promising drugs that could serve as effective inhibitors of the virus proteins, mainly toward the protease Mpro protease (NSP5), a non-structural sulfhydryl protein involved in the processing of Orf polyproteins 1a and 1ab. The products of hydrolysis of polyproteins 1a and 1ab (NSP4 to NSP 16) are involved in the replication of SARS-CoV-2 as well as SARS-CoV and MERS-CoV (Pillaiyar et al. 2016).

Among various compounds investigated, ebselen appeared as one of the most potent inhibitors of the enzyme both in vitro and SARS-CoV-2 replication in Vero cells. $\mathrm{The}_{\mathrm{IC}}$ for MPro protease was near $0.7 \mu \mathrm{M}$ and for virus replication about $10 \mu \mathrm{M}$ (Jin et al. 2020; Sies and Parnham 2020).

Ebselen covalently attaches to the catalytic cysteine residue from Mpro site active (Cys 145), forming selenosulfide that leads to the enzyme inactivation. Atomistic molecular simulations also provided evidence that ebselen exhibits high-affinity binding for sites localized between the II and III domains of the protein, an important allosteric effect that regulates the enzyme catalytic site (Menendez et al. 2020).

Ebselen and its derivatives have been demonstrated as inhibitors of both Mpro and the papain-like protease (PLPro) from SARS-CoV-2 (Ma et al. 2020); however, they had higher inhibitory effects against Mpro than PLpro (Zmudzinski et al. 2020).

The anti-viral properties of diphenyl diselenide and its derivatives have been only rarely explored. Therefore, diphenyl diselenide has been reported to have virucidal and antiviral actions against in vitro herpes simplex virus 2 (HSV-2), reducing the infectivity in $70.8 \%$ and $47 \%$, respectively. Moreover, treatment with diphenyl diselenide was proven to be effective against oxidative stress and inflammation in HSV-2-infected mice (Sartori et al. 2016, 2017).

In this way, a recent high-throughput screening of a series of new anti-viral diphenyl diselenide derivatives against human herpes virus type 1 (HHV-1) and encephalomyocarditis virus (EMCV) in A549-infected cells revealed their effectiveness against the two viruses. The majority of compounds tested, especially bis[2-(hydroxyphenylcarbamoyl)] phenyl diselenide, exhibited high activity against HHV-1 and moderate activity against EMCV. The anti-HHV-1 activity of most effective diselenides ranged between 2 and $40 \mu \mathrm{g} \mathrm{mL}^{-1}$ (Giurg et al. 2017). Recently, diphenyl diselenide was reported to be effective against bovine alphaherpesvirus 2 (BoHV-2), the agent of bovine herpetic mamillitis, both in vitro and in vivo in ewes transdermally infected with BoHV-2 (Amaral et al. 2020).

\section{Antimicrobial activity}

In the last decades, several studies regarding the antimicrobial activity of organoselenium compounds, such as ebselen, diphenyl diselenide, and selenide-based compounds, toward pathogenic fungi and bacteria have appeared in the literature (Di Leo et al. 2019).

Data from different laboratories have indicated that ebselen and various ebsulfur derivatives exhibited high efficacy against several kinds of clinically relevant fungal strains, among them Candida albicans, Candida glabrata, Candida tropicalis, and Candida parapsilosis. From these studies, the minimal inhibitory concentration (MIC) values found to ebselen ranged from $\sim 0.5$ to $2 \mu \mathrm{g} \mathrm{mL}{ }^{-1}$, whereas $\sim 0.02$ to $12 \mu \mathrm{g} \mathrm{mL}^{-1}$ was reported to its derivatives (Di Leo et al. 2019; May et al. 2018; Ngo et al. 2016; Thangamani et al. 2017). Moreover, ebselen and its structural derivatives, such as benzisoselenazol-3(2H)-one, and 2-methyl- and 2-n-propyl-benzisoselenazol derivatives, 2-phenylbenzisothiazol$3(2 \mathrm{H})$-one, and 2-phenyl-7-azabenzisoselenazol-3(2H)-one, exhibited similar inhibitory activity in assays with fluconazole-resistant strain of C. albicans (Orie et al. 2017).

Ebselen appeared as one of the most active compounds in studies that screened repurposing off-patented molecules with anti-fungal activity against Candida auris (De Oliveira et al. 2019; Wall et al. 2018).

The activity of ebselen nanoencapsulated was markedly increased against $C$. parapsilosis, $C$. albicans, and $C$. tropicalis when compared with the free form (Jaromin et al. 2018). In this way, Vartak and collaborators demonstrated the efficacy of a soluble vaginal film containing ebselen developed to treat vulvovaginal candidiasis, which exhibited an MIC value of $20 \mu \mathrm{M}$ against Candida species, a concentration significantly lower when compared to classical anti-fungal as fluconazole (MIC $500 \mu \mathrm{M}$ ) and miconazole (MIC $100 \mu \mathrm{M}$ ) (Vartak et al. 2020b). The same group also showed the superior efficacy of a novel topical nanoemulgel of ebselen against $C$. albicans and $C$. tropicalis when compared to the effect of a clinically used drug terbinafine that was ineffective even at $100 \mu \mathrm{M}$ (Vartak et al. 2020a).

Ebselen has also been suggested by several studies as a promising molecule to treat bacterial infections alone or in combination with other agents. The effectiveness of ebselen and its derivatives has already been demonstrated against diverse pathogens, including Staphylococcus ssp., Streptococcus ssp., and Enterococcus ssp. (Chen and Yang 2019; Thangamani et al. 2015a).

Ebselen exhibited a potent bactericidal activity against Staphylococcus aureus multidrug-resistant clinical isolates and reduced the bacteria load in a mouse model of staphylococcal skin infections, acting also synergistically with traditional antimicrobials (Thangamani et al. 2015b). 
After an evaluation against a broad array of enterococcal isolates in vitro, ebselen was uncovered as a promising agent for decolonization of vancomycin-resistant enterococci from the gastrointestinal tract (AbdelKhalek et al. 2018).

By targeting cysteine residues in the active site from critical enzymes of Mycobacterium tuberculosis (antigen 85 complex; transpeptidase Ldt Mt2), ebselen and some derivatives were considered promising for treating tuberculosis (de Munnik et al. 2019; Favrot et al. 2013; Goins et al. 2017).

Similarly, from a high-throughput screening assay, ebselen was identified as a potent inhibitor of anthrax receptor (tumor marker endothelial 8, TEM8), via modification of a cysteine residue in the extracellular domain from the anthrax receptor (Cryan et al. 2013). In addition to the receptor modulation, Gustaffon and collaborators demonstrated that ebselen and its derivatives strongly inhibited Bacillus anthracis thioredoxin reductase (Gustafsson et al. 2016).

Screening ebselen and its derivatives for the treatment of ureolytic bacterial infections has revealed these organoselenium compounds as inhibitors of urease activity from Sporosarcina pasteurii and Helicobacter pylori through the interaction with a critical cysteine located at the entrance of the enzyme active site (Macegoniuk et al. 2016).

Very recent studies have demonstrated the synergistic therapeutic efficacy of ebselen and silver against the multidrug-resistant bacteria, including Acinetobacter baumannii, Escherichia coli, and S. aureus (Chen et al. 2019; Dong et al. 2020; Wang et al. 2020).

Increasing evidence indicates the effectiveness of diphenyl diselenide, alone or as adjuvant with classical anti-fungal agents, against diverse strains of fungi. Diphenyl diselenide was effective against 32 Aspergillus isolates (MIC $64 \mu \mathrm{g} \mathrm{mL}^{-1}$ ), which increased the fungicidal action of the drug caspofungin, but was ineffective against an aspergillosis mouse model (Melo et al. 2020).

In vitro, diphenyl diselenide was tested against nineteen Pythium insidiosum isolates and showed an MIC ranging from $\sim 0.5$ to $2.0 \mu \mathrm{g} \mathrm{mL}^{-1}$, the fungistatic activity was reproduced in an animal model of pythiosis (Loreto et al. 2012). Moreover, diphenyl diselenide also increased the efficacy of flucytosine against 30 clinical isolates of Cryptococcus spp. (Rossato et al. 2019).

On clinical $C$. glabrata strains, the diphenyl diselenide MIC ranged from 0.25 to $>64\left(5.16 \mu \mathrm{g} \mathrm{mL}^{-1}\right)$, values similar to that of found for fluconazole. Besides, a synergistic interaction was observed between diphenyl diselenide and the drug amphotericin B (Denardi et al. 2013).

Similar action profile was found against 40 clinical isolates of Sporothrix brasiliensis, in which diphenyl diselenide presented an MIC ranging from 4 to $32 \mu \mathrm{g} \mathrm{mL}^{-1}$ and a synergistic interaction with itraconazole (73\%) (Poester et al. 2019).
Along with diphenyl diselenide, various other diselenide derivatives have been pointed out as effective inhibitors of growth and biofilm formation in fungi and bacteria. Herein we highlighted the compounds camphor diselenide, 2,2'-dithienyl diselenide, bis [ethyl- $N$-(2'-selenobenzoyl) glycinate], bis[2'-seleno- $N$-(1-methyl-2-phenylethyl) benzamide], bis[2-(hydroxyphenylcarbamoyl)]phenyl diselenide, and (Z,Z)-3,30-(4-(diseleno)phenylcarbamoyl)acrylic acid, that in addition to $C$. albicans also showed antibiofilm activity against several bacteria strains, including Enterococcus spp., Staphylococcus spp., Streptococcus ssp., and Pseudomonas ssp. (Bueno Rosseti et al. 2014; Giurg et al. 2017; Pesarico et al. 2013; Rosseti et al. 2015; Sancineto et al. 2016; Shaaban et al. 2015).

There are also some studies comparing the anti-fungal potential of ebselen and diphenyl diselenide, alone or in combination with anti-fungal agents. One of them evaluated the effect of both compounds in combination with amphotericin B, caspofungin, itraconazole, and voriconazole against 25 clinical isolates of Fusarium spp. The MICs found for diphenyl diselenide and ebselen were 4-32 $\mu \mathrm{g} \mathrm{mL}^{-1}$ and 2-8 $\mu \mathrm{g} \mathrm{mL}^{-1}$, respectively. The most effective synergic combinations were found to ebselen + amphotericin B $(88 \%)$, ebselen + voriconazole $(80 \%)$, diphenyl diselenide + amphotericin B (72\%), and diphenyl diselenide + voriconazole (64\%) (Venturini et al. 2016).

Likewise, a comparative study toward $C$. parapsilosis showed that ebselen presented higher anti-fungal activity than diphenyl diselenide against both echinocandin-susceptible and -resistant strains (Chassot et al. 2016). The efficacy of both compounds was also addressed against Trichosporonasahii strains, in which ebselen exhibited an MIC ranging from $\sim 0.25$ to $8 \mu \mathrm{g} \mathrm{mL}^{-1}$ and diphenyl diselenide from $\sim 8$ to $64 \mu \mathrm{g} \mathrm{mL}^{-1}$ (Felli Kubiça et al. 2019).

It is worth mentioning that the mechanisms of antimicrobial action proposed for these organoselenium compounds generally involve similar effects for both fungi and bacteria. Overall, the studies about the antimicrobial activity of different organoselenium compounds have revealed their effectiveness in increasing cell membrane permeability, inhibiting sulfhydryl enzymes, and inducing redox dyshomeostasis in the cells mainly through GSH depletion and RS overproduction, events that can culminate in death (Di Leo et al. 2019).

\section{Chemopreventive activity}

A Janus-faced character of the element selenium, initially classified as carcinogenic (Nelson et al. 1943) and, subsequently, as anticarcinogenic (Shamberger and Frost 1969), has a long history. In that, some of the recent chapters based on clinical trials, cohort, and epidemiological studies have shown an inverse association between selenium 
intake and risk of cancers in humans. Because an extensive discussion of the literature on this field is outside of the scope of this review, the readers are directed to some comprehensive reviews on this topic (Hatfield et al. 2014; Jablonska and Vinceti 2015; Rayman 2020; Stolwijk et al. 2020).

Since the pioneering studies on the anti-cancer activity of organoselenium compounds (El-Bayoumy 1985; Fiala et al. 1991; Nayini et al. 1989; Reddy et al. 1985, 1987; Tanaka et al. 1985), basic research on this topic has moved ahead at a rapid pace, bringing perspectives in cancer prevention and promotion, cancer drug resistance, and molecular mechanisms behind these effects (Chen et al. 2020; Gopalakrishna et al. 2018; Spengler et al. 2019). However, no attempt is made here to thoroughly discuss the beginning studies on chemopreventive effects of organoselenium, as these have been reviewed elsewhere (Nogueira and Rocha 2011; Nogueira et al. 2004).

With regard to ebselen, this organoselenium compound has been proposed to induce RS formation, calcium dyshomeostasis, Bax activation, and mitochondria-mediated apoptosis in different tumor cells, including multiple myeloma, pancreatic tumor, prostate, and leukocytes cancer lines (Gandin et al. 2018; Hanavan et al. 2015; Kaczor-Keller et al. 2020; Santofimia-Castaño et al. 2018). Besides, a very recent study reported that ebselen is an inhibitor of the 6-phosphogluconate dehydrogenase in leukemia cells, an enzyme essential for cell proliferation and tumor growth (Feng et al. 2020). Ebselen was proven to be a potent inhibitor of cell growth for the triple-negative model of breast cancer (Jupp and Giles 2012).

Ebselen and its derivatives have been also indicated as inhibitors of histone deacetylases in tumor cells (Wang et al. 2017b) and effective in reducing cancer cell migration and invasion by targeting multiple kinases with established roles in cancer progression (Bijian et al. 2012).

Thioredoxin reductase 1 (TrxR1)-based drugs have been proposed as promising anti-cancer therapies, because the overexpression of this selenoprotein has been detected in many human tumors. Herein, we highlight the compound ethaselen, an ebselen derivative, which has been pointed out in both in vitro and in vivo studies as a potent anti-proliferative drug, by inhibiting TrxR1 in various types of tumors (Wang et al. 2011a, 2012; Wu et al. 2020). This promising action motivated the use of ethaselen in phase I clinical trial in China, which includes patients diagnosed with advanced non-small-cell lung cancer. The phase $1 \mathrm{a} / 1 \mathrm{~b}$ finished in 2008, and currently, the compound will pass to phase 1c, where the patients will receive oral ethaselen tablets as treatment (600 mg/bid day) (Clinical Trials.gov Identifier: NCT02166242). Moreover, a number of methodological strategies have been carried out for synthesizing ethaselen derivatives with antitumoral activity (Ye et al. 2016).
In addition to $\operatorname{TrR} 1$, selenoprotein 15 (sep15) and GPx2 have been highlighted as important cellular redox regulators potentially involved in preventing and promoting cancer; however, the role of selenoproteins in cancer will be not addressed herein, but interested readers may refer to a comprehensive review published by Hatfield and collaborators (Hatfield et al. 2014).

In the search for novel organoselenium compounds with chemopreventive activity, a class of zidovudine (AZT)-based selenides, named chalcogenozidovudines, was screened as antitumoral candidates against human bladder carcinoma. This study uncovered 5'-(phenylseleno)zidovudine and its $p$-methyl and $p$-chloro derivatives as antitumor agents with potent apoptosis induction effects (de Souza et al. 2015). After a toxicological screening, the $p$-methyl derivative emerged as the most promising candidate for further antitumor studies by exhibiting lower toxicity than AZT on health immune cells and acute in vivo treatment (Ecker et al. 2017).

Increasing evidence indicates the chemopreventive activity of diselenides, especially diphenyl diselenide; as a result, one of the first related studies showed the effectiveness of diphenyl diselenide in inducing death in the human neuroblastoma cell line SH-SY5Y via ERK1/2-mediated apoptosis (Posser et al. 2011). The potential cytotoxic effects of diphenyl diselenide and diphenyl diselenide-loaded nanocapsules have also been reported against C6 glioma cells, in which both forms attenuated the tumor development. Similar results were observed against SK-Mel-103, a resistant melanoma cell line. In these studies, diphenyl diselenide at both forms caused a loss of viability, increased propidium iodide uptake, and nitrite levels in the malignant cells (Ferreira et al. 2019a, 2020).

A detailed discussion of the anti-proliferative activity of diselenide derivatives in malignant cells has been reviewed by others (Álvarez-Pérez et al. 2018; Gandin et al. 2018), and these reviews bring a list of compounds indicated as promising antitumor agents. Briefly, along with diphenyl diselenide on murine hepatoma cells (Hepa 1c1c7), the antiproliferative potential of various diaryl, dialkyl, dipyridazinyl, dipyridinyl, and phenylcarbamate diselenides against other carcinoma cells have been addressed (Álvarez-Pérez et al. 2018; Gandin et al. 2018).

In terms of molecular mechanisms, cell-cycle arresting, caspase-independent and dependent apoptosis, p53 activation, and autophagy via c-Jun N-terminal kinase (JNK) activation are among the cytotoxic effects reported for diorganyl diselenides, including symmetric aromatic diarylseleno and acylselenourea derivatives as well as $m$-trifluoromethyldiphenyl diselenide, $p$-methoxyl-diphenyl diselenide, and diphenyl methylselenocyanate (Chakraborty et al. 2016; Díaz et al. 2018; Garnica et al. 2018).

Furthermore, one way by which the majority of agents, such as ionizing radiation, chemotherapeutic agents, and 
some targeted therapies, kill cancer cells consists of directly or indirectly generating reactive oxygen species (ROS) that block the key steps in the cell cycle (Watson 2013). Therefore, the use of antioxidant dietary supplements, and, consequently, the search for organoselenium compounds that could counteract the ROS generation and prevent tissue damage, allowing an increase in the maximum therapeutic dose of the anti-cancer drug, have been a matter of research interest (Panchuk et al. 2014, 2016).

In experimental models of cisplatin chemotherapy, ebselen has been proven to reduce ovarian damage and ototoxicity through modulation of oxidative injury and apoptosis (Orzáez et al. 2014; Soyman et al. 2018), whereas a naphthalimide-based organoselenium compound enhanced cisplatin antitumor efficacy and reduced its toxicity (Ghosh et al. 2015).

In an MCF-7-cultured cell model, diphenyl diselenide antigenotoxic activity has been associated with the prevention of cancer risk induced by tamoxifen hormone therapy (Melo et al. 2013). Besides, a synergistic antitumor action was identified when ebselen was associated with radiotherapy, which was attributed to the induction of apoptosis and inhibition of breast cancer cell progression (Thabet and Moustafa 2017).

Over the past decades, basic research in the anti-cancer potential of naturally occurring organoselenium compounds has made progress (Chen and Wong 2009; Ip and Ganther 1992; Jiang et al. 1999; Lu et al. 1995; Reddy et al. 2000; Sinha et al. 1999; Sinha and Medina 1997; Unni et al. 2005, 2001), and this knowledge has been translated with some success to clinical trials (Clark et al. 1996; Duffield-Lillico et al. 2003; Lippman et al. 2009; Mix et al. 2015a, b).

Aiming to use higher doses of chemotherapy and overcome drug resistance, the oral bioavailable methylselenocysteine has been investigated in combination with chemotherapeutic agents and proven to be effective against organ-specific toxicities induced by cyclophosphamide, cisplatin, and oxaliplatin, and to enhance antitumor activity in animal models of cancer (Cao et al. 2004, 2014). Very recently, a phase I randomized double-blinded study compared methylselenocysteine and selenomethionine pharmacodynamic effects in cancer patients to determine a safe and effective dose to be used in combination with anti-cancer therapy. However, the dose of $400 \mu \mathrm{g}$ was considered too low to achieve the levels of selenium in plasma ( $\geq 5 \mu \mathrm{M})$, which are expected to cause pharmacodynamic effects (Evans et al. 2020).

Previously published studies revealed that selenomethionine, the organic form of selenium used SELECT trial (Lippman et al. 2009), is ineffective against prostate cancer models (Li et al. 2008; Wang et al. 2009), whereas methylselenocysteine, classified in the second generation of organoselenium compounds, reduces tumor growth and castration-resistant progression of prostate tumor (Christensen et al. 2013; Liu et al. 2015b; Zhan et al. 2013). Methylselenocysteine is considered the most effective among the other natural occurring selenium-containing molecules, because it is efficiently converted to the active intermediate methylselenol, requiring one-step activation by $\beta$-lyase, and does not get as easily serum protein interaction (Bhattacharya 2011; Ip 1998; Ip et al. 2000). Moreover, the anti-cancer effectiveness of methylselenocysteine and selenomethionine has been associated with the transamination reactions that generate $\alpha$-keto acid selenium metabolites, which are potent inhibitors of histone deacetylases (Kassam et al. 2011; Lee et al. 2009; Pinto et al. 2014).

In addition to methylselenocysteine, methylseleninic acid has been reported as a direct precursor of methylselenol, the key metabolite responsible for selenium's anti-cancer activity (El-Bayoumy and Sinha 2004), and effective against prostate cancer (Zhao et al. 2004). By inducing lipid peroxidation, methylseleninic acid sensitizes head-neck squamous cell carcinoma to radiation (Lafin et al. 2019).

Some of the molecular mechanisms underlying chemopreventive activity of naturally occurring organoselenium compounds described so far are the modulation of antioxidant defenses (selenoenzymes) and redox status of cells, programmed cell death, DNA repair, carcinogen detoxification, immune system, neo-angiogenesis, regulation of cell proliferation, and tumor cell invasion (Jung et al. 2013; Korbut et al. 2018; Pons et al. 2020; Wang et al. 2018a; Weekley et al. 2012; Whanger 2004; Zeng and Combs 2008; Zeng et al. 2011).

\section{Antidepressant- and anxiolytic-like activities}

Ebselen, a safe and well-tolerated organoselenium (Lynch and Kil 2009; Parnham and Graf 1987), has attracted tremendous interest over the past decades. Although it never reached the market, the possibility to apply for novel applications or repurposing ebselen is at the core of basic academic research and clinical trials (Kil et al. 2017; Ogawa et al. 1999; Parnham and Sies 2013; Saito et al. 1998; Sharpley et al. 2020; Yamaguchi et al. 1998). In 2013, drugrepurposing studies indicated ebselen as a mood stabilizer; this is because it is an inhibitor of inositol monophosphatase (IMPase), induces lithium-like effects on mouse behavior, crosses the blood-brain barrier, down-regulates serotonin (5-HT) 2A receptor in the head-twitch and c-Fos models, inhibits glutamine synthase, and decreases myo-inositol concentration in the human brain (Masaki et al. 2016a, b; Singh et al. 2013, 2016).

More recently, ebselen has been suggested as an antidepressant (Antoniadou et al. 2018) and impulsivity lowering agent (Barkus et al. 2018) based on its neuropharmacological lithium-like effects in different animal models 
of 5-HT2A function. Therefore, Fink and collaborators reported that elevated 5-HT2A receptor expression is associated with impulsive behavior in rodents (Fink et al. 2015).

The success of ebselen in experimental models of mood disorders (Posser et al. 2009) has encouraged the study of other organoselenium compounds that share with this compound some properties relevant to the antidepressant- and anxiolytic-like activities such as neuroprotective, antiinflammatory, and the modulation of neurotransmitter systems (Nogueira et al. 2003; Nogueira and Rocha 2011).

The antidepressant-like property of diphenyl diselenide, a structurally simple compound, was first demonstrated by our research group in 2007 (Savegnago et al. 2007b) and, since that, substituted diorganoyl diselenides have been reported as effective agents in different animal models of depression (Acker et al. 2009; Brüning et al. 2015b; Da Rocha et al. 2012a; Dias et al. 2014; Quines et al. 2016b; Savegnago et al. 2008b).

Table 2 illustrates some protocols in which diaryl diselenides were effective at relatively low doses in rodents (Bortolatto et al. 2012; Brüning et al. 2011; Heck et al. 2019; Oliveira et al. 2017; Zborowski et al. 2020), excepting $m$-trifluoromethyl-diphenyl diselenide that was reported to be effective at minimal $50 \mathrm{mg} \mathrm{kg}^{-1}$ dose in an acute protocol (Brüning et al. 2011). However, the $m$-trifluoromethyldiphenyl diselenide effective antidepressant-like dose was reduced by tenfold when it was administered once daily for 8 days in mice (Rosa et al. 2017) and substantially decreased in a TNF- $\alpha$ model of depression (Brüning et al. 2015b). Moreover, this substituted diaryl diselenide has been reported to promote resilience to social avoidance induced by social defeat stress (Rosa et al. 2018c) and abolish the depressant-like phenotype induced by repeated forced swim stress (Rosa et al. 2018b).

Evidence has been found to suggest that the simple substitution of hydrogen by the $m$-trifluoromethyl group on diphenyl diselenide generates an organoselenium compound that, different from the parent compound, modulates the opioid system in different animal models of mood disorders (Brüning et al. 2011; Martins et al. 2020; Rosa et al. 2017, 2018b). However, neither $m$-trifluoromethyl- nor $p$-methoxyl- diaryl diselenide was associated with anxiety phenotype after discontinuation (Oliveira et al. 2017) and withdrawal syndrome in mice (Rosa et al. 2017), which are experienced by users of antidepressant drugs.

We highlight herein the ambiguity of results obtained with the simplest diaryl diselenide, diphenyl diselenide, in different experimental models of anxiety that undoubtedly demonstrate its well-documented Janus-faced characteristic (Nogueira and Rocha 2010). Results from different groups of research indicate that diphenyl diselenide produced an anxiolytic-like effect in naive (Ghisleni et al. 2008a) and monosodium glutamate-exposed rats (Rosa et al. 2016), naive mice (Savegnago et al. 2008b), naive (Ibrahim et al. 2014b), and hyperglycemic zebrafish (dos Santos et al. 2018), and chick subjected to social separation-stress (Prigol et al. 2011). On the other hand, administration of diphenyl diselenide after repetitive mild traumatic brain injury exacerbated anxiety-like response in rats, increasing the telomeres and the levels of TNF $\alpha$ (Yamakawa et al. 2020). Moreover, in Carioca high- and low-conditioned freezing rats, an animal model of generalized anxiety, diphenyl diselenide induced both anxiogenic- and anxiolytic-like effects (Hassan et al. 2015).

Table 2 Potential antidepressant-like activity of diaryl diselenides

\begin{tabular}{|c|c|c|c|c|c|c|}
\hline & Experimental model & $\begin{array}{l}\text { Minimal } \\
\text { effective } \\
\text { dose }\end{array}$ & Treatment & Predictive tests & Species & References \\
\hline \multirow[t]{2}{*}{ Diphenyl diselenide } & Methimazole & $5^{\mathrm{a}}$ & 90 days & FST & Rats & Dias et al. (2014) \\
\hline & Monosodium glutamate & 10 & $30 \mathrm{~min}$ & FST & Rats & Quines et al. (2016b) \\
\hline \multirow{3}{*}{$\begin{array}{l}p \text {-Chloro- } \\
\text { diphenyl diselenide }\end{array}$} & - & 10 & 7 days & FST & Old rats ${ }^{\mathrm{b}}$ & Bortolatto et al. (2012) \\
\hline & Streptozotocin & 5 & 7 days & FST, TST & Mice & Zborowski et al. (2020) \\
\hline & Dexamethasone & 5 & 7 days & FST, TST & Mice & Heck et al. (2019) \\
\hline \multirow{3}{*}{$\begin{array}{l}m \text {-Trifluoromethyl } \\
\text { diphenyl diselenide }\end{array}$} & - & 50 & $30 \mathrm{~min}$ & FST & Mice & Brüning et al. (2011) \\
\hline & - & 5 & 8 days & FST, mTST & Mice & Rosa et al. (2017) \\
\hline & TNF- $\alpha$ & 0.1 & $30 \mathrm{~min}$ & FST, TST & Mice & Brüning et al. (2015b) \\
\hline \multirow[t]{2}{*}{ p-Methoxyl-diphenyl diselenide } & - & 5 & $30 \mathrm{~min}$ & mFST & Mice & Sartori et al. (2017) \\
\hline & - & 0.1 & 7 days & $\begin{array}{l}\text { TST } \\
\text { mFST }\end{array}$ & Mice & Sartori et al. (2017) \\
\hline
\end{tabular}

Diselenides $\left(\mathrm{mg} \mathrm{kg}^{-1}\right)$ were intragastrically administered, excepting ${ }^{\mathrm{a}}$ that was added in the diet (ppm/diet)

Depression predictive tests: FST forced swimming test, TST tail suspension test, $m F S T$ modified forced swimming test, $m$ TST modified tail suspension test, $T N F$ tumoral necrosis factor, ${ }^{\mathrm{b}} 23$ months, rats (Wistar), mice (Swiss) 
The potential of selenides in tests predictive of depression and anxiety has received some attention over the past decade (Besckow et al. 2020; Donato et al. 2013; Gerzson et al. 2012; Jesse et al. 2011; Oliveira et al. 2012; Reis et al. 2017a; Sampaio et al. 2020; Victoria et al. 2014). According to Table 3, (octylseleno)-xylofuranoside challenged in the mouse TST produced an antidepressant-like activity at a very low dose. The minimal effective dose of (octylseleno)xyloruranoside was, at least, 100-fold lower (Brod et al. 2016) than those of the other selenides shown in Table 3. Of note, ebselen (3-30 $\mathrm{m} \mathrm{kg}^{-1}$ ) did not produce an antidepressant-like activity in the TST (Posser et al. 2009).

A series of selenophene derivatives were evaluated as potential antidepressant molecules (Gai et al. 2012); among them, 3-( $p$-fluorophenylselenyl)-2,5-diphenylselenophene was reported to be effective in naive mice as well as in corticosterone and partial sciatic nerve ligation (PSNL) models of depression (Gai et al. 2014a, b, c; Gay et al. 2010). When it was subchronically administered, the anxiolytic-like property of 3-( $p$-fluorophenylselenyl)-2,5-diphenylselenophene was demonstrated in corticosterone (Gai et al. 2014b), shamoperated, and PSNL mice (Gai et al. 2014a) (Table 3).

Further investigations of organoselenium compounds, that similar to serotonin contain the indole nucleus, uncovered 1-methyl-3-(phenylselanyl)- $1 \mathrm{H}$-indole as an antidepressant- and anxiolytic-like molecule in the icv streptozotocin model (Bampi et al. 2019, 2020b) (Table 3).

Besides, 1-methyl-3-(phenylselanyl)-1 $H$-indole was reported to be effective against depressive-like behavior and hyperglycemia in a model of type 1 diabetes induced by streptozotocin in mice (Bampi et al. 2020a). The introduction of the $p$-chloro substituent at the-phenylselanyl group affords 3-[(p-chlorophenyl)selanyl]-1-methyl- $1 H$-indole, an antidepressant-like compound effective against lipopolysaccharide-induced depression- and anxiogenic-like phenotype, without promoting locomotor impairment in mice (Casaril et al. 2017, 2019b). It was demonstrated that this substituted selenium indole derivative was also effective in the acute restrain stress-induced depressive-like behavior in mice (Casaril et al. 2019a).

In the search for new potential antidepressant-like compounds, the same group synthesized and demonstrated the antidepressant activity of another $N$-heterocycle derivative, 3-((p-methoxyphenyl)selanyl)-2-phenylimidazo[1,2a]pyridine (Domingues et al. 2018, 2019). Very recently, an isoquinoline derivative has been reported as an antidepressant-like compound that selectively and reversibly inhibited cerebral monoamino oxidase (MAO) B activity in C57Bl/6 mice (Sampaio et al. 2016, 2020).

It may be mentioned that the drug interaction with multiple targets, instead of a single one, has raised considerable interest in the treatment of complex diseases as mood disorders (Koeberle and Werz 2014). On the other hand, the lack of target specificity, possibly because organoselenium compounds react with a multitude of protein thiols, could result in drugs potentially good for everything but effective for nothing. Therefore, the proposed mechanisms to explain the antidepressant- and anxiolytic-like properties of the above-reported organoselenium compounds indicate multiple molecular sites of action; most of them demonstrated using empirical and non-robust methodologies.

Table 3 Potential antidepressant-like activity of selenides

\begin{tabular}{|c|c|c|c|c|c|}
\hline & \multirow[t]{2}{*}{ Experimental model } & \multirow{2}{*}{$\begin{array}{l}\text { Minimal } \\
\text { effective } \\
\text { dose }\end{array}$} & \multicolumn{2}{|l|}{ Predictive tests } & \multirow[t]{2}{*}{ References } \\
\hline & & & Depression & Anxiety & \\
\hline $\begin{array}{l}\text { (Octylseleno) } \\
\text { xilofuranoside }\end{array}$ & - & 0.001 & TST & - & Brod et al. (2016) \\
\hline \multirow{3}{*}{$\begin{array}{l}\text { (Fluorophenylselenyl)-2,5-diphenyl } \\
\text { selenophene }\end{array}$} & - & 50 & FST & - & Gay et al. (2010) \\
\hline & Corticosterone & $0.1^{\mathrm{a}}$ & FST, TST & $\mathrm{LD}, \mathrm{NSF}$ & Gai et al. (2014b) \\
\hline & Partial sciatic nerve ligation & 1 & FST, TST & LD & Gai et al. (2014a) \\
\hline \multirow{2}{*}{$\begin{array}{l}\text { 1-Methyl-3-(phenylselanyl)-1H- } \\
\text { indole }\end{array}$} & \multirow{2}{*}{$\begin{array}{l}\text { Streptozotocin (intracerebro ven- } \\
\text { tricular) }\end{array}$} & 10 & FST, Splash & - & Bampi et al. (2020b) \\
\hline & & $10^{\mathrm{a}}$ & $\begin{array}{l}\text { Social interact, TST } \\
\text { Splash }\end{array}$ & EPM & Bampi et al. (2019) \\
\hline \multirow[t]{2}{*}{$\begin{array}{l}\text { (Chlorophenyl)selanyl]-1-methyl- } \\
1 H \text {-indole }\end{array}$} & Lipopolysaccharide & 1 & $\begin{array}{l}\text { TST } \\
\text { Splash }\end{array}$ & $\begin{array}{l}\text { EPM } \\
\text { MB }\end{array}$ & Casaril et al. (2019b) \\
\hline & Acute restrain stress & 1 & $\begin{array}{l}\text { TST } \\
\text { Splash }\end{array}$ & - & Casaril et al. (2019a) \\
\hline
\end{tabular}

Selenides $\left(\mathrm{mg} \mathrm{kg}^{-1}\right)$ were intragastrically administered to Swiss mice 30 min before tests, excepting athat means repeated treatment ( 7 days), FST forced swimming test, TST tail suspension test, $L D$ light dark test, $N S F$ novelty suppressed-feeding, $E P M$ elevated plus maze, $M B$ marble burying 


\section{Anti-inflammatory and anti-nociceptive activities}

Over the last decades, the anti-inflammatory properties of ebselen have been well recognized in cellular systems and studies with laboratory animals (Aruoma 1997; Baek et al. 2016; Brüne et al. 1991; Cotgreave et al. 1988, 1989; Dimmeler et al. 1991; Gao and Issekutz 1993; ICHIKAWA et al. 1987; Kuhl et al. 1986; Leurs et al. 1989; Safayhi et al. 1985; Schewe et al. 1994; Zembowicz et al. 1993; Zhang et al. 2002), and the pioneer studies were previously summarized elsewhere (Nogueira and Rocha 2011; Nogueira et al. 2004; Schewe 1995) and need not to be repeated here.

Although a vast body of literature supports the potential use of ebselen as a drug that in addition to its antioxidant property acts in multiple cellular mechanisms involved in the inflammatory process (Parnham et al. 1991; Schewe 1995; Smith et al. 2012; Xu et al. 2018), its medical application in inflammatory human diseases is until expected. One plausible explanation for the lack of ebselen effectiveness in chronic inflammatory diseases is that ROS cause endothelial dysfunction and tissue damage (Halliwell 2012), but can also help to resolve it (Halliwell 2006; Sareila et al. 2011), which illustrates the paradoxical action of antioxidants in certain diseases (Halliwell 2013).

As described earlier, simple diaryl diselenides showed potent anti-inflammatory activity in vitro and in vivo acute inflammatory models (Galet et al. 1994; Nogueira et al. 2003; Shen et al. 2004; Shin et al. 2009). From these studies, structure-activity relationship can be distinguished and indicated $m$-hydroxyphenyl diselenide as a potent anti-inflammatory agent in lipopolysaccharide-activated macrophage cells (Shin et al. 2009).

Shortly after, inspired by the Shin study, the anti-inflammatory properties of diphenyl diselenide were identified in classically activated macrophages. In this study, diphenyl diselenide reduced the expression of NO synthase and, consequently, the NO production, diminished the levels of ROS and arginase activity, and down-regulated classical and alternative activation phenotype of macrophages (Rupil et al. 2012). By modulating pro-inflammatory markers and oxidative stress, diphenyl diselenide has been reported to be effective in a mouse model of carrageenan-induced pleurisy (Luchese et al. 2012b), ischemia and reperfusion-induced cerebral injury (Brüning et al. 2012a), and experimental toxoplasmosis (Barbosa et al. 2014; Doleski et al. 2017a, b).

In an experimental model of ulcerative colitis, repeated treatment with diphenyl diselenide $\left(50 \mathrm{mg} \mathrm{kg}^{-1}\right.$, i.g.) was found to be more effective than ebselen $\left(50 \mathrm{mg} \mathrm{kg}^{-1}\right)$ to reverse colon damage, neutrophil infiltrate, and oxidative stress markers in rats (Petronilho et al. 2016). When supplemented in the diet, diphenyl diselenide reduced the levels of serum pro-inflammatory cytokines (IL-1, TNF- $\alpha$ ) and increased the IL-10 concentration in middle-aged rats and dairy sheep (Biazus et al. 2019; Leite et al. 2015). The modulation of purinergic signaling and nucleotide-binding oligomerization domain-like receptor (NLRP3) inflammasome gene expression was reported as the underlying mechanisms by which diphenyl diselenide induced an anti-inflammatory response in methyl-mercury chloride-induced immunotoxicity in grass carp (Souza et al. 2019). Moreover, by modulating splenic purinergic signaling, diphenyl diselenide reduced inflammatory and hemorrhagic processes induced by fumonisin B1 in silver catfish (Baldissera et al. 2020b).

Recent evidence associates the anti-inflammatory properties of selenomethionine and its hydroxyl analogue with suppression of the NF- $\mathrm{kB}$ pathway and the increase in the expression of selenoproteins encoding genes in trachea and liver of lipopolysaccharide-exposed chicken (Qu et al. 2020; Shi et al. 2020) and spleens of Kunming mice exposed to lipopolysaccharide (Tang et al. 2019).

\section{Antinociceptive activity}

Increasing evidence indicated that redox modulation is important in the sensitization of peripheral nociceptors (Bhave and Gereau 2004; Choi and Lipton 2000; Meotti et al. 2009), supporting the potential of drugs aimed at restoring the redox homeostasis and relieving pain. Therefore, organoselenium compounds have been screened for antinociceptive activity, and some of these findings showed that both diphenyl diselenide and ebselen, systemically or locally administered, were effective in mouse models of nociception (Nogueira et al. 2003; Rosa et al. 2015; Savegnago et al. 2007a, 2008a; Zasso et al. 2005). It is important to note that ebselen, different from diphenyl diselenide (Savegnago et al. 2007c), produced a synergistic pronociceptive effect when administered with glutamate, which was blocked by a reduced glutathione depleting agent (Meotti et al. 2009).

$p$-Methoxyl-, $m$-trifluormethyl-, and $p$-methyl-substituted diaryl diselenides were reported to be as effective as a nonsubstituted diphenyl diselenide in experimental models of nociception (Brüning et al. 2014, 2010; Donato et al. 2015; Jesse et al. 2009; Oliveira et al. 2016; Pinto et al. 2008), suggesting that the characteristics of substituents at the aryl moiety do not alter the antinociceptive property of diselenides (Araujo et al. 2020; Brüning et al. 2015a; Savegnago et al. 2007a).

Over the last decade, considerable efforts have been made to synthesize organoselenium compounds with greater antinociceptive activity and fewer side effects, resulting in a multitude of molecules with relative success, namely imidazole diselenide derivative (Chagas et al. 2013b, 2017a, b), dipyridil diselenide and its $m$-amino-substituted derivative (Reis et al. 2019; Rosa et al. 2018a), salicylic acid-selenium derivative (Chagas et al. 2014), bis-vinylselenide derivatives 
(Jesse et al. 2007, 2008; Savegnago et al. 2006a), selenosteroid derivative (Sari et al. 2014), and quinoline (Pinz et al. 2016), indole (Birmann et al. 2018), and pyrazole (Oliveira et al. 2020) selenide derivatives. However, the organoselenium compounds synthesized so far have low aqueous solubility and pharmacokinetic and toxicokinetic practically unknown (Fischer et al. 1988; Müller et al. 1988; Prigol et al. 2013; Sies 1994), limiting the possibility of their therapeutic applications.

Therefore, the rationale to incorporate $p$-methoxyldiphenyl diselenide in polymeric nanocapsules was based on the enhancing of its pharmacokinetic properties (Sari et al. 2017), and resulted in a more prolonged anti-hypernociceptive action and greater restorative effects than the free compound in a mouse model of neuropathic pain (Sari et al. 2018a, b).

\section{Hepato and gastroprotective activities}

In the earlier 50s, Schwarz and Foltz demonstrated the effectiveness of element selenium against dietary necrotic liver degeneration in rats (Schwarz and Foltz 1957) and, since that, naturally occurring and synthetic organoselenium compounds have been widely investigated as possible hepatoprotective molecules (Brzački et al. 2019; Ibrahim et al. 2010; Jiang et al. 2016; Kono et al. 2001; Ozaki et al. 1997; Reis et al. 2017b; Schwarz and Fredga 1969; Shimohashi et al. 2000; Tiegs et al. 1998; Wang et al. 1992; Wendel and Tiegs 1986; Wilhelm et al. 2010, 2011).

Three decade-long studies on the protective action of ebselen in a diversity of experimental models of hepatotoxicity, such as galactosamine (Wendel and Tiegs 1986), paracetamol (Qiu-Ju et al. 1994; Rocha et al. 2005), $\mathrm{CCl}_{4}$ (Wasser et al. 2001), lipopolysaccharide and Propionibacterium acnes (Koyanagi et al. 2001), alcohol (Oshita et al. 1994; Pivetta et al. 2006), ischemia-reperfusion (Ozaki et al. 1997), manganese (Ismail 2019), and radiocontrast media (Basarslan et al. 2013), have indicated the modulation of NF-kB-dependent pathway and activation of the immune system as the mechanisms accounted for this action (Shimohashi et al. 2000; Tiegs et al. 1998; Wang et al. 1992).

Diphenyl diselenide and its substituted derivatives have been also screened for hepatoprotective activity in 2-nitropropane (Borges et al. 2005, 2006; Ibrahim et al. 2010), metal (Borges et al. 2008; Dalla Corte et al. 2016), acetaminophen (Carvalho et al. 2013, 2017; da Rosa et al. 2012; Wilhelm et al. 2009b, c), bisphenol A (Müller et al. 2018), thioacetamide (Stefanello et al. 2015b), lipopolysaccharide/ galactosamine (Wilhelm et al. 2009a), and organophosphate (Acker et al. 2012; Costa et al. 2013) models of damage in rodents.

Regarding the hepatic injury induced by $\mathrm{CCl}_{4}$, repeated administration of $p$-methylbenzoyl diselenide derivative
(Filho et al. 2013) and selenocystine (Uzma et al. 2011) has proven to be effective, whereas treatment with diphenyl diselenide potentiated $\mathrm{CCl}_{4}$ damage, suggesting that biotransformation of this toxicant by cytochromes $\mathrm{P} 450$ was activated by diphenyl diselenide (Nogueira et al. 2009).

Despite the significance to avoid gastric mucosal irritation in the design of potential anti-inflammatory drugs and the importance of prescribing antisecretory drugs for longterm use of anti-inflammatory therapy (Graham and Chan 2008), the gastroprotective activity of organoselenium compounds has been few explored (Ibrahim et al. 2018) over the last decade. Therefore, the knowledge on this subject is supported by previously published studies, which demonstrated that ebselen and diphenyl diselenide not only reduce $\mathrm{HCl}$ secretion, but also inhibit gastric lesions in a great number of experimental models (Beil et al. 1990; Ineu et al. 2008; Kurebayashi et al. 1989; Leyck and Parnham 1990; Ohta et al. 2002; Savegnago et al. 2006b; Tabuchi and Kurebayashi 1993; Tabuchi et al. 1994, 1995).

\section{Renoprotective activity}

Studies on the renoprotective activity of organoselenium compounds remained to be explored; as a result, most of the literature of the last 2 decades focuses on ebselen and diphenyl diselenide effects on models of renal damage.

With regard to ebselen activity, it protected against ischemic renal injury (Noiri et al. 2001) as well as gentamicin- (Dhanarajan et al. 2006), sodium arsenite- (AlBrakati et al. 2019), sporadic Alzheimer's disease (AD) model- (Klann et al. 2020), and radiocontrast- (Ozgur et al. 2012) induced renal damage in rodents. In a model of renal ischemia/reperfusion damage, ebselen associated with $\mathrm{N}$-acetylcysteine was proven to be effective (Kizilgun et al. 2011).

Using experimental models of diabetes, beneficial effects of ebselen and its $m$-hydroxyl derivative were demonstrated in Zucker diabetic fat rats (Gealekman et al. 2004) and the ApoE/GPx1 double knockout mouse (Tan et al. 2013). Although the authors suggest synthetic mimetics as a useful therapeutic strategy in reducing diabetic complications, late chronic intervention with ebselen reduced oxidative stress, but failed to attenuate functional or structural kidney damage in the Akita mouse model of nephropathy (Tan et al. 2015).

Since its approval by the Food and Drug Administration in 1978, cisplatin has been one of the most used drugs for solid cancer treatments. However, nephrotoxicity is the most well-known and clinically important toxicity of this chemotherapeutic (Ghosh 2019). In an attempt to counteract the main drawback of the cisplatin use, which limits its application, organoselenium compounds have been investigated in models of renal damage induced by this chemotherapy. Ebselen (Lynch et al. 2005; Yoshida et al. 2000), 
p-methoxyl-diphenyl diselenide (Wilhelm et al. 2012), naphtalamide (Ghosh et al. 2015), selenocyanate isoquinoline derivative (Ghosh et al. 2013), and diselenole (Bortolatto et al. 2014) have appeared in the literature as effective organoselenium compounds against cisplatin-associated nephrotoxicity. In a juvenile rat model of cisplatin-induced nephrotoxicity, diphenyl diselenide $\left(12 \mathrm{mg} \mathrm{kg}^{-1}\right.$, i.g.) was reported to be as effective as ebselen $\left(11 \mathrm{mg} \mathrm{kg}^{-1}\right.$, i.g.) in rats (Fulco et al. 2020).

Searching for naturally occurring organoselenium compounds that reduce renal toxicity and enhance the antitumor activity of cisplatin, selenomethionine, and methylselenocysteine was highlighted as promising molecules (Cao et al. 2014; García Sar et al. 2011).

Based on the well-reported effectiveness of diphenyl diselenide against toxicity induced by metals (Brandão et al. 2008; Santos et al. 2005a, b), the potential renoprotective activity of this diselenide was investigated in a mercuric chloride model of renal injury $\left(5 \mathrm{mg} \mathrm{kg}^{-1}\right.$, s.c.). From these studies, the Janus-faced duality of diphenyl diselenide was recognized; an acute dose of $31 \mathrm{mg} \mathrm{kg}^{-1}$ (s.c.) potentiated mercuric chloride-induced nephrotoxicity (Brandão et al. 2011), whereas a 5 -day repeated dose $\left(5 \mathrm{mg} \mathrm{kg}^{-1}\right.$, i.g.) protected against toxic effects of this metal in the kidney of mice (da Luz Fiuza et al. 2015). It is acknowledged that diphenyl diselenide effects on renal injury induced by mercuric chloride depend on the dose of this organoselenium and the protocol of administration of both compounds.

Moreover, diphenyl diselenide $\left(7.14 \mathrm{mg} \mathrm{kg}^{-1}\right.$, i.g.) repeated administration for 7 days protected against glycerol induced nephrotoxicity in rats (Brandao et al. 2009), whereas its binaphthyl derivative $\left(50 \mathrm{mg} \mathrm{kg}^{-1}\right.$, i.g.) was effective at a higher acute dose in mice (Ibrahim et al. 2011).

\section{Cardioprotective activity}

Regarding selenium status, both excess and deficiency are associated with some adverse health conditions (Rayman 2020), and a well-known example relates to cardiovascular disorders and increased mortality in individuals who have selenium-deficient levels (Bleys et al. 2008; Lubos et al. 2010). Further of note, Keshan disease, endemic cardiomyopathy, and Kashin-Beck disease, deforming arthritis, are both associated with selenium deficiency in China (NavarroAlarcon and López-Martınez 2000; Xiong et al. 2010).

Hypercholesterolemia, a very important risk factor to cardiovascular disease, has been related to selenium deficiency, which would lead to the decrease in low-density lipoprotein removal from blood and in apolipoprotein B catabolism, through down-regulation of low-density lipoprotein receptor activity mRNA expression (Dhingra and Bansal 2005, 2006; Lee et al. 2003).
In addition to the significance of selenium to cardiac health maintenance (Gunes et al. 2017; Rocca et al. 2018; Yang et al. 2017), organoselenium compounds have been investigated in models of cardiotoxicity and ischemia/reperfusion injury, in which redox imbalance triggers a number of signaling pathways mediated by reactive oxygen and nitrogen species, and, that antioxidants play a role.

Based on the fact that cardiotoxicity is one of the recognized drawbacks of anti-cancer treatments (Dong and Chen 2018), ebselen was tested and proven to be effective against chemotherapeutic daunorubicin-induced cardiomyopathy in rats (Saad et al. 2006). From experiments with reconstituted human heart, cytosolic fractions emerge the molecular mechanism of ebselen cardioprotective activity, the inhibition of reductive anthracycline alcohol metabolite formation (Mordente et al. 2015).

The suppression of cardiomyocyte apoptosis, anti-inflammatory, and antioxidant activities have been implicated in the cardioprotective action of ebselen against ischemia/reperfusion injury in rats (Cheng et al. 2019; Steinbrenner et al. 2016).

Used as an adjuvant in heart preservation, ebselen was added to a cardioplegic histidine-tryptophan-ketoglutarate (HTK) solution and proven to enhance myocardial protection in a piglet model of cardiopulmonary bypass (Liu et al. 2015a).

Considering that oxidized low-density lipoprotein (LDL) is recognized as a key stage in the development of atherosclerosis, diphenyl diselenide and its substituted diaryl diselenides were investigated in a model of LDL oxidation in vitro, in which the antioxidant and antiatherogenic effects were reported (de Bem et al. 2008; Straliotto et al. 2013a). Shortly after, the same authors demonstrated that diphenyl diselenide reduced oxidized LDL-induced cytotoxicity and down-regulated NF-kB pathway in murine macrophage cells (Straliotto et al. 2013b). In animal models, diphenyl diselenide reduced hypercholesterolemia in cholesterol-fed rabbits (De Bem et al. 2009) and tyloxapol-exposed mice (Da Rocha et al. 2009; Sartori Oliveira et al. 2016). The reduction of atherosclerotic plaque formation and endothelial dysfunction, and antiatherogenic properties of diphenyl diselenide were attributed to the increase of the antioxidant defenses in the cardiovascular system of low-density lipoprotein receptor knockout (LDLr -/-) mice (Hort et al. 2011; Mancini et al. 2014).

Moreover, the cardioprotective activity of diphenyl diselenide was demonstrated in a toxoplasmosis model of cardiac toxicity (Machado et al. 2016).

The naturally occurring organoselenium compound selenomethionine has recently been exploited in HOClinduced dysfunction in myoblastic cells. In this study, cardiac myoblast protection was attributed to the antioxidant activity of selenomethionine. However, selenomethionine 
supplemented $\left(2 \mathrm{mg} \mathrm{kg}^{-1}\right)$ in the diet had limited efficacy in a rat cardiac ischemia/reperfusion injury (Reyes et al. 2019). In an apolipoprotein E-deficient (ApoE -/-) mouse model of atherosclerosis, selenomethionine $\left(2 \mathrm{mg} \mathrm{kg}^{-1}\right)$ added within a high-fat diet-fed decreased the formation of atherosclerotic plaque and M1 inflammatory-type macrophages. The translational applicability of this study was highlighted by the decrease in the extent of extracellular trap release from phorbol myristate acetate (PMA)-stimulated mouse bone marrow-derived cells, which was replicated on cultured neutrophils isolated from acute coronary syndrome patients (Zhang et al. 2020e). Although at a lower concentration in the diet $\left(0.5 \mathrm{mg} \mathrm{kg}^{-1}\right)$, selenomethionine was effective against myocardial oxidative stress and inflammation induced by LPS through the miR-128-3p-p38MAPK-NF-kB pathway in chicken (Liu et al. 2020a).

Cardiovascular injury has been associated with radiation in cancer therapy (Eldabaje et al. 2015), in which the redox modulation plays an important role. Therefore, selenomethionine $\left(4 \mathrm{mg} \mathrm{kg}^{-1}\right)$ was able to protect the rat heart tissue against radiation-induced injury by down-regulating the expression of dual oxidase (Duox1 and Duox2); two important pro-oxidant enzymes (Kolivand et al. 2019).

\section{Insulin-mimetic activity}

The beneficial or harmful effects of selenium on type 2 diabetes are a matter of controversy, resembling the U-shaped behavior of this nutritionally essential trace element in animals and humans. A detailed discussion of the literature on this field is outside of the scope of this review, and has been reviewed by others (Duntas and Benvenga 2015; Kohler et al. 2018; Rayman 2020; Rayman and Stranges 2013; Schomburg 2020).

Despite some controversy, high dietary selenium intake has been associated with protection against obesity and type 2 diabetes (Wang et al. 2016a, 2017a), but the mechanisms to explain this action are unknown or unclear. The demonstration that activation of redox-sensitive thermogenic cascade and the uncoupling protein 1 (UCP1) initiates thermogenesis in brown adipose tissue, a tissue metabolically reprogrammed that requires bioenergetic substrates to increase mitochondrial respiration and produce heat via the UCP1, further support the evidence that selenium could be beneficial in type 2 diabetes and obesity (Lettieri-Barbato 2019).

Very recently, an elegant study, which applied a mass spectrometric tool to identify selenium insertions in proteins, demonstrated the existence of facultative protein selenation, which correlates with impacts on thermogenic adipocyte function. In this study, selenium as selenocysteine $(\mathrm{Sec})$ was selectively incorporated into regulatory sites on key metabolic proteins as an alternative to cysteine at position 253 in the UCP1. It is important to highlight that UCP1-Sec253 is highly sensitive to redox modulation and that increasing the pool of this type of facultative selenoprotein coincides with brown adipose tissue-dependent energy expenditure (Jedrychowski et al. 2020).

In addition to the selenium role as an integral component of several enzymes, such as formate dehydrogenase, glutathione peroxidase, selenoprotein $\mathrm{P}$ and $\mathrm{W}$, and the deiodinases (Beckett and Arthur 2005), the possibility to mimic the insulin action has been reported to help in explaining the antidiabetogenic properties of inorganic forms of this element. For a more in-depth discussion of issues that support selenate as an effective insulinmimetic, readers are directed to a comprehensive review (Stapleton 2000).

Regarding organoselenium compounds, the effectiveness of ebselen and diphenyl diselenide has been reported in a number of experimental models of diabetes, and its complications (Barbosa et al. 2008, 2006; Bubolz et al. 2007; Chander et al. 2004; De-Mello et al. 1996; Gealekman et al. 2004; Kade et al. 2009; Soares et al. 2014; Zhou et al. 2016).

Over the last 10 years, most of the research has focused on the underlying mechanisms of ebselen antidiabetogenic properties. Experimental models in which ebselen, besides reducing hyperglycemia, stimulates insulin secretion (Wang et al. 2014) and hepatic glycogen synthesis (Costa et al. 2012), modulates expression of glucose transporter (GLUT2) and glucokinase, suppresses gluconeogenesis by decreasing phosphoenolpyruvate carboxykinase (PEPCK) expression (Park et al. 2014), and prevents islet apoptosis as well as preserves mass and function of $\beta$-cells, by reducing oxidative stress markers and enhancing intranuclear location of critical insulin transcription factors (Mahadevan et al. 2013; Sasaki et al. 2013), are listed in Table 4.

However, when evaluated against oxidative stress-induced endothelium-dependent vasodilation in diabetic patients, ebselen was proven to be ineffective, at least at the dose tested in this trial (150 mg per oral, twice daily) (Beckman et al. 2016).

Diphenyl diselenide insulin-like properties have been associated with the increase in low-density lipoprotein receptor (LDLr) expression and the translocation of glucose transporter (GLUT4) through the adenosine monophosphate kinase (AMPK) activation (da Rocha et al. 2013a, b), the decrease of cholesterol levels and reduction of visceral fat (da Rocha et al. 2011; Ribeiro et al. 2013), and the modulation of gluconeogenesis enzymes, in addition to regulating hyperglycemia (Acker and Nogueira 2014). Recently, a study from our research group demonstrated that the anti-hyperglycemic effect of diphenyl diselenide is associated with an up-regulation of insulin receptor and glucose transporter driven by the product of FoxO genes that were also up-regulated at the transcriptional level (dos Santos et al. 2020). 
Table 4 Insulin-mimetic activity of ebselen, diphenyl diselenide, and $p$-chloro diphenyl diselenide in different experimental models

\begin{tabular}{|c|c|c|}
\hline & Experimental model & References \\
\hline \multirow[t]{5}{*}{ Ebselen } & Glucose-stimulated insulin secretion in murine islets & Wang et al. (2014) \\
\hline & Post stroke-induced hyperglycemia & Park et al. (2014) \\
\hline & Diazinon-induced hyperglycemia & Costa et al. (2012) \\
\hline & $\beta$-cell mass and function of Zucker diabetic fatty rats & Mahadevan et al. (2013) \\
\hline & $\begin{array}{l}\text { Metabolism and secretion of } \beta \text {-cells of Goto-Kakizaki non-obese diabetic } \\
\text { rats }\end{array}$ & Sasaki et al. (2013) \\
\hline \multirow[t]{5}{*}{ Diphenyl diselenide } & Acephate-induced hyperglycemia & Acker et al. (2014) \\
\hline & HepG2 and L6 myoblasts cells & da Rocha et al. (2013b) \\
\hline & Ovariectomy-induced metabolic disorders & da Rocha et al. (2011) \\
\hline & $\begin{array}{l}\text { Fructose and hydrochlorothiazide-induced metabolic } \\
\text { Disorders }\end{array}$ & Ribeiro et al. (2013) \\
\hline & Glucose-induced hyperglycemia in Zebrafish & dos Santos et al. (2020) \\
\hline$p$-Chloro & Feeding behavior & Bortolatto et al. (2015) \\
\hline \multirow[t]{2}{*}{ Diphenyl diselenide } & Monosodium glutamate-induced neuroendocrine obesity & $\begin{array}{l}\text { Quines et al. (2016a, 2017a } \\
\text {,2018) }\end{array}$ \\
\hline & High-fructose load-induced hyperglycemia & Quines et al. (2017b) \\
\hline
\end{tabular}

Moreover, both diphenyl diselenide and its $p$-chloro derivative, by inhibiting the hypothalamic 5-hydroxytryptamine (5HT) uptake, increase satiety suggesting their potential use as weight-reducing agents (Bortolatto et al. $2015)$. The $p$-chloro derivative satiating action was also reported to be most related to the lower orexin levels in the hypothalamus than the activation of thermogenesis in brown adipose tissue in rats (Bortolatto et al. 2017). From different experimental models (Quines et al. 2016a, 2017a,2018; b) emerged evidence that $p$-chloro derivative modulates glucose metabolism as well as reverses metabolic and mitochondrial dysfunction. Table 4 lists some experimental models in which ebselen, diphenyl diselenide, and its $p$-chloro derivative exert insulin-mimetic activity.

With regard to the effects of supplementary organoselenium compounds on glucose tolerance, selenomethionine was found to be effective in increasing pancreatic and hepatic RNA levels of GPx1 and glucose tolerance, even though it was unsuccessful in restoring insulin storage and secretion in nicotinamide/streptozotocin-induced mild diabetic mice (Ueno et al. 2014, 2018).

\section{Neuroprotective activity}

The history of ebselen borderline efficacy on brain ischemia and stroke clinical trials (Saito et al. 1998; Yamaguchi et al. 1998) was described by Parnham and Sies (2013), their commentaries, and personal perspectives reordered how the early research on this compound unwound.

With regard to the knowledge of the last 30 years, most derived from basic research that provided the full picture of ebselen neuroprotective effects in a great number of in vitro and in vivo models of brain toxicity. However, no attempt is made here to thoroughly discuss these reports; these have been adequately reviewed elsewhere (Hassan et al. 2016; Nogueira and Rocha 2011; Nogueira et al. 2004).

Since the pioneering study of Ünlü et al. (2002), the neuroprotective effects of ebselen on a spinal cord injury (SCI) model have been investigated. Applying the same dose (Aras et al. 2014; Jia et al. 2018; Kalayci et al. 2005) but an SCI model quite different from other studies, ebselen was proven to have a limited beneficial effect on the regeneration of the injury in the spinal cord white matter of rats (Ślusarczyk et al. 2019). Conversely, the improvement of motor function 14 days after the injury and the raise of anti-apoptotic and antioxidant markers had been previously reported (Jia et al. 2018; Kalayci et al. 2005). The authors suggest that these conflicting outcomes are explained by the fact that the neuroprotective effects of ebselen are not persistent long time after the administration (Ślusarczyk et al. 2019).

Glutamate, the main excitatory neurotransmitter in the mammalian central nervous system (CNS), in addition to have a large array of physiological functions, behaves as a potent neurotoxin in pathological conditions in which its metabolism is altered; therefore, neuronal death induced by glutamate is named excitotoxicity (Magi et al. 2019; Watkins and Jane 2006). Moreover, excitotoxicity has been implicated in different conditions, such as hypoxic/ischemic, traumatic brain injury, epilepsy, hypoglycemia, neuropsychiatric, and neurodegenerative disorders, among others (Olloquequi et al. 2018; Sharma et al. 2019; Zhang et al. 2020d), and organoselenium compounds have been investigated as potential therapeutic alternatives on this pathological phenomenon.

Diphenyl diselenide neuroprotective activity had been previously reported in the literature (Ghisleni et al. 2003, 
2008b; Machado et al. 2006; Nogueira et al. 2001; Posser et al. 2008) and, since that, propagated in experimental models of ischemia/reperfusion (Brüning et al. 2012a), stroke (Dobrachinski et al. 2014), auto-immune encephalomyelitis (Chanaday et al. 2011), mania (Brüning et al. 2012b), acetaminophen- (da Silva et al. 2012), chlorpyrifos- (Adedara et al. 2018), mycotoxin- (Baldissera et al. 2020c), manganese- (Adedara et al. 2016), and methyl-mercury- (Baldissera et al. 2020a; de Freitas et al. 2009; Glaser et al. 2013, 2014; Meinerz et al. 2011) induced neurotoxicity.

The modulation of the glutamatergic system, the antiinflammatory property, and antioxidant activity have been reported to play a role in diphenyl diselenide neuroprotective activity (Dalla Corte et al. 2012; Nogueira and Rocha 2010). In an attempt to explore the contribution of TrxR in neuroprotective effects of diselenides, diphenyl diselenide and its derivatives were investigated in the NADPH oxidation assay. The results revealed that diselenides were reduced to their selenol intermediates, indicating their role as substrates for cerebral TrxR (de Freitas and Rocha 2011). Consequently, any or all of these mechanisms of action could contribute to the neuroprotective action of diphenyl diselenide.

Glutaminase, an enzyme that catalyzes the hydrolysis of glutamine to glutamate, has been accounted for the generation of excitotoxic glutamate in the CNS and associated with ischemia, HIV-associated dementia, neurodegenerative diseases, and multiple sclerosis. Therefore, drug-like glutaminase inhibitors have been screened as potential neuroprotective agents. Ebselen was found as a potent inhibitor of glutaminase; however, due to the multiplicity of biological effects and lack of selectivity, Thomas and collaborators argue that it may be not a good prototype inhibitor for glutaminase inhibition in vivo (Thomas et al. 2013), which was confirmed by the Kosten study (Kosten et al. 2019).

In traumatic brain injury models, organoselenium compounds have opposite effects, whereas ebselen ( 3 to $30 \mathrm{mg} \mathrm{kg}^{-1}$, i.g) was neuroprotective, reducing nitric oxide levels and modulating the TLR4-mediated P38 MAPK signaling pathway (Wei et al. 2014), and diphenyl diselenide (10 and $25 \mathrm{mg} \mathrm{kg}^{-1}$, i.p) exacerbated post-concussive anxiogenic behavior and increased TNF $\alpha$ levels and longer telomeres (Yamakawa et al. 2020).

Given the fact that organoselenium compounds are effective in experimental models associated with the overproduction of ROS in the brain (Burger et al. 2006; Fachinetto et al. 2007; Souza et al. 2010) and antioxidant defenses are overwhelmed in sepsis (Cassol et al. 2010), diphenyl diselenide and ebselen have been investigated in a cecal ligation and perforation model of sepsis in rats (Silvestre et al. 2014). This study revealed that diphenyl diselenide $\left(50 \mathrm{mg} \mathrm{kg}^{-1}\right)$ reduced oxidative stress, mitochondrial dysfunction, and creatine kinase activity in cerebral structures of sepsis-survivor rats, whereas ebselen $\left(50 \mathrm{mg} \mathrm{kg}^{-1}\right)$ was reported to be less effective. The lower effectiveness of ebselen in this model was attributed to the reduced viability of rat hippocampal astrocytes via its action on the mitochondrial activity (Santofimia-Castano et al. 2013), whereas diphenyl diselenide positively modulated mitochondrial dysfunction (Dobrachinski et al. 2014) and reduced hepatic lipid peroxidation in a rat model of sepsis (Prauchner et al. 2011).

Although using different experimental models of Parkinson's disease, ebselen was reported to be ineffective against dopaminergic toxicity induced by 1-methyl-4-phenyl-1,2,3,6-tetrahydropyridine (MPTP) in the nigrostriatal tract of mice (Dhanasekaran et al. 2006), whereas diphenyl diselenide restored motor impairment, the decrease in ipsilateral striatal tyrosine hydroxylase levels, and mechanical and thermal nociception induced by 6-hydroxydopamine in rats (Da Rocha et al. 2013a, b; Sampaio et al. 2017b).

Regarding naturally occurring selenide-containing amino acids, we are only aware of very few published reports to date. In a culture of the hippocampal neurons, selenomethionine was effective against neuronal death induced by $\beta$-amyloid peptide (25-35) and $\mathrm{Fe}^{2+} / \mathrm{H}_{2} \mathrm{O}_{2}$ (Xiong et al. 2007), whereas selenocysteine antagonized oxygen-glucose deprivation-induced neurotoxicity (Wang et al. 2018b). The antioxidant potential of these amino acids was associated with their neuroprotective effects.

As shown by the massive indexed literature in the last decade, the effects of organoselenium compounds on memory have attracted the interest of the scientific community; therefore, the neuroprotective effects of these compounds on memory will be addressed separately in the next section.

\section{Memory-enhancing activity}

Cognitive decline and Alzheimer's dementia are conditions associated with lower levels of nonmetal element selenium (Loef et al. 2011; Cardoso et al. 2017; Rayman 2020; Reddy et al. 2017). An argument in favor of selenium supplementation in this condition (Rayman 2012) is that oxidative damage is a key component in the course of Alzheimer's disease (AD) (Butterfield and Halliwell 2019), even though the etiology and pathophysiology of this disease are still unknown.

In this way, experimental models are essential to understand the disease pathogenesis and to perform pre-clinical screening of novel therapies; however, often, they replicate only specific memory-associated cognitive impairments, which fail to resemble brain features of AD patients (Drummond and Wisniewski 2017). Research efforts in this field have uncovered ebselen (Table 5) as an inhibitor of acetylcholinesterase (AChE) activity (Luo et al. 2014; Martini et al. 2015; Mazzanti et al. 2009) and divalent metal transport (DMT1), which has been associated with the reduction of iron-induced tau hyperphosphorylation in human neuroblastoma SH-SY5Y cells (Xie et al. 2018a, 
Table 5 Effects of ebselen on end points of Alzheimer's disease experimental models

\begin{tabular}{|c|c|c|c|}
\hline End points & Effects & Experimental model & References \\
\hline \multirow[t]{2}{*}{ Acetylcholinesterase } & \multirow[t]{2}{*}{ Inhibits } & In vitro & $\begin{array}{l}\text { Luo et al. (2014); Mar- } \\
\text { tini et al. (2015) }\end{array}$ \\
\hline & & In vivo & Mazzanti et al. (2009) \\
\hline Divalent Metal Transport 1 & Inhibits & In vitro & Xie et al. $(2018 a, 2012)$ \\
\hline Amyloid precursor protein (APP) & Reduces & In vitro & Xie et al. (2018a) \\
\hline Ferrous iron-induced tau hyperphosphorylation & Reduces & In vitro & Xie et al. (2012) \\
\hline$\beta$-amyloid $(\mathrm{A} \beta)$ generation & Represses & In vitro & Xie et al. (2018a) \\
\hline BACE1 and presenilin & Inhibits & & \\
\hline Spatial and recognition memory & Improves & Sporadic & Martini et al. (2019) \\
\hline Apoptose, oxidative stress (hippocampus) & Reduces & $\mathrm{AD}$ & \\
\hline Apoptose, oxidative stress (cortex) & Reduces & $\begin{array}{l}\text { Sporadic } \\
\text { AD }\end{array}$ & Unsal et al. (2016) \\
\hline$\beta$-Amyloid and tau & Reduces & Triple-transgenic & Xie et al. (2017) \\
\hline Spatial memory & Improves & $\mathrm{AD}$ & \\
\hline Acethylcholinesterase & Inhibits & Scopolamine-induced Amnesia & Martini et al. (2018) \\
\hline Spatial recognition memory & Improves & & \\
\hline
\end{tabular}

$A D$ Alzheimer's disease

2012). Ebselen has been shown to improve memory and hallmarks of the disease in triple-transgenic (Xie et al. 2017) and sporadic AD models (Martini et al. 2019; Unsal et al. 2016), and scopolamine-induced amnesia, as well (Martini et al. 2018).

Several strategies have been employed to synthesize a series of ebselen derivatives aiming at achieving potential anti AD compounds; among them, we highlight the fusion of ebselen with donepezil and tacrine, potent AChE inhibitors used in clinical practice (Luo et al. 2013, 2014; Mao et al. 2013; Wang et al. 2016b). However, such structural changes have not been reported to provide significant therapeutic advantages.
Since the 2000s and the pioneering work of Rosa et al. (2003), diphenyl diselenide and its derivatives have been proven to be effective against memory impairment in rodent and non-rodent models (Jardim et al. 2017; Pinton et al. 2010; Souza et al. 2010; Stangherlin et al. 2008; Zamberlan et al. 2014; Zborowski et al. 2016). Some examples of experimental models are listed in Table 6, in which diphenyl diselenide-supplemented diet alone or in association with exercise or caffeine improved memory impairment in aged rats (Cechella et al. 2014a, b, 2018; Leite et al. 2014) and hypothyroidism condition (Dias et al. 2012); when administered by gavage, it was also effective in an ovariectomy model of menopause (da Rocha et al. 2012b).

Table 6 Effects of diphenyl diselenide and its derivatives in experimental models of memory impairment

\begin{tabular}{|c|c|c|c|c|}
\hline & Associated with & Experimental model & Effects & References \\
\hline \multirow[t]{5}{*}{ Diphenyl diselenide } & $\begin{array}{l}- \\
+ \text { Exercise }\end{array}$ & Middle-aged & $\uparrow \mathrm{STM}, \mathrm{LTM}$ & Cechella et al. (2014a) \\
\hline & $\begin{array}{l}- \\
+ \text { Caffeine }\end{array}$ & Middle-aged & $\begin{array}{l}\uparrow \mathrm{STM} \\
\uparrow \mathrm{LTM}\end{array}$ & Leite et al. (2014) \\
\hline & $\begin{array}{l}- \\
+ \text { Exercise }\end{array}$ & Old & $\uparrow$ STM, LTM, pCREB & Cechella et al. (2014b) \\
\hline & - & Hypothyroidism & $\uparrow \mathrm{MWM}$ & Dias et al. (2012) \\
\hline & - & Menopause & $\uparrow \mathrm{MWM}, \mathrm{AChE}$ & da Rocha et al. (2012b) \\
\hline p-Chloro-diphenyl diselenide & - & Old & $\uparrow \mathrm{LTM}$ & Bortolatto et al. (2012) \\
\hline$p$-Methoxyl-diphenyl diselenide & - & $\begin{array}{l}\text { Sporadic AD } \\
\text { A } \beta \text {-fragment 25-35 }\end{array}$ & $\uparrow \mathrm{MWM}, \mathrm{SDPA}, \mathrm{YM}$ & Pinton et al. $(2011,2013 \mathrm{a}, \mathrm{b})$ \\
\hline
\end{tabular}

old 24 months old, middle-aged 18 months old, LTM long-term memory, STM short-term memory, MWM Morris water maze, SDPA step-down passive avoidance, $Y M Y$ maze, $A C h E$ acetylcholinesterase activity 
In primary cultures of murine hippocampal neurons, diphenyl diselenide was reported to be tenfold more potent than ebselen against neurotoxicity induced by amyloid $A \beta$ (1-42), a factor associated with pathophysiological events of AD (Godoi et al. 2013).

Moreover, $p$-chloro diselenide derivative repeated administration enhanced memory and induced an antidepressantlike effect in old rats (Bortolatto et al. 2012). Regarding $p$-methoxyl derivative, this organoselenium compound supplemented in the diet or intragastrically administered was proven to be effective against sporadic AD models (Table 6) (Pinton et al. 2011, 2013a, b, c).

In particular, selenomethionine is the naturally occurring organoselenium most studied in models of AD (Attaran et al. 2020; Zhang et al. 2016a, 2018; Zheng et al. 2017a); its effects include increase of neurogenesis, reduction of $A \beta$, tau hyperphosphorylation, and neurofibrillary tangles formation, tau autophagic clearance, activation of glial cells, and regulation of metal dyshomeostasis, thereby improving learning and memory (Song et al. 2014; Xie et al. 2018b; Zhang et al. 2016b, 2017a, b; Zheng et al. 2019a).

A very recent study published by Zhang and collaborators proposed a novel mechanism by which selenomethionine improves cognitive impairment in AD. Using a selenoprotein $\mathrm{K}$ (SELENOK) knockout mice and AD model, the authors demonstrated that the decrease in SELENOK levels is associated with disequilibrium between synaptic and extrasynaptic NMDARs, suggesting that SELENOK is involved in the regulation of NMDARs and synaptic plasticity. The same study evaluated brain samples from AD patients and found reduced levels of SELENOK, but not of TrxR1, indicating a correlation between the decreased SELENOK level and AD. Selenomethionine restored synaptic deficits by modulating NMDARs and SELENOK in the brain of the AD mouse model (Zhang et al. 2020f). Table 7 summarizes the animal models of $\mathrm{AD}$ in which selenomethionine, diphenyl diselenide, and $p$-methoxyl-diphenyl diselenide were successfully investigated.

Table 7 Natural and synthetic organoselenium compounds tested in animal models of $\mathrm{AD}$

\begin{tabular}{|c|c|c|c|}
\hline & $\begin{array}{l}A \beta \text {-fragment } \\
25-35\end{array}$ & $\begin{array}{l}\text { Sporadic } \\
\mathrm{AD}^{\mathrm{a}}\end{array}$ & $\begin{array}{l}\text { Triple- } \\
\text { transgenic } \\
\mathrm{AD}^{\mathrm{b}}\end{array}$ \\
\hline Selenomethionine & & & ++ \\
\hline Ebselen & & ++ & ++ \\
\hline $\begin{array}{l}p \text {-Methoxyl- } \\
\text { diphenyl diselenide }\end{array}$ & ++ & ++ & \\
\hline
\end{tabular}

${ }^{\mathrm{a}}$ Icv streptozotocin-induced Sporadic AD, ${ }^{\mathrm{b}} \mathrm{APPswe}, \mathrm{PS} 1 \mathrm{M} 146 \mathrm{~V}$, and tauP301L

++ means effective
In terms of selenide effects on memory, we are only aware of a few published reports to date (Bortolatto et al. 2013b; Duarte et al. 2017; Peglow et al. 2017; Ramalho et al. 2018; Yan et al. 2019).

\section{Antioxidant activity}

The pioneering article of Muller and collaborators, demonstrating the GPx-like activity of ebselen (Möller et al. 1984), opened new avenues in the field of organoselenium applications and, since that, a multitude of synthetic entities with different chemical characteristics has been synthesized and their antioxidant properties investigated. As a result, major databases have been flooded with research articles related to antioxidant, GPx-like, radical-, and peroxynitrite-scavenging activities of newly developed molecules (Bortolatto et al. 2013a; Chagas et al. 2015; Fonseca et al. 2015; Ibrahim et al. 2012a, b, 2014a,2019; Junior et al. 2017; Luchese et al. 2012a; Peglow et al. 2017; Phadnis et al. 2014; Sauer et al. 2017; Singh et al. 2019; Stefanello et al. 2015a; Talas et al. 2015).

The GPx-like activity, the molecules' ability to emulate the reaction catalyzed by the native enzyme, has been viewed, although with some controversy (Barbosa et al. 2017; Wirth 2015), as the critical mechanism to design potential therapeutic agents, and it is undoubtedly the most studied property in the last decade (Ninomiya et al. 2011; Sarma and Mugesh 2008). In fact, several excellent reviews dealing with GPx-like activity have been published in the literature during recent years (Bhowmick and Mugesh 2015; Elsherbini et al. 2016; Orian and Toppo 2014; Sands et al. 2018).

The performance of cyclic and acyclic diorganyl selenides, and aryl and heteroaryl diselenides (Bhabak and Mugesh 2010; Sands et al. 2018) on the GPx-like assays has been investigated trying to overcome their low solubility in water, instability, and poor availability, which limit their therapeutic applications, and specially challenging to replicate in vivo conditions that require aqueous media and glutathione as the stoichiometric thiol (Sands et al. 2018). The studies have addressed synthetic approaches (Saeed et al. 2016), the merits of assays for measuring GPx-like activity, the nature of peroxides and thiols, the use of different solvents, the solubility of the reactants; their catalytic activities, redox mechanisms, and structure-activity relationships (Bhowmick and Mugesh 2018; Elsherbini et al. 2014; Hodage et al. 2012; Ibrahim et al. 2015; Jiang et al. 2015; Prabhu et al. 2012; Selvakumar et al. 2011; Singh et al. 2014, 2015; Thomas et al. 2012; Yu et al. 2018). The GPx-like activity of natural selenium-containing amino acids, selenocystine, methylselenocysteine, and selenomethionine has been also investigated and revealed selenocystine as the most efficient mimetic (Kumar et al. 2011). 
On the basis of selenium nutritional essentially for humans and other mammalian species (Rayman 2000, 2020), organic and inorganic sources have been used to the selenium supplementation in the animal industry and the organoselenium forms have been recognized to be more efficient in absorption, antioxidant activity, and tissue accumulation than inorganic selenium ones (Calvo et al. 2017; Shini et al. 2015).

Therefore, diphenyl diselenide was effective in enhancing serum basal antioxidant status (Table 8) and anti-inflammatory response in dairy sheep (Biazus et al. 2019), suggesting its nutraceutical application.

The selenium supplementation in the diet has been related with beneficial effects that increase the aquaculture potential of fish species (Zheng et al. 2018); therefore, diphenyl diselenide was effective to increase basal antioxidant tonus in tissues of silver catfish (Menezes et al. 2016). When nanocapsules were applied as carriers of diphenyl diselenide in the diet, an improvement in the muscle antioxidant capacity and growth performance of silver catfish were reported (Baldissera et al. 2020a, b, c, d) (Table 8).

The use of selenomethionine or its hydroxyl derivative supplemented in the diet has been also related with the enhancing in the antioxidant status in tissues of piglets and broilers (Chao et al. 2019; Falk et al. 2020; Wang et al. 2011b) (Table 8). A mechanistic proteomic study demonstrated that selenomethionine increases GPxs activities and down-regulates Rap1/MAPK/ERK signaling in chicken skeletal muscles (Liu et al. 2020b), highlighting the role of these pathways in inhibiting oxidative stress.

The antioxidant property of diphenyl diselenide ( $3 \mathrm{mg} \mathrm{kg}^{-1}, 60$ days) has been evaluated in models of oxidative stress induced by environmental concentrations of pesticides, atrazine (Marins et al. 2018), quinclorac (de Menezes et al. 2012), and clomazone (Menezes et al. 2013), in fishes.
As mentioned above, the GPx-like activity of organoselenium compounds has been not believed to be enough to justify their antioxidant properties in biological systems (Bartolini et al. 2015b; Wirth 2015). As a result, over the past few years, the mechanisms underlying organoselenium antioxidant effects have attracted interest. In 2015, Thomas and collaborators published an elegant study analyzing the charge density around $\mathrm{Se}-\mathrm{N}$ and $\mathrm{Se}-\mathrm{C}$ covalent bonds and the $\mathrm{Se} \cdots \mathrm{O}$ chalcogen-bonding modes in ebselen, which provides insights into the mechanism of drug action in this class of organoselenium antioxidants. This study revealed the optimized structures of ebselen with water and ROS, which would lead to the Se-N-bond cleavage (Thomas et al. 2015) (Scheme 3). The Se-N-bond cleavage is favored by the interaction between the nucleophile and the selenium atom, which weak the $\mathrm{Se}-\mathrm{N}$ bond.

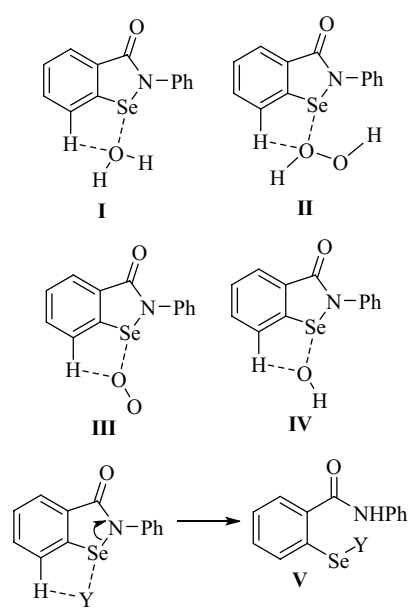

Scheme 3 Structures of ebselen intermediates with $\mathrm{H}_{2} \mathrm{O}$ (I), $\mathrm{H}_{2} \mathrm{O}_{2}$ (II), $\mathrm{O}_{2}{ }^{--}$(III), and $\mathrm{HO}^{*}$ (IV), and the general representation of ebselen Se-N-bond cleavage (V). Y represents the nucleophiles $\left(\mathrm{H}_{2} \mathrm{O}\right.$, $\mathrm{H}_{2} \mathrm{O}_{2}, \mathrm{O}_{2}{ }^{-}$, and $\mathrm{HO}^{\circ}$ )

Table 8 Effects of organoselenium compounds in basal antioxidant status

\begin{tabular}{|c|c|c|c|c|c|}
\hline & Supplementation & Species & Antioxidant markers & Other effects & References \\
\hline \multirow[t]{2}{*}{ Diphenyl diselenide } & $\begin{array}{l}3 \mu \mathrm{mol} \mathrm{kg}{ }^{-1} \text { (s.c) } \\
5 \text { doses }\end{array}$ & Sheep & $\begin{array}{l}\uparrow \mathrm{CAT}, \uparrow \mathrm{SOD}, \uparrow \mathrm{GST}, \uparrow \mathrm{GPx} \\
\downarrow \mathrm{ROS}, \downarrow \mathrm{LP}\end{array}$ & $\begin{array}{l}\uparrow \text { IL-10 } \\
\uparrow \text { milk fat }\end{array}$ & Biazus et al. (2019) \\
\hline & $\begin{array}{l}3 \mathrm{mg} \mathrm{kg}^{-1} \\
60 \text { days }\end{array}$ & Silver catfish & $\begin{array}{l}\uparrow \mathrm{AA}, \uparrow \mathrm{NPSH} \\
\downarrow \mathrm{ROS}, \downarrow \mathrm{PC}\end{array}$ & & Menezes et al. (2016) \\
\hline $\begin{array}{l}\text { Diphenyl diselenide nanocap- } \\
\text { sules }\end{array}$ & $\begin{array}{l}3 \mathrm{mg} \mathrm{kg}^{-1} \text { feed } \\
30 \text { days }\end{array}$ & Silver catfish & $\begin{array}{l}\uparrow \mathrm{CAT}, \uparrow \mathrm{SOD}, \uparrow \mathrm{ACAP}, \downarrow \mathrm{ROS}, \\
\quad \downarrow \mathrm{LP}\end{array}$ & $\uparrow[\mathrm{Se}]$ & $\begin{array}{l}\text { Baldissera et al. (2020d, } \\
\text { a, b, c) }\end{array}$ \\
\hline Selenomethionine & $\begin{array}{l}0.15 \mathrm{mg} \mathrm{kg}^{-1} \\
40 \text { days }\end{array}$ & Broilers & $\begin{array}{l}\uparrow \mathrm{GSH}, \uparrow \mathrm{SOD}, \uparrow \mathrm{GPx}, \\
\uparrow \mathrm{T}-\mathrm{AOC} \\
\downarrow \mathrm{MDA}\end{array}$ & $\begin{array}{l}\uparrow[\mathrm{Se}] \\
\uparrow \text { meat quality }\end{array}$ & Wang et al. (2011b) \\
\hline OH-selenomethionine & $\begin{array}{l}0.5 \mathrm{mg} \mathrm{kg}^{-1} \\
28 \text { days }\end{array}$ & Piglets & $\begin{array}{l}\uparrow \mathrm{GPx} \\
\downarrow \mathrm{MDA}\end{array}$ & $\uparrow[\mathrm{Se}]$ & Chao et al. (2019) \\
\hline
\end{tabular}

$C A T$ catalase, $S O D$ superoxide dismutase, GST glutathione $S$-transferase, GPx glutathione peroxidase, $R O S$ reactive oxygen species, $L P$ lipid peroxidation, $I L$ interleukin, $A C A P$ antioxidant capacity against peroxyl radicals, $P C$ protein carbonyl, $A A$ ascorbic acid, $T$ - $A O C$ total antioxidant capability, $M D A$ malondialdehyde, $G S H$ glutathione 
A molecular mechanism accounted for the organoselenium compounds antioxidant activity is the effect as "thiol modifier" by the activation of the Keap1/Nrf2 signaling pathway. In fact, increasing evidence has indicated that ebselen and diphenyl diselenide, by behaving as soft electrophiles, can oxidize critical cysteinyl residues in Keap1 that activates the Nrf2 signaling pathway and the subsequent transcription of antioxidant enzymes in cells (de Bem et al. 2013; dos Santos et al. 2020). This molecular mechanism of "adaptive stress response" activating the Nrf2 pathway, thereby leading to increased cellular protection, has also been demonstrated for other diselenide derivatives (Bartolini et al. 2015a).

\section{Conclusion}

Regarding its effects on mammalian cells, selenium is an element with two faces. Its deficiency is associated with an increased risk of diseases, including cancer, viral infection, and cardiovascular pathologies. In contrast, chronic exposure to dietary levels of selenium just above the ideal levels can increase the risk of cancer, neurodegenerative diseases, and type 2 diabetes. A great quantity of literature data has indicated a role for selenium in immunological and inflammatory responses; however, the exact molecular role played by selenium in these pathophysiological processes is still elusive. From the point of view of nutrition, the ideal selenium body burden is still unknown, but it seems evident that the ideal blood and body levels of selenium are narrow. Supplementation with either organic or inorganic forms of selenium should be implemented only after laboratory determination of deficient blood selenium levels. The supplementation should be discontinued or diminished as soon as the ideal level is attained. The narrow ideal levels of selenium ingestion are related to its unique reactivity in the physiological milieu. Metabolically, inorganic selenium (e.g., selenite or $\mathrm{Se}^{+4}$ and selenate or $\mathrm{Se}^{+6}$ ) is metabolized to selenide $\left(\mathrm{HSe}^{-}\right)$, which can be incorporated in selenoproteins as selenocysteinyl residues. An excess of selenide can generate reactive and toxic metabolites that can deregulate cell physiology via disruption of thiol-containing proteins (for instance, methylselenyl radical). Our knowledge on how an excess of selenium ingestion interferes with the normal physiology of the 25 human selenoproteins is still very limited. In contrast to inorganic selenium forms, the metabolism of organic forms of selenium will depend greatly on their chemical structure. For instance, selenomethionine can be metabolized to methylselenol, selenocysteine, and selenide (Fig. 4), but an excess of selenomethionine can also generate reactive intermediates (Fig. 4) and even cause death in animals and humans. Other naturally occurring selenium compounds (methylselenocysteine, selenocystine, etc.)

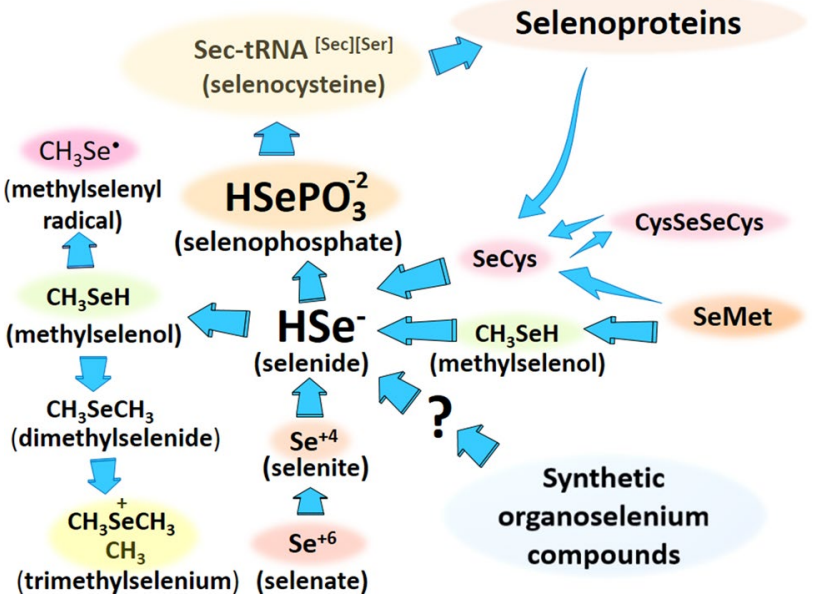

Fig. 4 Main metabolites of common dietary inorganic selenium forms (selenite or $\mathrm{Se}^{4+}$ and selenate or $\mathrm{Se}^{6+}$ ) and from selenomethionine (SeMet) to selenide $\left(\mathrm{HSe}^{-}\right)$. Selenide is the key intermediate in the incorporation of selenium into the organic moiety of serine forming the selenocysteinyl residue at the level of the specific t-RNA (Sec-t-RNA[Sec][Ser]]) that will be incorporated in the structure of selenoproteins. Selenomethionine (a naturally occurring organic form of selenium) can be metabolized directly to methylselenol or via the transulfuration pathway to a minor amount of selenocysteine (SeCys) (Burk and Hill 2015), which due to its reactivity is expected to be oxidized to selenocystine (CysSeSeCys). The methylselenol can be demethylated to selenide, and SeCys and CysSeSeCys can also be metabolized to selenide. The methylselenol intermediate can be further methylated to dimethylselenide (which can be excreted via inhalation) and trimethylselenium cation (which is excreted in the urine). The methylselenol can also be oxidized to dimethyldiselenide or can form the extreme reactive selenyl radical. The elusive metabolism of synthetic organoselenium compounds into the inorganic selenide pool is indicated by a quotation mark

can also be metabolized to inorganic selenium, but some synthetic organoselenium compounds (for instance, ebselen) do not release the selenium from its organic moiety. Differences in the metabolism of synthetic organoselenium compounds can have nutritional and pharmacological consequences and we have little knowledge about the metabolism of the majority of synthetic selenium-containing organocompounds. Another point that has not been studied is the potential metabolism of organoselenium compounds by intestinal microorganisms (Takahashi et al. 2020). This is of particular importance, because the oral route has been shown to be a potential route to administer pharmacological active selenium compounds (Nogueira and Rocha 2011). Indeed, we do not know which of them can contribute to the inorganic pool of selenium in vivo (either directly or after metabolic changes in the gastrointestinal tract) (Fig. 4). It is important to emphasize that organic forms of selenium will contribute to selenoprotein synthesis only when they can be metabolized to selenide (Fig. 4). 
The remarkable interest in organic molecule-containing selenium was reinvigorated in the late 1970s when ebselen (a molecule originally synthesized in 1924) was shown to be a mimetic of GPx and an antioxidant. Contrary to the expectations of the researchers, ebselen was not a source of selenium for GPx biosynthesis, but it was an interesting compound with pharmacological potential both as an antiinflammatory and antioxidant agent in a variety of in vitro and in vivo models of diseases (Parnham and Sies 2013). In the late 1990s, ebselen was tested in 3 human clinical trials to treat brain ischemia and stroke with a borderline efficacy. However, its efficacy was not considered sufficient to justify its clinical use in brain ischemia and related pathologies. Despite the clinical failures of ebselen, it was proven to be a safe drug for human use. To date, there are approved and ongoing clinical trials with ebselen to treat bipolar disorder and moderate and severe COVID-19. Here, in this review, we have discussed some organoselenium compounds with potential pharmacological applications, including some ebselen derivatives and several diselenides. Although ebselen and diselenides can have some overlapping pharmacological properties, their molecular targets are not identical. However, they have similar anti-inflammatory and antioxidant activities, possibly, via activation of transcription factors regulating the expression of antioxidant genes. In short, our knowledge about the pharmacological properties of simple organoselenium compounds is still elusive. However, contrary to our early expectations that they could imitate selenoproteins, organoselenium compounds seem to have non-specific modulatory activation of antioxidant pathways and specific inhibitory effects in some thiol-containing proteins. For instance, the inhibition of 5'-IMPase (an enzyme that is a target of lithium) via oxidation of a critical cysteinyl residue in the protein is thought to be involved in the antidepressive effects of ebselen (Singh et al. 2013). Of potential significance for the treatment of SARS-CoV-2 infection, ebselen has been shown to inhibit the two thiol-containing virus proteases (the main protease and the papain-like protease) and to decrease the virus replication in vitro (Jin et al. 2020; Sies and Parnham 2020). The thiol-oxidizing properties of organoselenium compounds have been considered the molecular basis of their chronic toxicity; however, the acute use of organoselenium compounds as inhibitors of specific thiol-containing enzymes can be of therapeutic significance. In summary, the outcomes of the clinical trials of ebselen as a mimetic of lithium or as an inhibitor of SARS-CoV-2 proteases will be important to the field of organoselenium compounds synthesis. In addition, the development of computational techniques that could predict rational modifications in the structure of ebselen and other organoselenium compounds to increase their specificity is also required to construct a library of thiol-modifying agents with selectivity toward specific target proteins.
Acknowledgements The authors wish to sincerely thank their coworkers listed in the references for their dedicated collaboration and devotion. We gratefully acknowledge Universidade Federal de Santa Maria (UFSM), Coordenação de Aperfeiçoamento de Pessoal de Nível Superior (CAPES, PROEX: 23038.005450/2020-19), Conselho Nacional de Desenvolvimento Científico e Tecnológico (CNPq), and FAPERGS for the financial support. C.W.N, J.B.T.R., and N.B.B.V. are recipient of CNPq scientific productivity fellowships.

\section{References}

AbdelKhalek A, Abutaleb NS, Mohammad H, Seleem MN (2018) Repurposing ebselen for decolonization of vancomycin-resistant enterococci (VRE). PLoS ONE 13(6):e0199710

Acker CI, Nogueira CW (2014) Diphenyl diselenide protects against metabolic disorders induced by acephate acute exposure in rats. Environ Toxicol 29(6):665-671

Acker CI, Luchese C, Prigol M, Nogueira CW (2009) Antidepressant-like effect of diphenyl diselenide on rats exposed to malathion: involvement of $\mathrm{Na}+\mathrm{K}+$ ATPase activity. Neurosci Lett 455(3): 168-172

Acker CI, Souza ACG, dos Santos MP, Mazzanti CM, Nogueira CW (2012) Diphenyl diselenide attenuates hepatic and hematologic toxicity induced by chlorpyrifos acute exposure in rats. Environ Sci Pollut Res 19(8):3481-3490

Adani G, Filippini T, Garuti C et al (2020a) Environmental risk factors for early-onset Alzheimer's dementia and frontotemporal dementia: a case-control study in northern Italy. Int J Environ Res Public Health 17(21):7941

Adani G, Filippini T, Michalke B, Vinceti M (2020b) Selenium and other trace elements in the etiology of Parkinson's disease: a systematic review and meta-analysis of case-control studies. Neuroepidemiology 54(1):1-23

Addinsall AB, Wright CR, Andrikopoulos S, van der Poel C, Stupka N (2018) Emerging roles of endoplasmic reticulum-resident selenoproteins in the regulation of cellular stress responses and the implications for metabolic disease. Biochem J 475(6):1037-1057

Adedara IA, Abolaji AO, Rocha JB, Farombi EO (2016) Diphenyl diselenide protects against mortality, locomotor deficits and oxidative stress in Drosophila melanogaster model of manganeseinduced neurotoxicity. Neurochem Res 41(6):1430-1438

Adedara IA, Owoeye O, Awogbindin IO, Ajayi BO, Rocha JB, Farombi EO (2018) Diphenyl diselenide abrogates brain oxidative injury and neurobehavioural deficits associated with pesticide chlorpyrifos exposure in rats. Chem Biol Interact 296:105-116

Aggarwal R, Gathwala G, Yadav S, Kumar P (2016) Selenium supplementation for prevention of late-onset sepsis in very low birth weight preterm neonates. J Trop Pediatr 62(3):185-193

Al-Brakati A, Kassab R, Lokman M, Elmahallawy E, Amin H, Abdel Moneim A (2019) Role of thymoquinone and ebselen in the prevention of sodium arsenite-induced nephrotoxicity in female rats. Hum Exp Toxicol 38(4):482-493

Alhazzani W, Jacobi J, Sindi A et al (2013) The effect of selenium therapy on mortality in patients with sepsis syndrome: a systematic review and meta-analysis of randomized controlled trials. Crit Care Med 41(6):1555-1564

Allingstrup M, Afshari A (2015) Selenium supplementation for critically ill adults. Cochrane Database Syst Rev 27(7):1-57

Álvarez-Pérez M, Ali W, Marć MA, Handzlik J, Domínguez-Álvarez E (2018) Selenides and diselenides: a review of their anti-cancer and chemopreventive activity. Molecules 23(3):628

Amaral B, Cargnelutti J, Mortari A et al (2020) Diphenyl diselenide and cidofovir present anti-viral activity against Bovine Alphaherpesvirus 2 in vitro and in a sheep model. Res Vet Sci 134:78-85 
Antoniadou I, Kouskou M, Arsiwala T et al (2018) Ebselen has lithiumlike effects on central 5-HT2A receptor function. Br J Pharmacol 175(13):2599-2610

Aras M, Altaş M, Meydan S et al (2014) Effects of ebselen on ischemia/ reperfusion injury in rat brain. Int J Neurosci 124(10):771-776

Araujo PCO, Sari MHM, Jardim NS, Jung JTK, Brüning CA (2020) Effect of m-trifluoromethyl-diphenyl diselenide on acute and subchronic animal models of inflammatory pain: behavioral, biochemical and molecular insights. Chem Biol Interact 317:108941

Arnold M, Forte J, Osterberg J, Di Giulio R (2016) Antioxidant rescue of selenomethionine-induced teratogenesis in zebrafish embryos. Arch Environ Contam Toxicol 70(2):311-320

Aruoma OI (1997) Scavenging of hypochlorous acid by carvedilol and ebselen in vitro. Gen Pharmacol 28(2):269-272

Attaran A, Salahinejad A, Naderi M, Crane AL, Niyogi S, Chivers DP (2020) Effects of chronic exposure to selenomethionine on social learning outcomes in zebrafish (Danio rerio): serotonergic dysregulation and oxidative stress in the brain. Chemosphere 247:125898

Avery JC, Hoffmann PR (2018) Selenium, selenoproteins, and immunity. Nutrients 10(9):1203

Baek JM, Kim J-Y, Yoon K-H, Oh J, Lee MS (2016) Ebselen is a potential anti-osteoporosis agent by suppressing receptor activator of nuclear factor kappa-B ligand-induced osteoclast differentiation in vitro and lipopolysaccharide-induced inflammatory bone destruction in vivo. Int J Biol Sci 12(5):478

Baldissera MD, Souza CF, Alessio KO et al (2020d) Diphenyl diselenide-loaded nanocapsules in silver catfish feed enhance growth, improve muscle antioxidant/oxidant status and increase selenium deposition: advantages of nanotechnology for fish health. Aquac Res 51(10):4196-4205

Baldissera MD, Souza CF, da Silva AS, Henn AS, Flores EM, Baldisserotto B (2020a) Diphenyl diselenide dietary supplementation alleviates behavior impairment and brain damage in grass carp (Ctenopharyngodon idella) exposed to methylmercury chloride. Comp Biochem Physiol C Toxicol Pharmacol 229:108674

Baldissera MD, Souza CF, da Silva HNP et al (2020b) Diphenyl diselenide modulates splenic purinergic signaling in silver catfish fed diets contaminated with fumonisin B1: An attempt to improve immune and hemostatic responses. Comp Biochem Physiol C Toxicol Pharmacol 227:108624

Baldissera MD, Souza CF, da Silva HNP et al (2020c) Diphenyl diselenide dietary supplementation protects against fumonisin B1-induced oxidative stress in brains of the silver catfish Rhamdia quelen. Comp Biochem Physiol C Toxicol Pharmacol 231:108738

Bampi SR, Casaril AM, Sousa FSS et al (2019) Repeated administration of a selenium-containing indolyl compound attenuates behavioural alterations by streptozotocin through modulation of oxidative stress in mice. Pharmacol Biochem Behav 183:46-55

Bampi SR, Casaril AM, Domingues M et al (2020a) Depression-like behavior, hyperglycemia, oxidative stress, and neuroinflammation presented in diabetic mice are reversed by the administration of 1-methyl-3-(phenylselanyl)-1H-indole. J Psychiatr Res 120:91-102

Bampi SR, Casaril AM, Fronza MG et al (2020b) The selenocompound 1-methyl-3-(phenylselanyl)-1H-indole attenuates depression-like behavior, oxidative stress, and neuroinflammation in streptozotocin-treated mice. Brain Res Bull 161:158-165

Barbosa N, Rocha J, Zeni G, Emanuelli T, Beque MM, Braga A (1998) Effect of organic forms of selenium on $\delta$-aminolevulinate dehydratase from liver, kidney, and brain of adult rats. Toxicol Appl Pharmacol 149(2):243-253

Barbosa N, Rocha J, Wondracek D, Perottoni J, Zeni G, Nogueira C (2006) Diphenyl diselenide reduces temporarily hyperglycemia: possible relationship with oxidative stress. Chem Biol Interact 163(3):230-238

Barbosa N, Rocha J, Soares J et al (2008) Dietary diphenyl diselenide reduces the STZ-induced toxicity. Food Chem Toxicol 46(1):186-194

Barbosa CF, Tonin AA, Da Silva AS et al (2014) Diphenyl diselenide and sodium selenite associated with chemotherapy in experimental toxoplasmosis: influence on oxidant/antioxidant biomarkers and cytokine modulation. Parasitology 141(13):1761

Barbosa NV, Nogueira CW, Nogara PA, Andreza F, Aschner M, Rocha JB (2017) Organoselenium compounds as mimics of selenoproteins and thiol modifier agents. Metallomics 9(12):1703-1734

Barkus C, Ferland J-MN, Adams WK et al (2018) The putative lithiummimetic ebselen reduces impulsivity in rodent models. J Psychopharmacol 32(9):1018-1026

Bartolini D, Commodi J, Piroddi M et al (2015a) Glutathione $S$-transferase pi expression regulates the Nrf2-dependent response to hormetic diselenides. Free Radic Biol Med 88:466-480

Bartolini D, Piroddi M, Tidei C et al (2015b) Reaction kinetics and targeting to cellular glutathione $\mathrm{S}$-transferase of the glutathione peroxidase mimetic $\mathrm{PhSeZnCl}$ and its D,L-polylactide microparticle formulation. Free Radic Biol Med 78:56-65

Basarslan F, Yilmaz N, Davarci I et al (2013) Effects of ebselen on radiocontrast media-induced hepatotoxicity in rats. Toxicol Ind Health 29(8):746-752

Bastola MM, Locatis C, Maisiak R, Fontelo P (2020) Selenium, copper, zinc and hypertension: an analysis of the National Health and Nutrition Examination Survey (2011-2016). BMC Cardiovasc Disord 20(1):1-8

Beckett GJ, Arthur JR (2005) Selenium and endocrine systems. J Endocrinol 184(3):455-465

Beckman JA, Goldfine AB, Leopold JA, Creager MA (2016) Ebselen does not improve oxidative stress and vascular function in patients with diabetes: a randomized, crossover trial. Am J Physiol Heart Circ Physiol 311(6):H1431-H1436

Beil W, Staar U, Sewing K-F (1990) Interaction of the anti-inflammatory seleno-organic compound ebselen with acid secretion in isolated parietal cells and gastric $\mathrm{H}+/ \mathrm{K}+-\mathrm{ATPase}$. Biochem Pharmacol 40(9): 1997-2003

Besckow EM, Nonemacher NT, Garcia CS et al (2020) Antidepressant-like effect of a selenopropargylic benzamide in mice: involvement of the serotonergic system. Psychopharmacology 237(10):3149-3159

Bhabak KP, Mugesh G (2010) Functional mimics of glutathione peroxidase: bioinspired synthetic antioxidants. Acc Chem Res 43(11):1408-1419

Bhattacharya A (2011) Methylselenocysteine-a promising antiangiogenic agent for overcoming drug delivery barriers in solid malignancies for therapeutic synergy with anti-cancer drugs. Expert Opin Drug Deliv 8(6):749-763

Bhave G, Gereau RW (2004) Posttranslational mechanisms of peripheral sensitization. J Neurobiol 61(1):88-106

Bhowmick D, Mugesh G (2015) Insights into the catalytic mechanism of synthetic glutathione peroxidase mimetics. Org Biomol Chem 13(41):10262-10272

Bhowmick D, Mugesh G (2018) Antioxidant activity of amine-based organoselenium compounds having Intramolecular Se-Se bonds. Indian J Heterocycl Chem 28(1):43-50

Biazus AH, Cazarotto CJ, Machado G et al (2019) Diphenyl diselenide subcutaneous supplementation of dairy sheep: effects on oxidant and antioxidant status, inflammatory response and milk composition. Anim Prod Sci 59(3):461-470

Bijian K, Zhang Z, Xu B et al (2012) Synthesis and biological activity of novel organoselenium derivatives targeting multiple kinases 
and capable of inhibiting cancer progression to metastases. Eur J Med Chem 48:143-152

Birmann PT, Sousa FS, de Oliveira DH et al (2018) 3-(4-Chlorophenylselanyl)-1-methyl-1H-indole, a new selenium compound elicits an antinociceptive and anti-inflammatory effect in mice. Eur J Pharmacol 827:71-79

Bleys J, Navas-Acien A, Guallar E (2008) Serum selenium levels and all-cause, cancer, and cardiovascular mortality among US adults. Arch Intern Med 168(4):404-410

Bloos F, Trips E, Nierhaus A et al (2016) Effect of sodium selenite administration and procalcitonin-guided therapy on mortality in patients with severe sepsis or septic shock: a randomized clinical trial. JAMA Intern Med 176(9):1266-1276

Borges LP, Borges VC, Moro AV, Nogueira CW, Rocha JBT, Zeni G (2005) Protective effect of diphenyl diselenide on acute liver damage induced by 2-nitropropane in rats. Toxicology 210(1): $1-8$

Borges LP, Nogueira CW, Panatieri RB, Rocha JBT, Zeni G (2006) Acute liver damage induced by 2-nitropropane in rats: effect of diphenyl diselenide on antioxidant defenses. Chem Biol Interact 160(2):99-107

Borges LP, Brandao R, Godoi B, Nogueira CW, Zeni G (2008) Oral administration of diphenyl diselenide protects against cadmium-induced liver damage in rats. Chem Biol Interact 171(1):15-25

Bortolatto CF, Wilhelm EA, Chagas PM, Nogueira CW (2012) $p$-Chloro-diphenyl diselenide, an organoselenium compound, with antidepressant-like and memory enhancer actions in aging male rats. Biogerontology 13(3):237-249

Bortolatto CF, Chagas PM, Wilhelm EA, Zeni G, Nogueira CW (2013a) 2, 2'-dithienyl diselenide, an organoselenium compound, elicits antioxidant action and inhibits monoamine oxidase activity in vitro. J Enzyme Inhib Med Chem 28(4):677-684

Bortolatto CF, Souza ACG, Wilhelm EA, Nogueira CW (2013b) Acute treatment with bis selenide, an organic compound containing the trace element selenium, prevents memory deficits induced by reserpine in rats. Biol Trace Elem Res 151(1):92-99

Bortolatto CF, Wilhelm EA, Roman SS, Nogueira CW (2014) (E)2-Benzylidene-4-phenyl-1, 3-diselenole ameliorates signals of renal injury induced by cisplatin in rats. J Appl Toxicol 34(1):87-94

Bortolatto CF, Heck SO, Gai BM, Zborowski VA, Neto JS, Nogueira CW (2015) Effects of diphenyl and p-chloro-diphenyl diselenides on feeding behavior of rats. Psychopharmacology 232(13):2239-2249

Bortolatto CF, Nogueira CW, Porteiro B, Imbernón M, Nogueiras R (2017) Hypothalamic pathways regulate the anorectic action of $p$-chloro-diphenyl diselenide in rats. Eur J Pharmacol 815:241-250

Brandão R, Borges LP, Oliveira Rd, Rocha JB, Nogueira CW (2008) Diphenyl diselenide protects against hematological and immunological alterations induced by mercury in mice. J Biochem Mol Toxicol 22(5):311-319

Brandão R, Moresco RN, Bellé LP et al (2011) Diphenyl diselenide potentiates nephrotoxicity induced by mercuric chloride in mice. J Appl Toxicol 31(8):773-782

Brandao R, Acker CI, Leite MR, Barbosa NB, Nogueira CW (2009) Diphenyl diselenide protects against glycerol-induced renal damage in rats. J Appl Toxicol 29(7):612-618

Brito VB, Folmer V, Puntel GO et al (2006) Diphenyl diselenide and 2, 3-dimercaptopropanol increase the PTZ-induced chemical seizure and mortality in mice. Brain Res Bull 68(6):414-418

Brod LMP, Fronza MG, Vargas JP et al (2016) Involvement of monoaminergic system in the antidepressant-like effect of (octylseleno)-xylofuranoside in the mouse tail suspension test. Prog Neuropsychopharmacol Biol Psychiatry 65:201-207
Brodin O, Eksborg S, Wallenberg M et al (2015) Pharmacokinetics and toxicity of sodium selenite in the treatment of patients with carcinoma in a phase I clinical trial: the SECAR study. Nutrients 7(6):4978-4994

Brüne B, Diewald B, Ullrich V (1991) Ebselen affects calcium homeostasis in human platelets. Biochem Pharmacol 41(12):1805-1811

Brüning CA, Prigol M, Roehrs JA, Zeni G, Nogueira CW (2010) Evidence for the involvement of $\mu$-opioid and $\delta$-opioid receptors in the antinociceptive effect caused by oral administration of $\mathrm{m}$-trifluoromethyl-diphenyl diselenide in mice. Behav Pharmacol 21(7):621-626

Brüning CA, Souza ACG, Gai BM, Zeni G, Nogueira CW (2011) Antidepressant-like effect of $\mathrm{m}$-trifluoromethyl-diphenyl diselenide in the mouse forced swimming test involves opioid and serotonergic systems. Eur J Pharmacol 658(2-3):145-149

Brüning CA, Prigol M, Luchese C, Pinton S, Nogueira CW (2012b) Diphenyl diselenide ameliorates behavioral and oxidative parameters in an animal model of mania induced by ouabain. Prog Neuropsychopharmacol Biol Psychiatry 38(2):168-174

Brüning CA, Prigol M, Luchese C et al (2012a) Protective effect of diphenyl diselenide on ischemia and reperfusion-induced cerebral injury: involvement of oxidative stress and pro-inflammatory cytokines. Neurochem Res 37(10):2249-2258

Brüning CA, Gai BM, Soares SM, Martini F, Nogueira CW (2014) Serotonergic systems are implicated in antinociceptive effect of $\mathrm{m}$-trifluoromethyl diphenyl diselenide in the mouse glutamate test. Pharmacol Biochem Behav 125:15-20

Brüning CA, Martini F, Soares SM et al (2015a) m-Trifluoromethyldiphenyl diselenide, a multi-target selenium compound, prevented mechanical allodynia and depressive-like behavior in a mouse comorbid pain and depression model. Prog Neuropsychopharmacol Biol Psychiatry 63:35-46

Brüning CA, Martini F, Soares SM, Savegnago L, Sampaio TB, Nogueira CW (2015b) Depressive-like behavior induced by tumor necrosis factor- $\alpha$ is attenuated by $\mathrm{m}$-trifluoromethyl-diphenyl diselenide in mice. J Psychiatr Res 66:75-83

Brzački V, Mladenović B, Dimić D et al (2019) Comparison between the effects of selenomethionine and $S$-adenosylmethionine in preventing cholestasis-induced rat liver damage. Amino Acids 51(5):795-803

Bubolz AH, Wu Q, Larsen BT, Gutterman DD, Liu Y (2007) Ebselen reduces nitration and restores voltage-gated potassium channel function in small coronary arteries of diabetic rats. Am J Physiol Heart Circ Physiol 293(4):H2231-H2237

Bueno D, Meinerz D, Waczuk E, de Souza D, Batista Rocha J (2018) Toxicity of organochalcogens in human leukocytes is associated, but not directly related with reactive species production, apoptosis and changes in antioxidant gene expression. Free Radic Res 52(10): 1158-1169

Bueno Rosseti I, Taube Junior P, Ladeira B, de Campos C, Teixeira B, da Rocha J, Silva Costa M (2014) Biofilm formation by Candida albicans is inhibited by 4, 4-dichloro diphenyl diselenide (p-ClPhSe) 2. Curr Drug Discov Technol 11(3):234-238

Bulteau A-L, Chavatte L (2015) Update on selenoprotein biosynthesis. Antioxid Redox Signal 23(10):775-794

Burger ME, Fachinetto R, Wagner C et al (2006) Effects of diphenyldiselenide on orofacial dyskinesia model in rats. Brain Res Bull 70(2): $165-170$

Burk RF, Hill KE (2015) Regulation of selenium metabolism and transport. Annu Rev Nutr 35:109-134

Butterfield DA, Halliwell B (2019) Oxidative stress, dysfunctional glucose metabolism and Alzheimer disease. Nat Rev Neurosci 20(3):148-160

Caeran Bueno D, Meinerz DF, Allebrandt J et al (2013) Cytotoxicity and genotoxicity evaluation of organochalcogens in human leucocytes: a comparative study between ebselen, diphenyl 
diselenide, and diphenyl ditelluride. BioMed Res Int. https:// doi.org/10.1155/2013/537279

Calvo L, Toldrá F, Rodríguez AI, López-Bote C, Rey AI (2017) Effect of dietary selenium source (organic vs. mineral) and muscle $\mathrm{pH}$ on meat quality characteristics of pigs. Food Sci Nutr 5(1):94-102

Cao S, Durrani FA, Rustum YM (2004) Selective modulation of the therapeutic efficacy of anti-cancer drugs by selenium containing compounds against human tumor xenografts. Clin Cancer Res 10(7):2561-2569

Cao S, Durrani F, Toth K, Rustum Y (2014) Se-methylselenocysteine offers selective protection against toxicity and potentiates the antitumour activity of anti-cancer drugs in preclinical animal models. Br J Cancer 110(7):1733-1743

Cardoso Br R, Hare DJ, Lind M et al (2017) The apoe $\varepsilon 4$ allele is associated with lower selenium levels in the brain: implications for Alzheimer's disease. ACS Chem Neurosci 8(7):1459-1464

Carvalho NR, da Rosa EF, da Silva MH et al (2013) New therapeutic approach: diphenyl diselenide reduces mitochondrial dysfunction in acetaminophen-induced acute liver failure. PLoS ONE 8(12):e81961

Carvalho NR, Tassi CC, Dobraschinski F et al (2017) Reversal of bioenergetics dysfunction by diphenyl diselenide is critical to protection against the acetaminophen-induced acute liver failure. Life Sci 180:42-50

Casaril AM, Domingues M, Fronza M et al (2017) Antidepressantlike effect of a new selenium-containing compound is accompanied by a reduction of neuroinflammation and oxidative stress in lipopolysaccharide-challenged mice. J Psychopharmacol 31(9):1263-1273

Casaril AM, Domingues M, Bampi SR et al (2019a) The seleniumcontaining compound 3-((4-chlorophenyl) selanyl)-1-methyl-1H-indole reverses depressive-like behavior induced by acute restraint stress in mice: modulation of oxido-nitrosative stress and inflammatory pathway. Psychopharmacology 236(10):2867-2880

Casaril AM, Domingues M, de Andrade LD et al (2019b) Depressionand anxiogenic-like behaviors induced by lipopolysaccharide in mice are reversed by a selenium-containing indolyl compound: behavioral, neurochemical and computational insights involving the serotonergic system. J Psychiatr Res 115:1-12

Cassol OJ, Rezin GT, Petronilho FC et al (2010) Effects of $N$-acetylcysteine/deferoxamine, taurine and RC-3095 on respiratory chain complexes and creatine kinase activities in rat brain after sepsis. Neurochem Res 35(4):515-521

Cechella JL, Leite MR, Gai RM, Zeni G (2014a) The impact of a diphenyl diselenide-supplemented diet and aerobic exercise on memory of middle-aged rats. Physiol Behav 135:125-129

Cechella JL, Leite MR, Rosario AR, Sampaio TB, Zeni G (2014b) Diphenyl diselenide-supplemented diet and swimming exercise enhance novel object recognition memory in old rats. Age 36(4):9666

Cechella JL, Leite MR, Pinton S, Zeni G, Nogueira CW (2018) Neuroprotective benefits of aerobic exercise and organoselenium dietary supplementation in hippocampus of old rats. Mol Neurobiol 55(5):3832-3840

Chagas PM, Bortolatto CF, Wilhelm EA, Nogueira CW (2013a) High doses of 2,2'-dithienyl diselenide cause systemic toxicity in rats: an in vitro and in vivo study. J Appl Toxicol 33(6):480-487

Chagas PM, Bortolatto CF, Wilhelm EA, Roehrs JA, Nogueira CW (2013b) Bis (phenylimidazoselenazolyl) diselenide: a compound with antinociceptive properties in mice. Behav Pharmacol 24(1):37-44

Chagas PM, Rosa SG, Sari MHM et al (2014) Evaluation of the pharmacological properties of salicylic acid-derivative organoselenium: 2-hydroxy-5-selenocyanatobenzoic acid as an anti-inflammatory and antinociceptive compound. Pharmacol Biochem Behav 118:87-95

Chagas PM, Fulco BdCW, Pesarico AP, Roehrs JA, Nogueira CW (2015) Bis (phenylimidazoselenazolyl) diselenide as an antioxidant compound: an in vitro and in vivo study. Chem Biol Interact 233:14-24

Chagas PM, Fulco BC, Sari MH, Roehrs JA, Nogueira CW (2017a) Bis (phenylimidazoselenazolyl) diselenide elicits antinociceptive effect by modulating myeloperoxidase activity, NOx and NFkB levels in the collagen-induced arthritis mouse model. J Pharm Pharmacol 69(8):1022-1032

Chagas PM, Fulco BdCW, Pesarico AP, Roehrs JA, Nogueira CW (2017b) Effectiveness of bis (phenylimidazoselenazolyl) diselenide on a mouse model of inflammatory nociception. Biomed Pharmacother 96:56-63

Chakraborty P, Roy SS, Basu A, Bhattacharya S (2016) Sensitization of cancer cells to cyclophosphamide therapy by an organoselenium compound through ROS-mediated apoptosis. Biomed Pharmacother 84:1992-1999

Chanaday NL, Andreza F, Roth GA (2011) Effect of diphenyl diselenide on the development of experimental autoimmune encephalomyelitis. Neurochem Int 59(8):1155-1162

Chander PN, Gealekman O, Brodsky SV et al (2004) Nephropathy in Zucker diabetic fat rat is associated with oxidative and nitrosative stress: prevention by chronic therapy with a peroxynitrite scavenger ebselen. J Am Soc Nephrol 15(9):2391-2403

Chao Y, Yu B, He J et al (2019) Effects of different levels of dietary hydroxy-analogue of selenomethionine on growth performance, selenium deposition and antioxidant status of weaned piglets. Arch Anim Nutr 73(5):374-383

Chapelle N, Martel M, Toes-Zoutendijk E, Barkun AN, Bardou M (2020) Recent advances in clinical practice: colorectal cancer chemoprevention in the average-risk population. Gut 69(12):2244-2255

Chassot F, Pozzebon Venturini T, Baldissera Piasentin F, Morais Santurio J, Estivalet Svidzinski TI, Hartz Alves S (2016) Antifungal activities of diphenyl diselenide and ebselen against echinocandin-susceptible and-resistant strains of Candida parapsilosis. New Microbiol 39:301-303

Chaudiere J, Courtin O, Leclaire J (1992) Glutathione oxidase activity of selenocystamine: a mechanistic study. Arch Biochem Biophys 296(1):328-336

Chen T, Wong Y-S (2009) Selenocystine induces caspase-independent apoptosis in MCF-7 human breast carcinoma cells with involvement of p53 phosphorylation and reactive oxygen species generation. Int J Biochem Cell Biol 41(3):666-676

Chen C, Yang K (2019) Ebselen bearing polar functionality: Identification of potent antibacterial agents against multidrug-resistant Gram-negative bacteria. Bioorg Chem 93:103286

Chen X, Chen H, Zhang H et al (2019) Characterization of synergistic antibacterial effect of silver nanoparticles and ebselen. Artif Cells Nanomed Biotechnol 47(1):3338-3349

Chen Z, Lai H, Hou L, Chen T (2020) Rational design and action mechanisms of chemically innovative organoselenium in cancer therapy. Chem Commun 56(2):179-196

Cheng B, Zhong JP, Wu FX et al (2019) Ebselen protects rat hearts against myocardial ischemia-reperfusion injury. Exper Ther Med 17(2):1412-1419

Choi Y-B, Lipton S (2000) Redox modulation of the NMDA receptor. Cell Mol Life Sci CMLS 57(11):1535-1541

Christensen MJ, Quiner TE, Nakken HL, Lephart ED, Eggett DL, Urie PM (2013) Combination effects of dietary soy and methylselenocysteine in a mouse model of prostate cancer. Prostate 73(9):986-995

Clark LC, Combs GF, Turnbull BW et al (1996) Effects of selenium supplementation for cancer prevention in patients with 
carcinoma of the skin: a randomized controlled trial. JAMA 276(24):1957-1963

Cone JE, Del Rio RM, Davis JN, Stadtman TC (1976) Chemical characterization of the selenoprotein component of clostridial glycine reductase: identification of selenocysteine as the organoselenium moiety. Proc Natl Acad Sci 73(8):2659-2663

Costa MD, Gai BM, Acker CI, Souza ACG, Brandão R, Nogueira CW (2012) Ebselen reduces hyperglycemia temporarily-induced by diazinon: a compound with insulin-mimetic properties. Chem Biol Interact 197(2-3):80-86

Costa MD, de Freitas ML, Dalmolin L et al (2013) Diphenyl diselenide prevents hepatic alterations induced by paraquat in rats. Environ Toxicol Pharmacol 36(3):750-758

Cotgreave I, Johansson U, Westergren G, Moldeus P, Brattsand R (1988) The anti-inflammatory activity of Ebselen but not thiols in experimental alveolitis and bronchiolitis. Agents Actions 24(3-4):313-319

Cotgreave IA, Duddy SK, Kass GE, Thompson D, Moldéus P (1989) Studies on the anti-inflammatory activity of Ebselen: Ebselen interferes with granulocyte oxidative burst by dual inhibition of NADPH oxidase and protein kinase C? Biochem Pharmacol 38(4):649-656

Cryan LM, Habeshian KA, Caldwell TP et al (2013) Identification of small molecules that inhibit the interaction of TEM8 with anthrax protective antigen using a FRET assay. J Biomol Screen 18(6):714-725

da Luz FT, Oliveira CS, da Costa M, Oliveira VA, Zeni G, Pereira ME (2015) Effectiveness of (PhSe) 2 in protect against the $\mathrm{HgCl}_{2}$ toxicity. J Trace Elem Med Biol 29:255-262

da Rocha JT, Pinton S, Mazzanti A et al (2011) Effects of diphenyl diselenide on lipid profile and hepatic oxidative stress parameters in ovariectomized female rats. J Pharm Pharmacol 63(5):663-669

da Rocha JT, Pinton S, Mazzanti A et al (2012b) Diphenyl diselenide ameliorates cognitive deficits induced by a model of menopause in rats. Behav Pharmacol 23(1):98-104

da Rocha JT, Trapani L, Segatto M et al (2013b) Molecular effects of diphenyl diselenide on cholesterol and glucose cell metabolism. Curr Med Chem 20(35):4426-4434

da Rosa EJ, da Silva MH, Carvalho NR et al (2012) Reduction of acute hepatic damage induced by acetaminophen after treatment with diphenyl diselenide in mice. Toxicol Pathol 40(4):605-613

da Silva MH, da Rosa EJF, de Carvalho NR et al (2012) Acute brain damage induced by acetaminophen in mice: effect of diphenyl diselenide on oxidative stress and mitochondrial dysfunction. Neurotox Res 21(3):334-344

Da Rocha JT, Sperança A, Nogueira CW, Zeni G (2009) Hypolipidaemic activity of orally administered diphenyl diselenide in Triton WR-1339-induced hyperlipidaemia in mice. J Pharm Pharmacol 61(12):1673-1679

Da Rocha JT, Gai BM, Pinton S, Sampaio TB, Nogueira CW, Zeni G (2012a) Effects of diphenyl diselenide on depressive-like behavior in ovariectomized mice submitted to subchronic stress: involvement of the serotonergic system. Psychopharmacology 222(4):709-719

Da Rocha JT, Pinton S, Gai BM, Nogueira CW (2013a) Diphenyl diselenide reduces mechanical and thermal nociceptive behavioral responses after unilateral intrastriatal administration of 6-hydroxydopamine in rats. Biol Trace Elem Res 154(3):372-378

Dalla Corte CL, Bastos LL, Dobrachinski F, Rocha JB, Soares FA (2012) The combination of organoselenium compounds and guanosine prevents glutamate-induced oxidative stress in different regions of rat brains. Brain Res 1430:101-111

Dalla Corte CL, Wagner C, Sudati JH et al (2013) Effects of diphenyl diselenide on methylmercury toxicity in rats. BioMed Res Int 983821:1-13
Dalla Corte CL, Ramos A, dos Santos CMM, Dressler VL, da Rocha JBT (2016) Selenium and mercury levels in rat liver slices cotreated with diphenyl diselenide and methylmercury. Biometals 29(3):543-550

de Freitas AS, Rocha JBT (2011) Diphenyl diselenide and analogs are substrates of cerebral rat thioredoxin reductase: a pathway for their neuroprotective effects. Neurosci Lett 503(1):1-5

de Andrade RB, Gemelli T, Guerra RB et al (2014) Acute exposure to the vinyl chalcogenide 3-methyl-1-phenyl-2-(phenylseleno) oct-2-en-1-one induces oxidative stress in different brain area of rats. Cell Biochem Funct 32(5):438-444

de Bem AF, de Lima PR, Perottoni J et al (2006) Changes in biochemical parameters in rabbits blood after oral exposure to diphenyl diselenide for long periods. Chem Biol Interact 162(1):1-10

de Bem AF, Farina M, de Lima PR et al (2008) Diphenyl diselenide, a simple glutathione peroxidase mimetic, inhibits human LDL oxidation in vitro. Atherosclerosis 201(1):92-100

de Bem AF, Fiuza B, Calcerrada P et al (2013) Protective effect of diphenyl diselenide against peroxynitrite-mediated endothelial cell death: a comparison with ebselen. Nitric Oxide 31:20-30

de Freitas AS, Funck VR, dos Santos RM et al (2009) Diphenyl diselenide, a simple organoselenium compound, decreases methylmercury-induced cerebral, hepatic and renal oxidative stress and mercury deposition in adult mice. Brain Res Bull 79(1):77-84

de Freitas ML, da Silva AR, Roman SS, Brandão R (2012) Effects of 4,4'-dichloro-diphenyl diselenide ( $\mathrm{ClPhSe}) 2$ on toxicity induced by mercuric chloride in mice: a comparative study with diphenyl diselenide (PhSe) 2. Environ Toxicol Pharmacol 34(3):985-994

de Menezes CC, Leitemperger J, Santi A et al (2012) The effects of diphenyl diselenide on oxidative stress biomarkers in Cyprinus carpio exposed to herbicide quinclorac (Facet $\left.{ }^{\circledR}\right)$. Ecotoxicol Environ Saf 81:91-97

de Munnik M, Lohans CT, Lang PA et al (2019) Targeting the Mycobacterium tuberculosis transpeptidase Ldt Mt2 with cysteine-reactive inhibitors including ebselen. Chem Commun 55(69):10214-10217

de Oliveira IM, Degrandi TH, Jorge PM et al (2014) Dicholesteroyl diselenide: cytotoxicity, genotoxicity and mutagenicity in the yeast Saccharomyces cerevisiae and in Chinese hamster lung fibroblasts. Mutat Res Genet Toxicol Environ Mutagen 763:1-11

de Souza D, Mariano DO, Nedel F et al (2015) New organochalcogen multitarget drug: synthesis and antioxidant and antitumoral activities of chalcogenozidovudine derivatives. J Med Chem 58(8):3329-3339

De Bem AF, de Lima PR, Colpo E et al (2009) Diphenyl diselenide decreases serum levels of total cholesterol and tissue oxidative stress in cholesterol-fed rabbits. Basic Clin Pharmacol Toxicol 105(1):17-23

De Oliveira HC, Monteiro MC, Rossi SA et al (2019) Identification of off-patent compounds that present antifungal activity against the emerging fungal pathogen Candida auris. Front Cell Infect Microbiol 9:83

De-Mello MA, Flodström M, Eizirik DL (1996) Ebselen and cytokineinduced nitric oxide synthase expression in insulin-producing cells. Biochem Pharmacol 52(11):1703-1709

Denardi LB, Mario DAN, de Loreto ES, Nogueira CW, Santurio JM, Alves SH (2013) Antifungal activities of diphenyl diselenide alone and in combination with fluconazole or amphotericin B against Candidaglabrata. Mycopathologia 176(1-2):165-169

Dhanarajan R, Abraham P, Isaac B (2006) Protective effect of ebselen, a selenoorganic drug, against gentamicin-induced renal damage in rats. Basic Clin Pharmacol Toxicol 99(3):267-272 
Dhanasekaran M, Uthayathas S, Karuppagounder SS et al (2006) Ebselen effects on MPTP-induced neurotoxicity. Brain Res 1118(1):251-254

Dhingra S, Bansal MP (2005) Hypercholesterolemia and apolipoprotein B expression: regulation by selenium status. Lipids Health Dis 4(1):28

Dhingra S, Bansal MP (2006) Hypercholesterolemia and LDL receptor mRNA expression: modulation by selenium supplementation. Biometals 19(5):493-501

Di Leo I, Messina F, Nascimento V et al (2019) Synthetic approaches to organoselenium derivatives with antimicrobial and anti-biofilm activity. Mini-Rev Org Chem 16(6):589-601

Dias GRM, Vieira FA, Dobrachinski F et al (2012) Diphenyl diselenide diet intake improves spatial learning and memory deficits in hypothyroid female rats. Int J Dev Neurosci 30(2):83-89

Dias GRM, de Almeida TM, Sudati JH et al (2014) Diphenyl diselenide supplemented diet reduces depressive-like behavior in hypothyroid female rats. Physiol Behav 124:116-122

Díaz M, González R, Plano D, Palop JA, Sanmartín C, Encío I (2018) A diphenyldiselenide derivative induces autophagy via JNK in HTB-54 lung cancer cells. J Cell Mol Med 22(1):289-301

Dimmeler S, Brüne B, Ullrich V (1991) Ebselen prevents inositol (1, $4,5)$-trisphosphate binding to its receptor. Biochem Pharmacol 42(5):1151-1153

Dinh QT, Cui Z, Huang J et al (2018) Selenium distribution in the Chinese environment and its relationship with human health: a review. Environ Int 112:294-309

Dobrachinski F, da Silva MH, Tassi CLC et al (2014) Neuroprotective effect of diphenyl diselenide in a experimental stroke model: maintenance of redox system in mitochondria of brain regions. Neurotox Res 26(4):317-330

Doleski PH, Leal DB, Machado VS et al (2017a) Diphenyl diselenide modulates nucleotidases, reducing inflammatory responses in the liver of Toxoplasma gondii-infected mice. Purinergic Signal 13(4):489-496

Doleski PH, Ten Caten MV, Passos DF et al (2017b) Toxoplasmosis treatment with diphenyl diselenide in infected mice modulates the activity of purinergic enzymes and reduces inflammation in spleen. Exp Parasitol 181:7-13

Domingues M, Casaril AM, Birmann PT et al (2018) Selanylimidazopyridine prevents lipopolysaccharide-induced depressive-like behavior in mice by targeting neurotrophins and inflammatory/ oxidative mediators. Front Neurosci 12:486

Domingues M, Casaril AM, Birmann PT et al (2019) Effects of a selanylimidazopyridine on the acute restraint stress-induced depressive-and anxiety-like behaviors and biological changes in mice. Behav Brain Res 366:96-107

Donato F, de Gomes MG, Goes ATR et al (2013) Involvement of the dopaminergic and serotonergic systems in the antidepressant-like effect caused by 4-phenyl-1-(phenylselanylmethyl)-1, 2, 3-triazole. Life Sci 93(9-11):393-400

Donato F, Pavin NF, Goes ATR et al (2015) Antinociceptive and antihyperalgesic effects of bis (4-methylbenzoyl) diselenide in mice: evidence for the mechanism of action. Pharm Biol 53(3):395-403

Dong J, Chen H (2018) Cardiotoxicity of anti-cancer therapeutics. Front Cardiovasc Med 5:9

Dong C, Wang J, Chen H et al (2020) Synergistic therapeutic efficacy of ebselen and silver ions against multidrug-resistant Acinetobacter baumannii-induced urinary tract infections. Metallomics 12(6):860-867

dos Santos LD, de Oliveira CV, Mascarenhas M et al (2012) Acute administration of the organochalcogen 3-methyl-1-phenyl-2(phenylseleno) oct-2-en-1-one induces biochemical and hematological disorders in male rats. Cell Biochem Funct 30(4):315-319

dos Santos MM, de Macedo GT, Prestes AS et al (2018) Hyperglycemia elicits anxiety-like behaviors in zebrafish: protective role of dietary diphenyl diselenide. Prog Neuropsychopharmacol Biol Psychiatry 85:128-135

dos Santos MM, de Macedo GT, Prestes AS et al (2020) Modulation of Redox and insulin signaling underlie the anti-hyperglycemic and antioxidant effects of diphenyl diselenide in zebrafish. Free Radic Biol Med 158:20-31

Drummond E, Wisniewski T (2017) Alzheimer's disease: experimental models and reality. Acta Neuropathol 133(2):155-175

Duarte LFB, Oliveira RL, Rodrigues KC et al (2017) Organoselenium compounds from purines: synthesis of 6-arylselanylpurines with antioxidant and anticholinesterase activities and memory improvement effect. Bioorg Med Chem 25(24):6718-6723

Duffield-Lillico AJ, Slate EH, Reid ME et al (2003) Selenium supplementation and secondary prevention of nonmelanoma skin cancer in a randomized trial. J Natl Cancer Inst 95(19):1477-1481

Dunn BK, Richmond ES, Minasian LM, Ryan AM, Ford LG (2010) A nutrient approach to prostate cancer prevention: the selenium and vitamin E cancer prevention trial (SELECT). Nutr Cancer 62(7):896-918

Duntas LH, Benvenga S (2015) Selenium: an element for life. Endocrine 48(3):756-775

Ecker A, Ledur PC, da Silva RS et al (2017) Chalcogenozidovudine derivatives with antitumor activity: comparative toxicities in cultured human mononuclear cells. Toxicol Sci 160(1):30-46

Ecker A, da Silva RS, dos Santos MM et al (2018) Safety profile of AZT derivatives: organoselenium moieties confer different cytotoxic responses in fresh human erythrocytes during in vitro exposures. J Trace Elem Med Biol 50:240-248

El-Bayoumy K (1985) Effects of organoselenium compounds on induction of mouse forestomach tumors by benzo (a) pyrene. Can Res 45(8):3631-3635

El-Bayoumy K, Sinha R (2004) Mechanisms of mammary cancer chemoprevention by organoselenium compounds. Mutat Res Fundam Mol Mech Mutagen 551(1-2):181-197

Eldabaje R, Le DL, Huang W, Yang L-X (2015) Radiation-associated cardiac injury. Anti-cancer Res 35(5):2487-2492

Elsherbini M, Hamama WS, Zoorob HH, Bhowmick D, Mugesh G, Wirth T (2014) Synthesis and antioxidant activities of novel chiral ebselen analogues. Heteroat Chem 25(5):320-325

Elsherbini M, Hamama WS, Zoorob HH (2016) Recent advances in the chemistry of selenium-containing heterocycles: five-membered ring systems. Coord Chem Rev 312:149-177

Evans SO, Jacobson GM, Goodman HJ, Bird S, Jameson MB (2020) Comparison of three oral selenium compounds in cancer patients: evaluation of differential pharmacodynamic effects in normal and malignant cells. J Trace Elem Med Biol 58:126446

Fachinetto R, Villarinho JG, Wagner C et al (2007) Diphenyl diselenide decreases the prevalence of vacuous chewing movements induced by fluphenazine in rats. Psychopharmacology 194(3):423-432

Falk M, Bernhoft A, Reinoso-Maset E et al (2020) Beneficial antioxidant status of piglets from sows fed selenomethionine compared with piglets from sows fed sodium selenite. J Trace Elem Med Biol 58:126439

Farina M, Barbosa N, Nogueira C et al (2002) Reaction of diphenyl diselenide with hydrogen peroxide and inhibition of delta-aminolevulinate dehydratase from rat liver and cucumber leaves. Braz J Med Biol Res 35(6):623-631

Farina M, Soares F, Zeni G, Souza D, Rocha J (2004) Additive prooxidative effects of methylmercury and ebselen in liver from suckling rat pups. Toxicol Lett 146(3):227-235

Favrot L, Grzegorzewicz AE, Lajiness DH et al (2013) Mechanism of inhibition of Mycobacterium tuberculosis antigen 85 by ebselen. Nat Commun 4(1):1-10

Felli Kubiça T, Bedin Denardi L, Silva de Loreto É et al (2019) In vitro activity of diphenyl diselenide and ebselen alone and in 
combination with antifungal agents against Trichosporon asahii. Mycoses 62(5):428-433

Feng Q, Li X, Sun W et al (2020) Discovery of ebselen as an inhibitor of 6PGD for suppressing tumor growth. Cancer Manag Res $12: 6921$

Ferreira LM, Azambuja JH, da Silveira EF et al (2019a) Antitumor action of diphenyl diselenide nanocapsules: In vitro assessments and preclinical evidence in an animal model of glioblastoma multiforme. J Trace Elem Med Biol 55:180-189

Ferreira LM, da Rosa LVC, Müller TE et al (2019b) Zebrafish exposure to diphenyl diselenide-loaded polymeric nanocapsules caused no behavioral impairments and brain oxidative stress. J Trace Elem Med Biol 53:62-68

Ferreira LM, Sari MHM, Azambuja JH et al (2020) Xanthan gumbased hydrogel containing nanocapsules for cutaneous diphenyl diselenide delivery in melanoma therapy. Investig New Drugs 38(3):662-674

Fiala ES, Joseph C, Sohn OS, El-Bayoumy K, Reddy BS (1991) Mechanism of benzylselenocyanate inhibition of azoxymethane-induced colon carcinogenesis in F344 rats. Can Res 51(11):2826-2830

Figueira FH, Leal CQ, de Moraes RE et al (2015) Effects of diphenyl diselenide on behavioral and biochemical changes induced by amphetamine in mice. J Neural Transm 122(2):201-209

Filho CB, Del Fabbro L, Boeira SP et al (2013) Hepatoprotective effect of bis (4-methylbenzoyl) diselenide against $\mathrm{CCl} 4$-induced oxidative damage in mice. Cell Biochem Funct 31(2):152-158

Fink LH, Anastasio NC, Fox RG, Rice KC, Moeller FG, Cunningham KA (2015) Individual differences in impulsive action reflect variation in the cortical serotonin 5-HT 2A receptor system. Neuropsychopharmacology 40(8): 1957-1968

Fischer H, Terlinden R, Lohr JP, Romer A (1988) A novel biologically active selenoorganic compound. VIII. Biotransformation of ebselen. Xenobiotica 18(12):1347-1359

Flohe L, Gunzler W, Schock H (1973) Glutathione peroxidase: a selenoenzyme. FEBS Lett 32(1):132-134

Fonseca SF, Lima DB, Alves D et al (2015) Synthesis, characterization and antioxidant activity of organoselenium and organotellurium compound derivatives of chrysin. New J Chem 39(4):3043-3050

Forstrom JW, Zakowski JJ, Tappel AL (1978) Identification of the catalytic site of rat liver glutathione peroxidase as selenocysteine. Biochemistry 17(13):2639-2644

Freeman EB (1922) A preliminary report on the treatment of carcinoma of the Esophagus with colloidal selenium. Boston Med Surg J 187(21):727-732

Fulco BCW, Jung JTK, Chagas PM, Rosa SG, Prado VC, Nogueira CW (2020) Diphenyl diselenide is as effective as Ebselen in a juvenile rat model of cisplatin-induced nephrotoxicity. J Trace Elem Med Biol 60:126482

Gai BM, Stein AL, Roehrs JA, Bilheri FN, Nogueira CW, Zeni G (2012) Synthesis and antidepressant-like activity of selenophenes obtained via iron (III)-PhSeSePh-mediated cyclization of Z-selenoenynes. Org Biomol Chem 10(4):798-807

Gai BM, Bortolatto CF, Brüning CA et al (2014a) Depression-related behavior and mechanical allodynia are blocked by 3-(4-fluorophenylselenyl)-2, 5-diphenylselenophene in a mouse model of neuropathic pain induced by partial sciatic nerve ligation. Neuropharmacology 79:580-589

Gai BM, Bortolatto CF, Heck SO et al (2014b) An organoselenium compound improves behavioral, endocrinal and neurochemical changes induced by corticosterone in mice. Psychopharmacology 231(10):2119-2130

Gai BM, Sanna MD, Stein AL, Zeni G, Galeotti N, Nogueira CW (2014c) ERK1/2 phosphorylation is involved in the antidepressant-like action of 2, 5-diphenyl-3-(4-fluorophenylseleno)-selenophene in mice. Eur J Pharmacol 736:44-54
Galant LS, Braga MM, de Souza D et al (2017) Induction of reactive oxygen species by diphenyl diselenide is preceded by changes in cell morphology and permeability in Saccharomyces cerevisiae. Free Radic Res 51(7-8):657-668

Galant LS, Rafique J, Braga AL et al (2020) The thiol-modifier effects of organoselenium compounds and their cytoprotective actions in neuronal cells. Neurochem Res 46:120-130

Galet V, Bernier J-L, Henichart J-P et al (1994) Benzoselenazolinone derivatives designed to be glutathione peroxidase mimetics feature inhibition of cyclooxygenase/5-lipoxygenase pathways and anti-inflammatory activity. J Med Chem 37(18):2903-2911

Gandin V, Khalkar P, Braude J, Fernandes AP (2018) Organic selenium compounds as potential chemotherapeutic agents for improved cancer treatment. Free Radic Biol Med 127:80-97

Ganther HE (1968) Selenotrisulfides. Formation by the reaction of thiols with selenious acid. Biochemistry 7(8):2898-2905

Gao J-X, Issekutz AC (1993) The effect of ebselen on polymorphonuclear leukocyte and lymphocyte migration to inflammatory reactions in rats. Immunopharmacology 25(3):239-251

García Sar D, Montes-Bayón M, Blanco González E, Sierra Zapico LM, Sanz-Medel A (2011) Reduction of cisplatin-induced nephrotoxicity in vivo by selenomethionine: the effect on cisplatin-DNA adducts. Chem Res Toxicol 24(6):896-904

Garnica P, Encío I, Plano D, Palop JA, Sanmartín C (2018) Combined acylselenourea-diselenide structures: new potent and selective antitumoral agents as autophagy activators. ACS Med Chem Lett 9(4):306-311

Gay BM, Prigol M, Stein AL, Nogueira CW (2010) Antidepressant-like pharmacological profile of 3-(4-fluorophenylselenyl)-2, 5-diphenylselenophene: Involvement of serotonergic system. Neuropharmacology 59(3):172-179

Gealekman O, Brodsky SV, Zhang F et al (2004) Endothelial dysfunction as a modifier of angiogenic response in Zucker diabetic fat rat: amelioration with Ebselen. Kidney Int 66(6):2337-2347

Gennadyevna VE (2020) Protein-protein interactions of ER-resident selenoproteins with their physiological partners. Biochimie 171-172:197-204

Gerzson MFB, Victoria FN, Radatz CS et al (2012) In vitro antioxidant activity and in vivo antidepressant-like effect of $\alpha$-(phenylselanyl) acetophenone in mice. Pharmacol Biochem Behav 102(1):21-29

Ghisleni G, Porciuncula LO, Cimarosti H, Rocha JBT, Salbego CG, Souza DO (2003) Diphenyl diselenide protects rat hippocampal slices submitted to oxygen-glucose deprivation and diminishes inducible nitric oxide synthase immunocontent. Brain Res 986(1-2):196-199

Ghisleni G, Kazlauckas V, Both FL et al (2008a) Diphenyl diselenide exerts anxiolytic-like effect in Wistar rats: putative roles of GABAA and 5HT receptors. Prog Neuropsychopharmacol Biol Psychiatry 32(6): 1508-1515

Ghisleni G, Porciúncula LO, Mioranzza S, Boeck CR, Rocha JB, Souza DO (2008b) Selenium compounds counteract the stimulation of ecto-nucleotidase activities in rat cultured cerebellar granule cells: putative correlation with neuroprotective effects. Brain Res 1221:134-140

Ghosh S (2019) Cisplatin: the first metal based anti-cancer drug. Bioorg Chem 88:102925

Ghosh P, Roy SS, Chakraborty P, Ghosh S, Bhattacharya S (2013) Effects of organoselenium compound 2-(5-selenocyanatopentyl)-benzo [de] isoquinoline 1,3-dione on cisplatin induced nephrotoxicity and genotoxicity: an investigation of the influence of the compound on oxidative stress and antioxidant enzyme system. Biometals 26(1):61-73

Ghosh P, Singha Roy S, Basu A, Bhattacharjee A, Bhattacharya S (2015) Sensitization of cisplatin therapy by a naphthalimide based organoselenium compound through modulation of 
antioxidant enzymes and p53 mediated apoptosis. Free Radic Res 49(4):453-471

Giurg M, Gołąb A, Suchodolski J et al (2017) Reaction of bis [(2-chlorocarbonyl) phenyl] diselenide with phenols, aminophenols, and other amines towards diphenyl diselenides with antimicrobial and antiviral properties. Molecules 22(6):974

Glaser V, Moritz B, Schmitz A et al (2013) Protective effects of diphenyl diselenide in a mouse model of brain toxicity. Chem Biol Interact 206(1):18-26

Glaser V, de Paula MR, Vieira AJH et al (2014) Diphenyl diselenide administration enhances cortical mitochondrial number and activity by increasing hemeoxygenase type 1 content in a methylmercury-induced neurotoxicity mouse model. Mol Cell Biochem 390(1-2):1-8

Godoi GL, de Oliveira PL, Schulz JF et al (2013) Selenium compounds prevent amyloid $\beta$-peptide neurotoxicity in rat primary hippocampal neurons. Neurochem Res 38(11):2359-2363

Goins CM, Dajnowicz S, Thanna S, Sucheck SJ, Parks JM, Ronning DR (2017) Exploring covalent allosteric inhibition of antigen 85C from Mycobacterium tuberculosis by ebselen derivatives. ACS Infect Dis 3(5):378-387

Gopalakrishna R, Gundimeda U, Zhou S, Bui H, Holmgren A (2018) Redox regulation of protein kinase $\mathrm{C}$ by selenometabolites and selenoprotein thioredoxin reductase limits cancer prevention by selenium. Free Radic Biol Med 127:55-61

Graham DY, Chan FK (2008) NSAIDs, risks, and gastroprotective strategies: current status and future. Gastroenterology 134(4):1240-1246

Guillin OM, Vindry C, Ohlmann T, Chavatte L (2019) Selenium, selenoproteins and viral infection. Nutrients 11(9):2101

Gunes S, Sahinturk V, Karasati P, Sahin IK, Ayhanci A (2017) Cardioprotective effect of selenium against cyclophosphamide-induced cardiotoxicity in rats. Biol Trace Elem Res 177(1):107-114

Gustafsson TN, Osman H, Werngren J, Hoffner S, Engman L, Holmgren A (2016) Ebselen and analogs as inhibitors of Bacillus anthracis thioredoxin reductase and bactericidal antibacterials targeting Bacillus species, Staphylococcus aureus and Mycobacterium tuberculosis. Biochim Biophys Acta (BBA) Gen Subj 1860(6):1265-1271

Halliwell B (2006) Phagocyte-derived reactive species: salvation or suicide? Trends Biochem Sci 31(9):509-515

Halliwell B (2012) Free radicals and antioxidants: updating a personal view. Nutr Rev 70(5):257-265

Halliwell B (2013) The antioxidant paradox: less paradoxical now? Br J Clin Pharmacol 75(3):637-644

Han HW, Yang EJ, Lee S-M (2019) Sodium selenite alleviates breast cancer-related lymphedema independent of antioxidant defense system. Nutrients 11(5):1021

Hanavan PD, Borges CR, Katchman BA et al (2015) Ebselen inhibits QSOX1 enzymatic activity and suppresses invasion of pancreatic and renal cancer cell lines. Oncotarget 6(21):18418

Handa E, Puspitasari IM, Abdulah R et al (2020) Recent advances in clinical studies of selenium supplementation in radiotherapy. $\mathbf{J}$ Trace Elem Med Biol 62:126653

Haritha C, Sharun K, Jose B (2020) Ebselen, a new candidate therapeutic against SARS-CoV-2. Int J Surg (Lond Engl) 84:53

Hassan W, Rocha JBT (2012) Interaction profile of diphenyl diselenide with pharmacologically significant thiols. Molecules 17(10): 12287-12296

Hassan W, Silva CEB, da Rocha JBT, Landeira-Fernandez J (2015) Modulatory effect of diphenyl diselenide in carioca high-and low-conditioned freezing rats. Eur J Pharmacol 761:341-344

Hassan W, Oliveira SC, Noreen H, Kamdem PJ, Nogueira WC, Rocha BTJ (2016) Organoselenium compounds as potential neuroprotective therapeutic agents. Curr Org Chem 20(2):218-231
Hatfield DL, Tsuji PA, Carlson BA, Gladyshev VN (2014) Selenium and selenocysteine: roles in cancer, health, and development. Trends Biochem Sci 39(3):112-120

Heck SO, Zborowski VA, Quines CB, Nogueira CW (2019) 4,4'-Dichlorodiphenyl diselenide reverses a depressive-like phenotype, modulates prefrontal cortical oxidative stress and dysregulated glutamatergic neurotransmission induced by subchronic dexamethasone exposure to mice. J Psychiatr Res 116:61-68

Heller RA, Sun Q, Hackler J et al (2020) Prediction of survival odds in COVID-19 by zinc, age and selenoprotein $\mathrm{P}$ as composite biomarker. Redox Biol 38:101764

Hodage AS, Phadnis PP, Wadawale A, Priyadarsini K, Jain VK (2012) Synthesis, Characterization, and X-ray structures of 2-(3, 5-dimethylpyrazol-1-Y1) phenyl-based organoselenium compounds and their glutathione peroxidase ( $\mathrm{Gp} \mathrm{x}$ ) like activity. Phosphorus Sulfur Silicon Relat Elem 187(12):1419-1433

Hort MA, Straliotto MR, Netto PM, da Rocha JBT, de Bem AF, Ribeiro-do-Valle RM (2011) Diphenyl diselenide effectively reduces atherosclerotic lesions in LDLr-/- mice by attenuation of oxidative stress and inflammation. J Cardiovasc Pharmacol 58(1):91-101

Howard MT, Copeland PR (2019) New directions for understanding the codon redefinition required for selenocysteine incorporation. Biol Trace Elem Res 192(1):18-25

Huang Z, Rose AH, Hoffmann PR (2012) The role of selenium in inflammation and immunity: from molecular mechanisms to therapeutic opportunities. Antioxid Redox Signal 16(7):705-743

Ibrahim M, Prigol M, Hassan W, Nogueira CW, Rocha JB (2010) Protective effect of binaphthyl diselenide, a synthetic organoselenium compound, on 2-nitropropane-induced hepatotoxicity in rats. Cell Biochem Funct Cell Biochem Modul Act Agents Dis 28(4):258-265

Ibrahim M, Luchese C, Pinton S et al (2011) Involvement of catalase in the protective effect of binaphthyl diselenide against renal damage induced by glycerol. Exp Toxicol Pathol 63(4):331-335

Ibrahim M, Hassan W, Deobald AM, Braga AL, Rocha JB (2012a) An Organoselenium drug with antioxidant activity and free radical scavenging capacity in vitro. Biol Trace Elem Res 149(3):399-404

Ibrahim M, Hassan W, Meinerz DF, de Oliveira LG, Nogueira CW, Rocha JB (2012b) Ethanol-induced oxidative stress: the role of binaphthyl diselenide as a potent antioxidant. Biol Trace Elem Res 147(1-3):309-314

Ibrahim M, Mussulini BHM, Moro L et al (2014b) Anxiolytic effects of diphenyl diselenide on adult zebrafish in a novelty paradigm. Prog Neuropsychopharmacol Biol Psychiatry 54:187-194

Ibrahim M, Hassan W, Anwar J et al (2014a) 1-(2-(2-(2-(1-Aminoethyl) phenyl) diselanyl) phenyl) ethanamine: an amino organoselenium compound with interesting antioxidant profile. Toxicol In Vitro 28(4):524-530

Ibrahim M, Muhammad N, Naeem M, Deobald AM, Kamdem JP, Rocha JBT (2015) In vitro evaluation of glutathione peroxidase (GPx)-like activity and antioxidant properties of an organoselenium compound. Toxicol In Vitro 29(5):947-952

Ibrahim M, Ibrahim M, Muhammad N, Shah MIA, de Oliveira LG, Rocha JB (2018) Pharmacological mechanisms underlying gastroprotective activities of binapthyl diselenide in Wistar rats. Inflammopharmacology 26(4):1117-1123

Ibrahim M, Muhammad N, Ibrahim M et al (2019) Acute oral toxicity and antioxidant studies of an amine-based diselenide. BMC Complement Altern Med 19(1):80

Ichikawa S, Omura K, Katayama T et al (1987) Inhibition of superoxide anion production in guinea pig polymorphonuclear leukocytes by A seleno-or-ganic compound. Ebselen J Pharm Dyn 10(10):595-597 
Ineu R, Pereira M, Aschner M, Nogueira C, Zeni G, Rocha J (2008) Diphenyl diselenide reverses gastric lesions in rats: involvement of oxidative stress. Food Chem Toxicol 46(9):3023-3029

Ip C (1998) Lessons from basic research in selenium and cancer prevention. J Nutr 128(11):1845-1854

Ip C, Ganther H (1992) Comparison of selenium and sulfur analogs in cancer prevention. Carcinogenesis 13(7):1167-1170

Ip C, Thompson HJ, Zhu Z, Ganther HE (2000) In vitro and in vivo studies of methylseleninic acid: evidence that a monomethylated selenium metabolite is critical for cancer chemoprevention. Can Res 60(11):2882-2886

Ismail HTH (2019) Hematobiochemical disturbances and oxidative stress after subacute manganese chloride exposure and potential protective effects of Ebselen in rats. Biol Trace Elem Res 187(2):452-463

Jablonska E, Vinceti M (2015) Selenium and human health: witnessing a Copernican revolution? J Environ Sci Health Part C 33(3):328-368

Jardim NS, Sartori G, Sari MH, Müller SG, Nogueira CW (2017) Bisphenol A impairs the memory function and glutamatergic homeostasis in a sex-dependent manner in mice: beneficial effects of diphenyl diselenide. Toxicol Appl Pharmacol 329:75-84

Jaromin A, Zarnowski R, Piętka-Ottlik M, Andes DR, Gubernator J (2018) Topical delivery of ebselen encapsulated in biopolymeric nanocapsules: drug repurposing enhanced antifungal activity. Nanomedicine 13(10):1139-1155

Jedrychowski MP, Lu GZ, Szpyt J et al (2020) Facultative protein selenation regulates redox sensitivity, adipose tissue thermogenesis, and obesity. Proc Natl Acad Sci 117(20):10789-10796

Jesse CR, Savegnago L, Nogueira CW (2007) Role of nitric oxide/ cyclic GMP/K+ channel pathways in the antinociceptive effect caused by 2, 3-bis (mesitylseleno) propenol. Life Sci 81(25-26):1694-1702

Jesse CR, Savegnago L, Nogueira CW (2008) Spinal mechanisms of antinociceptive effect caused by oral administration of bis-selenide in mice. Brain Res 1231:25-33

Jesse CR, Rocha JB, Nogueira CW, Savegnago L (2009) Further analysis of the antinociceptive action caused by $p$-methoxyl-diphenyl diselenide in mice. Pharmacol Biochem Behav 91(4):573-580

Jesse CR, Wilhelm EA, Bortolatto CF, Nogueira CW (2011) Role of different types of potassium channels and peroxisome proliferator-activated receptors $\gamma$ in the antidepressant-like activity of bis selenide in the mouse tail suspension test. Neurosci Lett 490(3):205-208

Jia Z-Q, Li S-Q, Qiao W-Q et al (2018) Ebselen protects mitochondrial function and oxidative stress while inhibiting the mitochondrial apoptosis pathway after acute spinal cord injury. Neurosci Lett 678:110-117

Jiang C, Jiang W, Ip C, Ganther H, Lu J (1999) Selenium-induced inhibition of angiogenesis in mammary cancer at chemopreventive levels of intake. Mol Carcinog 26(4):213-225

Jiang J-H, Zhou H, Li H-J et al (2015) Comprehensive modeling of the antioxidant mechanism of ebselen. J Theor Comput Chem 14(07): 1550053

Jiang Q, Pan Y, Cheng Y, Li H, Li H (2016) Protection of rat liver against hepatic ischemia-reperfusion injury by a novel selenocysteine-containing 7-mer peptide. Mol Med Rep 14(3):2007-2015

Jin Z, Du X, Xu Y et al (2020) Structure of M pro from SARS-CoV-2 and discovery of its inhibitors. Nature 582:289-293

Jung HJ, Kim HL, Kim YJ, Weon J-I, Seo YR (2013) A novel chemopreventive mechanism of selenomethionine: enhancement of APE1 enzyme activity via a Gadd45a, PCNA and APE1 protein complex that regulates p53-mediated base excision repair. Oncol Rep 30(4):1581-1586
Junior OdRA, Antônio E, Mainardes RM, Khalil NM (2017) Preparation, physicochemical characterization and antioxidant activity of diphenyl diselenide-loaded poly (lactic acid) nanoparticles. J Trace Elem Med Biol 39:176-185

Jupp L, Giles G (2012) Ebselen as a redox based therapeutic for triple negative breast cancer. Free Radic Biol Med 53:S98-S99

Kaczor-Keller KB, Pawlik A, Scianowski J et al (2020) In vitro antiprostate cancer activity of two ebselen analogues. Pharmaceuticals 13(3):47

Kade I, Borges V, Savegnago L et al (2009) Effect of oral administration of diphenyl diselenide on antioxidant status, and activity of delta aminolevulinic acid dehydratase and isoforms of lactate dehydrogenase, in streptozotocin-induced diabetic rats. Cell Biol Toxicol 25(4):415-424

Kalayci M, Coskun O, Cagavi F et al (2005) Neuroprotective effects of ebselen on experimental spinal cord injury in rats. Neurochem Res 30(3):403-410

Kamwesiga J, Mutabazi V, Kayumba J et al (2015) Effect of selenium supplementation on CD4+ T-cell recovery, viral suppression and morbidity of HIV-infected patients in Rwanda: a randomized controlled trial. AIDS (Lond Engl) 29(9):1045

Kassam S, Goenaga-Infante H, Maharaj L, Hiley CT, Juliger S, Joel SP (2011) Methylseleninic acid inhibits HDAC activity in diffuse large B-cell lymphoma cell lines. Cancer Chemother Pharmacol 68(3):815

Kil J, Lobarinas E, Spankovich C et al (2017) Safety and efficacy of ebselen for the prevention of noise-induced hearing loss: a randomised, double-blind, placebo-controlled, phase 2 trial. Lancet 390(10098):969-979

Kizilgun M, Poyrazoglu Y, Oztas Y et al (2011) Beneficial effects of $N$-acetylcysteine and ebselen on renal ischemia/reperfusion injury. Ren Fail 33(5):512-517

Klann IP, Martini F, Rosa SG, Nogueira CW (2020) Ebselen reversed peripheral oxidative stress induced by a mouse model of sporadic Alzheimer's disease. Mol Biol Rep 47(3):2205-2215

Klein EA, Thompson IM, Tangen CM et al (2011) Vitamin E and the risk of prostate cancer: the Selenium and Vitamin E Cancer Prevention Trial (SELECT). JAMA 306(14):1549-1556

Klimaczewski CV, Nogara PA, Barbosa NV, da Rocha JBT (2018) Interaction of metals from group $10(\mathrm{Ni}, \mathrm{Pd}$, and $\mathrm{Pt})$ and 11 $(\mathrm{Cu}, \mathrm{Ag}$, and $\mathrm{Au})$ with human blood $\delta$-ALA-D: in vitro and in silico studies. Environ Sci Pollut Res 25(30):30557-30566

Knox SJ, Jayachandran P, Keeling CA et al (2019) Results from a phase 1 study of sodium selenite in combination with palliative radiation therapy in patients with metastatic cancer. Transl Oncol 12(11):1525-1531

Koeberle A, Werz O (2014) Multi-target approach for natural products in inflammation. Drug Discov Today 19(12):1871-1882

Kohler LN, Foote J, Kelley CP et al (2018) Selenium and type 2 diabetes: systematic review. Nutrients 10(12):1924

Kolivand S, Amini P, Saffar H et al (2019) Selenium-L-methionine modulates radiation injury and Duox 1 and Duox 2 upregulation in rat's heart tissues. J Cardiovasc Thorac Res 11(2):121

Kono H, Arteel GE, Rusyn I, Sies H, Thurman RG (2001) Ebselen prevents early alcohol-induced liver injury in rats. Free Radic Biol Med 30(4):403-411

Korbut E, Ptak-Belowska A, Brzozowski T (2018) Inhibitory effect of selenomethionine on carcinogenesis in the model of human colorectal cancer in vitro and its link to the $\mathrm{Wnt} / \beta$-catenin pathway. Acta Biochim Polon 65(3):359-366

Kosten L, Chowdhury GM, Mingote S et al (2019) Glutaminase activity in GLS1 Het mouse brain compared to putative pharmacological inhibition by ebselen using ex vivo MRS. Neurochem Int 129:104508

Koyanagi T, Nakamuta M, Enjoji M et al (2001) The selenoorganic compound ebselen suppresses liver injury induced by 
Propionibacterium acnes and lipopolysaccharide in rats. Int $\mathbf{J}$ Mol Med 7(3):321-327

Krasowska D, Iraci N, Santi C et al (2019) Diselenides and benzisoselenazolones as antiproliferative agents and glutathione$S$-transferase inhibitors. Molecules 24(16):2914

Kuhl P, Borbe HO, Fischer H, Römer A, Safayhi H (1986) Ebselen reduces the formation of LTB4 in human and porcine leukocytes by isomerisation to its $5 \mathrm{~S}, 12 \mathrm{R}-6$-trans-isomer. Prostaglandins 31(6): 1029-1048

Kumar BS, Kunwar A, Singh BG, Ahmad A, Priyadarsini KI (2011) Anti-hemolytic and peroxyl radical scavenging activity of organoselenium compounds: an in vitro study. Biol Trace Elem Res 140(2):127-138

Kunwar A, Patil A, Kumar S et al (2018) Toxicological safety evaluation of 3, 3'-diselenodipropionic acid (DSePA), a pharmacologically important derivative of selenocystine. Regul Toxicol Pharmacol 99:159-167

Kunwar A, Priyadarsini KI, Jain VK (2020) 3, 3'-diselenodipropionic acid (DSePA): a redox active multifunctional molecule of biological relevance. Biochim Biophys Acta (BBA) Gen Subj 1865(1): 129768

Kurebayashi Y, Tabuchi Y, Akasaki M (1989) Gastric cytoprotection by ebselen against the injury induced by necrotizing agents in rats. Arzneimittelforschung 39(2):250

Kuria A, Tian H, Li M et al (2020) Selenium status in the body and cardiovascular disease: a systematic review and meta-analysis. Crit Rev Food Sci Nutr 10(1080/10408398):1803200

Labunskyy VM, Lee BC, Handy DE, Loscalzo J, Hatfield DL, Gladyshev VN (2011) Both maximal expression of selenoproteins and selenoprotein deficiency can promote development of type 2 diabetes-like phenotype in mice. Antioxid Redox Signal 14(12):2327-2336

Labunskyy VM, Hatfield DL, Gladyshev VN (2014) Selenoproteins: molecular pathways and physiological roles. Physiol Rev 94(3):739-777

Lafin JT, Sarsour EH, Kalen AL, Wagner BA, Buettner GR, Goswami PC (2019) Methylseleninic acid induces lipid peroxidation and radiation sensitivity in head and neck cancer cells. Int J Mol Sci 20(1):225

Leal M, Rocha J, Corte C et al (2018) Distribution of selenium in sheep treated with dipheny diselenide. Arq Bras Med Vet Zootec 70(4):1017-1022

Leão MB, da Rosa PC, Wagner C, Lugokenski TH, Dalla Corte CL (2018) Methylmercury and diphenyl diselenide interactions in Drosophila melanogaster: effects on development, behavior, and $\mathrm{Hg}$ levels. Environ Sci Pollut Res 25(22):21568-21576

Lee O, Moon J, Chung Y (2003) The relationship between serum selenium levels and lipid profiles in adult women. J Nutr Sci Vitaminol 49(6):397-404

Lee J-I, Nian H, Cooper AJ et al (2009) $\alpha$-Keto acid metabolites of naturally occurring organoselenium compounds as inhibitors of histone deacetylase in human prostate cancer cells. Cancer Prev Res 2(7):683-693

Lee J, Hong S, Sun J-H et al (2019) Toxicity of dietary selenomethionine in juvenile steelhead trout, Oncorhynchus mykiss: tissue burden, growth performance, body composition, hematological parameters, and liver histopathology. Chemosphere 226:755-765

Leite MR, Sari MHM, de Freitas ML et al (2014) Caffeine and diphenyl diselenide improve long-term memory impaired in middle-aged rats. Exp Gerontol 53:67-73

Leite MR, Cechella JL, Mantovani AC, Duarte MM, Nogueira CW, Zeni G (2015) Swimming exercise and diphenyl diselenide-supplemented diet affect the serum levels of pro-and anti-inflammatory cytokines differently depending on the age of rats. Cytokine 71(1):119-123
Lettieri-Barbato D (2019) Redox control of non-shivering thermogenesis. Mol Metabol 25:11-19

Leurs R, Timmerman H, Bast A (1989) Inhibition of superoxide anion radical production by ebselen (PZ51) and its sulfur analogue (PZ25) in guinea pig alveolar macrophages. Biochem Int 18(2):295-299

Levine VE (1915) Biochemical studies of selenium. Ann N Y Acad Sci 26(1):385-394

Levine VE (1925) The effect of selenium compounds upon growth and germination in plants. Am J Bot 12(2):82-90

Leyck S, Parnham M (1990) Acute antiinflammatory and gastric effects of the seleno-organic compound ebselen. Agents Actions 30(3-4):426-431

Li G-x, Lee H-J, Wang $Z$ et al (2008) Superior in vivo inhibitory efficacy of methylseleninic acid against human prostate cancer over selenomethionine or selenite. Carcinogenesis 29(5):1005-1012

Lippman SM, Klein EA, Goodman PJ et al (2009) Effect of selenium and vitamin $\mathrm{E}$ on risk of prostate cancer and other cancers: the Selenium and Vitamin E Cancer Prevention Trial (SELECT). JAMA 301(1):39-51

Liu Y, Liu X, Guo Y et al (2015b) Methylselenocysteine preventing castration-resistant progression of prostate cancer. Prostate 75(9):1001-1008

Liu K, Zhu C, Min J, Peng S, Xu G, Sun J (2015a) Stereodivergent synthesis of $N$-heterocycles by catalyst-controlled, activity-directed tandem annulation of diazo compounds with amino alkynes. Angew Chem Int Ed 54(44):12962-12967

Liu J, Wang S, Zhang Q, Li X, Xu S (2020a) Selenomethionine alleviates LPS-induced chicken myocardial inflammation by regulating the miR-128-3p-p38 MAPK axis and oxidative stress. Metallomics 12(1):54-64

Liu Z, Zhang F, Cui L et al (2020b) Quantitative proteomic analysis reveals that the Rap1/MAPK/ERK pathway is inhibited through selenomethionine strengthening antioxidant activity. Biometals 33(1):45-64

Loef M, Schrauzer GN, Walach H (2011) Selenium and Alzheimer's disease: a systematic review. J Alzheimer's Dis 26(1):81-104

Loomba R, Filippini T, Chawla R et al (2020) Exposure to a high selenium environment in Punjab, India: effects on blood chemistry. Sci Total Environ 716:135347

Loreto ÉS, Alves SH, Santurio JM, Nogueira CW, Zeni G (2012) Diphenyl diselenide in vitro and in vivo activity against the oomycete Pythium insidiosum. Vet Microbiol 156(1-2):222-226

Lu J, Jiang C, Kaeck M et al (1995) Dissociation of the genotoxic and growth inhibitory effects of selenium. Biochem Pharmacol 50(2):213-219

Lubos E, Sinning CR, Schnabel RB et al (2010) Serum selenium and prognosis in cardiovascular disease: results from the AtheroGene study. Atherosclerosis 209(1):271-277

Luchese C, Brandão R, Acker CI, Nogueira CW (2012a) 2, 2'-Dipyridyl diselenide is a better antioxidant than other disubstituted diaryl diselenides. Mol Cell Biochem 367(1-2):153-163

Luchese C, Prigol M, Duarte MM, Nogueira CW (2012b) Diphenyl diselenide reduces inflammation in the mouse model of pleurisy induced by carrageenan: reduction of pro-inflammatory markers and reactive species levels. Inflamm Res 61(10):1117-1124

Lugokenski TH, Müller LG, Taube PS, Rocha JB, Pereira ME (2011) Inhibitory effect of ebselen on lactate dehydrogenase activity from mammals: a comparative study with diphenyl diselenide and diphenyl ditelluride. Drug Chem Toxicol 34(1):66-76

Luo Z, Sheng J, Sun Y et al (2013) Synthesis and evaluation of multitarget-directed ligands against Alzheimer's disease based on the fusion of donepezil and ebselen. J Med Chem 56(22):9089-9099

Luo Z, Liang L, Sheng J et al (2014) Synthesis and biological evaluation of a new series of ebselen derivatives as glutathione 
peroxidase (GPx) mimics and cholinesterase inhibitors against Alzheimer's disease. Bioorg Med Chem 22(4):1355-1361

Lynch E, Kil J Development of ebselen, a glutathione peroxidase mimic, for the prevention and treatment of noise-induced hearing loss. In: Seminars in hearing, 2009. vol 30. (C) Thieme Medical Publishers, p 047-055

Lynch ED, Gu R, Pierce C, Kil J (2005) Reduction of acute cisplatin ototoxicity and nephrotoxicity in rats by oral administration of allopurinol and ebselen. Hear Res 201(1-2):81-89

Ma C, Hu Y, Townsend JA et al (2020) Ebselen, disulfiram, carmofur, PX-12, tideglusib, and shikonin are nonspecific promiscuous SARS-CoV-2 main protease inhibitors. ACS Pharmacol Transl Sci 3:1265-1277

Macegoniuk K, Grela E, Palus J et al (2016) 1, 2-Benzisoselenazol-3 $(2 \mathrm{H})$-one derivatives as a new class of bacterial urease inhibitors. J Med Chem 59(17):8125-8133

Machado MS, Rosa RM, Dantas AS et al (2006) An organic selenium compound attenuates apomorphine-induced stereotypy in mice. Neurosci Lett 410(3):198-202

Machado VS, Bottari NB, Baldissera MD et al (2016) Toxoplasma gondii: effects of diphenyl diselenide in experimental toxoplasmosis on biomarkers of cardiac function. Exp Parasitol 167:25-31

Madabeni A, Dalla Tiezza M, Omage FB et al (2020) Chalcogenmercury bond formation and disruption in model Rabenstein's reactions: a computational analysis. J Comput Chem 41(23):2045-2054

Magi S, Piccirillo S, Amoroso S (2019) The dual face of glutamate: from a neurotoxin to a potential survival factor-metabolic implications in health and disease. Cell Mol Life Sci 76(8):1473-1488

Mahadevan J, Parazzoli S, Oseid E et al (2013) Ebselen treatment prevents islet apoptosis, maintains intranuclear Pdx-1 and MafA levels, and preserves $\beta$-cell mass and function in ZDF rats. Diabetes $62(10): 3582-3588$

Mancini G, de Oliveira J, Hort MA et al (2014) Diphenyl diselenide differently modulates cardiovascular redox responses in young adult and middle-aged low-density lipoprotein receptor knockout hypercholesterolemic mice. J Pharm Pharmacol 66(3):387-397

Mao F, Chen J, Zhou Q, Luo Z, Huang L, Li X (2013) Novel tacrineebselen hybrids with improved cholinesterase inhibitory, hydrogen peroxide and peroxynitrite scavenging activity. Bioorg Med Chem Lett 23(24):6737-6742

Marins AT, Rodrigues CCR, de Menezes CC et al (2018) Integrated biomarkers response confirm the antioxidant role of diphenyl diselenide against atrazine. Ecotoxicol Environ Saf 151:191-198

Martin AL (1936) Toxicity of selenium to plants and animals. Am J Bot 471-483

Martini F, Augusto Bruning C, Mendonca Soares S, Wayne Nogueira C, Zeni G (2015) Inhibitory effect of ebselen on cerebral acetylcholinesterase activity in vitro: kinetics and reversibility of inhibition. Curr Pharm Des 21(7):920-924

Martini F, Pesarico AP, Brüning CA, Zeni G, Nogueira CW (2018) Ebselen inhibits the activity of acetylcholinesterase globular isoform G4 in vitro and attenuates scopolamine-induced amnesia in mice. J Cell Biochem 119(7):5598-5608

Martini F, Rosa SG, Klann IP et al (2019) A multifunctional compound ebselen reverses memory impairment, apoptosis and oxidative stress in a mouse model of sporadic Alzheimer's disease. J Psychiatr Res 109:107-117

Martins CC, Rosa SG, Recchi AM, Nogueira CW, Zeni G (2020) $\mathrm{m}$-Trifluoromethyl-diphenyl diselenide (m-CF3-PhSe) 2 modulates the hippocampal neurotoxic adaptations and abolishes a depressive-like phenotype in a short-term morphine withdrawal in mice. Prog Neuropsychopharmacol Biol Psychiatry 98:109803

Masaki C, Sharpley AL, Godlewska BR et al (2016b) Effects of the potential lithium-mimetic, ebselen, on brain neurochemistry: a magnetic resonance spectroscopy study at 7 tesla. Psychopharmacology 233(6):1097-1104

Masaki C, Sharpley AL, Cooper CM et al (2016a) Effects of the potential lithium-mimetic, ebselen, on impulsivity and emotional processing. Psychopharmacology 233(14):2655-2661

May HC, Yu J-J, Guentzel MN, Chambers JP, Cap AP, Arulanandam BP (2018) Repurposing auranofin, ebselen, and PX-12 as antimicrobial agents targeting the thioredoxin system. Front Microbiol 9:336

Mazzanti CM, Spanevello R, Ahmed M et al (2009) Pre-treatment with ebselen and vitamin $\mathrm{E}$ modulate acetylcholinesterase activity: interaction with demyelinating agents. Int J Dev Neurosci 27(1):73-80

McPhee DL, Janz DM (2014) Dietary selenomethionine exposure alters swimming performance, metabolic capacity and energy homeostasis in juvenile fathead minnow. Aquat Toxicol 155:91-100

Medeiros MC, Mello A, Gemelli T et al (2012) Effect of chronic administration of the vinyl chalcogenide 3-methyl-1-phenyl-2(phenylseleno) oct-2-en-1-one on oxidative stress in different brain areas of rats. Neurochem Res 37(5):928-934

Meinerz D, de Paula M, Comparsi B et al (2011) Protective effects of organoselenium compounds against methylmercury-induced oxidative stress in mouse brain mitochondrial-enriched fractions. Braz J Med Biol Res 44(11):1156-1163

Meinerz DF, Allebrandt J, Mariano DO et al (2014) Differential genotoxicity of diphenyl diselenide $(\mathrm{PhSe}) 2$ and diphenyl ditelluride (PhTe) 2. PeerJ 2:e290

Mello A, Medeiros MC, dos Santos LD et al (2012) Toxicological evaluation of chronic exposure to the organochalcogen 3-methyl1-phenyl-2-(phenylseleno) oct-2-en-1-one in male rats. Food Chem Toxicol 50(7):2450-2455

Melo M, De Oliveira I, Grivicich I et al (2013) Diphenyl diselenide protects cultured MCF-7 cells against tamoxifen-induced oxidative DNA damage. Biomed Pharmacother 67(4):329-335

Melo AM, Poester VR, Trapaga M et al (2020) Diphenyl diselenide and its interaction with antifungals against Aspergillus spp. Med Mycol. https://doi.org/10.1093/mmy/myaa072

Menendez CA, Bylehn F, Perez-Lemus GR, Alvarado W, de Pablo JJ (2020) Molecular characterization of ebselen binding activity to SARS-CoV-2 main protease. arXiv preprint arXiv:200509805

Menezes C, Leitemperger J, Toni C et al (2013) Comparative study on effects of dietary with diphenyl diselenide on oxidative stress in carp (Cyprinus carpio) and silver catfish (Rhamdia sp.) exposed to herbicide clomazone. Environ Toxicol Pharmacol 36(2):706-714

Menezes C, Leitemperger J, Santi A et al (2014) Evaluation of the effects induced by dietary diphenyl diselenide on common carp Cyprinus carpio. Fish Physiol Biochem 40(1):141-149

Menezes C, Marins A, Murussi C, Pretto A, Leitemperger J, Loro VL (2016) Effects of diphenyl diselenide on growth, oxidative damage, and antioxidant response in silver catfish. Sci Total Environ 542:231-237

Meotti F, Borges V, Zeni G, Rocha J, Nogueira C (2003) Potential renal and hepatic toxicity of diphenyl diselenide, diphenyl ditelluride and Ebselen for rats and mice. Toxicol Lett 143(1):9-16

Meotti FC, Coelho IS, Franco JL, Dafre AL, Rocha JB, Santos AR (2009) Redox modulation at the peripheral site alters nociceptive transmission in vivo. Clin Exp Pharmacol Physiol 36(3):272-277

Mertens K, Lowes D, Webster N et al (2015) Low zinc and selenium concentrations in sepsis are associated with oxidative damage and inflammation. BJA Br J Anaesth 114(6):990-999

Misu H, Ishikura K, Kurita S et al (2012) Inverse correlation between serum levels of selenoprotein $\mathrm{P}$ and adiponectin in patients with type 2 diabetes. PLoS ONE 7(4):e34952

Mix M, Singh AK, Tills M et al (2015b) Randomized phase II trial of selenomethionine as a modulator of efficacy and toxicity of 
chemoradiation in squamous cell carcinoma of the head and neck. World J Clin Oncol 6(5):166

Mix M, Ramnath N, Gomez J et al (2015a) Effects of selenomethionine on acute toxicities from concurrent chemoradiation for inoperable stage III non-small cell lung cancer. World J Clin Oncol 6(5): 156

Moghaddam A, Heller RA, Sun Q et al (2020) Selenium deficiency is associated with mortality risk from COVID-19. Nutrients 12(7):2098

Mordente A, Silvestrini A, Martorana GE, Tavian D, Meucci E (2015) Inhibition of anthracycline alcohol metabolite formation in human heart cytosol: a potential role for several promising drugs. Drug Metab Dispos 43(11):1691-1701

Muecke R, Micke O, Schomburg L et al (2014) Multicenter, phase III trial comparing selenium supplementation with observation in gynecologic radiation oncology: follow-up analysis of the survival data 6 years after cessation of randomization. Integr Cancer Ther 13(6):463-467

Mukherjee S, Weiner WS, Schroeder CE et al (2014) Ebselen inhibits hepatitis C virus NS3 helicase binding to nucleic acid and prevents viral replication. ACS Chem Biol 9(10):2393-2403

Müller A, Gabriel H, Sies H, Terlinden R, Fischer H, Römer A (1988) A novel biologically active selenooorganic compoundVII: biotransformation of ebselen in perfused rat liver. Biochem Pharmacol 37(6):1103-1109

Müller SG, Jardim NS, Quines CB, Nogueira CW (2018) Diphenyl diselenide regulates Nrf2/Keap-1 signaling pathway and counteracts hepatic oxidative stress induced by bisphenol A in male mice. Environ Res 164:280-287

Müller A, Cadenas E, Graf P, Sies H (1984) A Novel biologicallyactive organoselenium compound-1: glutathione peroxidaselike activity in vitro and antioxidant capacity of PZ-51 (Ebselen). Biochem Pharmacol 33:3235-3239

Naderi M, Salahinejad A, Jamwal A, Chivers DP, Niyogi S (2017) Chronic dietary selenomethionine exposure induces oxidative stress, dopaminergic dysfunction, and cognitive impairment in adult zebrafish (Danio rerio). Environ Sci Technol 51(21):12879-12888

Navarro-Alarcon M, Cabrera-Vique C (2008) Selenium in food and the human body: a review. Sci Total Environ 400(1-3):115-141

Navarro-Alarcon M, López-Martınez M (2000) Essentiality of selenium in the human body: relationship with different diseases. Sci Total Environ 249(1-3):347-371

Nayini J, El-Bayoumy K, Sugie S, Cohen LA, Reddy BS (1989) Chemoprevention of experimental mammary carcinogenesis by the synthetic organoselenium compound, benzylselenocyanate, in rats. Carcinogenesis 10(3):509-512

Nelson AA, Fitzhugh OG, Calvery HO (1943) Liver tumors following cirrhosis caused by selenium in rats. Can Res 3(4):230-236

Ngo HX, Shrestha SK, Garneau-Tsodikova S (2016) Identification of ebsulfur analogues with broad-spectrum antifungal activity. ChemMedChem 11(14):1507-1516

Nicastro HL, Dunn BK (2013) Selenium and prostate cancer prevention: insights from the selenium and vitamin E cancer prevention trial (SELECT). Nutrients 5(4):1122-1148

Ninomiya M, Garud DR, Koketsu M (2011) Biologically significant selenium-containing heterocycles. Coord Chem Rev 255(23-24):2968-2990

Nogara PA, Orian L, Rocha JBT (2020) The Se S/N interactions as a possible mechanism of $\delta$-aminolevulinic acid dehydratase enzyme inhibition by organoselenium compounds: a computational study. Comput Toxicol 15:100127

Nogueira CW, Rocha JB (2010) Diphenyl diselenide a janus-faced molecule. J Braz Chem Soc 21(11):2055-2071
Nogueira CW, Rocha JB (2011) Toxicology and pharmacology of selenium: emphasis on synthetic organoselenium compounds. Arch Toxicol 85(11):1313-1359

Nogueira CW, Rotta LN, Perry ML, Souza DO, da Rocha JBT (2001) Diphenyl diselenide and diphenyl ditelluride affect the rat glutamatergic system in vitro and in vivo. Brain Res 906(1-2):157-163

Nogueira C, Quinhones E, Jung E, Zeni G, Rocha J (2003) Antiinflammatory and antinociceptive activity of diphenyl diselenide. Inflamm Res 52(2):56-63

Nogueira CW, Zeni G, Rocha JB (2004) Organoselenium and organotellurium compounds: toxicology and pharmacology. Chem Rev 104(12):6255-6286

Nogueira CW, Borges LP, Souza ACG (2009) Oral administration of diphenyl diselenide potentiates hepatotoxicity induced by carbon tetrachloride in rats. J Appl Toxicol 29(2):156-164

Noiri E, Nakao A, Uchida K et al (2001) Oxidative and nitrosative stress in acute renal ischemia. Am J Physiol Ren Physiol 281:F948-F957

Occai BK, Hassan W, da Rocha JBT (2018) Gender-based behavioral and biochemical effects of diphenyl diselenide in Drosophila melanogaster. Chem Biol Interact 279:196-202

Ogawa A, Yoshimoto T, Kikuchi H et al (1999) Ebselen in acute middle cerebral artery occlusion: a placebo-controlled, double-blind clinical trial. Cerebrovasc Dis 9(2):112-118

Ohta Y, Kobayashi T, Inui K, Yoshino J, Nakazawa S (2002) Protective effect of ebselen, a seleno-organic compound, against the progression of acute gastric mucosal lesions induced by compound 48/80, a mast cell degranulator, in rats. Jpn J Pharmacol 90(4):295-303

Oldfield JE (1987) The two faces of selenium. J Nutr 117(12):2002-2008

Oliveira CES, Gai BM, Godoi B, Zeni G, Nogueira CW (2012) The antidepressant-like action of a simple selenium-containing molecule, methyl phenyl selenide, in mice. Eur J Pharmacol 690(1-3):119-123

Oliveira CES, Sari MHMM, Zborowski VA, Prado VC, Nogueira CW, Zeni G (2016) Pain-depression dyad induced by reserpine is relieved by $p, p^{\prime}$-methoxyl-diphenyl diselenide in rats. Eur $\mathbf{J}$ Pharmacol 791:794-802

Oliveira CES, Sari MHM, Zborowski VA, Araujo PCO, Nogueira CW, Zeni G (2017) $p, p^{\prime}$-Methoxyl-diphenyl diselenide elicits an antidepressant-like effect in mice without discontinuation anxiety phenotype. Pharmacol Biochem Behav 154:31-38

Oliveira DH, Sousa FS, Birmann PT, Alves D, Jacob RG, Savegnago L (2020) Antinociceptive and anti-inflammatory effects of 4-(arylchalcogenyl)-1H-pyrazoles containing selenium or sulfur. Pharmacological Reports 72(1):36-46

Olloquequi J, Cornejo-Córdova E, Verdaguer E et al (2018) Excitotoxicity in the pathogenesis of neurological and psychiatric disorders: therapeutic implications. J Psychopharmacol 32(3):265-275

Orian L, Toppo S (2014) Organochalcogen peroxidase mimetics as potential drugs: a long story of a promise still unfulfilled. Free Radic Biol Med 66:65-74

Orie NN, Warren AR, Basaric J et al (2017) In vitro assessment of the growth and plasma membrane H+-ATPase inhibitory activity of ebselen and structurally related selenium-and sulfur-containing compounds in Candida albicans. J Biochem Mol Toxicol 31(6):e21892

Orzáez M, Sancho M, Marchán S et al (2014) Apaf-1 inhibitors protect from unwanted cell death in in vivo models of kidney ischemia and chemotherapy induced ototoxicity. PLoS ONE 9(10):e110979

Oshita M, Takei Y, Kawano S, Fusamoto H, Kamada T (1994) Protective effect of ebselen on constrictive hepatic vasculature: 
prevention of alcohol-induced effects on portal pressure in perfused livers. J Pharmacol Exp Ther 271(1):20-24

Ozaki M, Nakamura M, Teraoka S, Ota K (1997) Ebselen, a novel anti-oxidant compound, protects the rat liver from ischemiareperfusion injury. Transpl Int 10(2):96-102

Ozgur T, Tutanc M, Zararsiz I et al (2012) The protective effect of ebselen on radiocontrast-induced nephrotoxicity. Ren Fail 34(8):991-997

Painter EP (1941) The chemistry and toxicity of selenium compounds, with special reference to the selenium problem. Chem Rev 28(2):179-213

Panchuk R, Skorokhyd N, Chumak V et al (2014) Specific antioxidant compounds differentially modulate cytotoxic activity of doxorubicin and cisplatin: in vitro and in vivo study. Croat Med $\mathbf{J}$ 55(3):206-217

Panchuk RR, Skorokhyd NR, Kozak S, Y, et al (2016) Antioxidants selenomethionine and D-pantethine decrease the negative side effects of doxorubicin in NL/Ly lymphoma-bearing mice. Croat Med J 57(2):180-192

Park S, Kang S, Kim D, Shin B, Moon N, Daily Iii J (2014) Ebselen pretreatment attenuates ischemia/reperfusion injury and prevents hyperglycemia by improving hepatic insulin signaling and $\beta$-cell survival in gerbils. Free Radic Res 48(8):864-874

Parnham MJ, Graf E (1987) Seleno-organic compounds and the therapy of hydroperoxide-linked pathological conditions. Biochem Pharmacol 36(19):3095-3102

Parnham MJ, Sies H (2013) The early research and development of ebselen. Biochem Pharmacol 86(9):1248-1253

Parnham M, Leyck S, Graf E, Dowling E, Blake D (1991) The pharmacology of ebselen. Agents Actions 32(1-2):4-9

Peglow TJ, Schumacher RF, Cargnelutti R et al (2017) Preparation of bis (2-pyridyl) diselenide derivatives: Synthesis of selenazolo [5, 4-b] pyridines and unsymmetrical diorganyl selenides, and evaluation of antioxidant and anticholinesterasic activities. Tetrahedron Lett 58(38):3734-3738

Pesarico AP, Sartori G, dos Santos CF et al (2013) 2, 2'-Dithienyl diselenide pro-oxidant activity accounts for antibacterial and antifungal activities. Microbiol Res 168(9):563-568

Petronilho F, Michels M, Danielski LG et al (2016) Diphenyl diselenide attenuates oxidative stress and inflammatory parameters in ulcerative colitis: a comparison with ebselen. Pathol Res Pract 212(9):755-760

Pettem CM, Weber LP, Janz DM (2017) Cardiac and metabolic effects of dietary selenomethionine exposure in adult zebrafish. Toxicol Sci 159(2):449-460

Phadnis PP, Hodage AS, Priyadarsini K, Jain VK (2014) Organoselenium compounds based on substituted acetanilides: synthesis. Charact Antioxid Act 53A:34-40

Pillaiyar T, Manickam M, Namasivayam V, Hayashi Y, Jung S-H (2016) An overview of severe acute respiratory syndrome-coronavirus (SARS-CoV) 3CL protease inhibitors: peptidomimetics and small molecule chemotherapy. J Med Chem 59(14):6595-6628

Pinto LG, Jesse CR, Nogueira CW, Savegnago L (2008) Evidence for the involvement of glutamatergic and GABAergic systems and protein kinase A pathway in the antinociceptive effect caused by $p$-methoxy-diphenyl diselenide in mice. Pharmacol Biochem Behav 88(4):487-496

Pinto JT, Krasnikov BF, Alcutt S et al (2014) Kynurenine aminotransferase III and glutamine transaminase L are identical enzymes that have cysteine $S$-conjugate $\beta$-lyase activity and can transaminate L-selenomethionine. J Biol Chem 289(45):30950-30961

Pinton S, da Rocha JT, Zeni G, Nogueira CW (2010) Organoselenium improves memory decline in mice: involvement of acetylcholinesterase activity. Neurosci Lett 472(1):56-60

Pinton S, da Rocha JT, Gai BM, Prigol M, da Rosa LV, Nogueira CW (2011) Neuroprotector effect of $p, p$ '-methoxyl-diphenyl diselenide in a model of sporadic dementia of Alzheimer's type in mice: contribution of antioxidant mechanism. Cell Biochem Funct 29(3):235-243

Pinton S, Sampaio TB, Ramalho RM, Rodrigues CM, Nogueira CW (2013b) $p, p^{\prime}$-Methoxyl-diphenyl diselenide prevents neurodegeneration and glial cell activation induced by streptozotocin in rats. J Alzheimer's Dis 33(1):133-144

Pinton S, Brüning CA, Oliveira CES, Prigol M, Nogueira CW (2013a) Therapeutic effect of organoselenium dietary supplementation in a sporadic dementia of Alzheimer's type model in rats. J Nutr Biochemis 24(1):311-317

Pinton S, Souza AC, Sari MH, Ramalho RM, Rodrigues CM, Nogueira CW (2013c) p,p'-Methoxyl-diphenyl diselenide protects against amyloid- $\beta$ induced cytotoxicity in vitro and improves memory deficits in vivo. Behav Brain Res 247:241-247

Pinz M, Reis AS, Duarte V et al (2016) 4-Phenylselenyl-7-chloroquinoline, a new quinoline derivative containing selenium, has potential antinociceptive and anti-inflammatory actions. Eur J Pharmacol 780:122-128

Pitts MW, Hoffmann PR (2018) Endoplasmic reticulum-resident selenoproteins as regulators of calcium signaling and homeostasis. Cell Calcium 70:76-86

Pivetta LA, Pereira RP, Farinon M et al (2006) Ethanol inhibits $\delta$-aminolevulinate dehydratase and glutathione peroxidase activities in mice liver: protective effects of ebselen and $N$-acetylcysteine. Environ Toxicol Pharmacol 21(3):338-343

Poester VR, Mattei AS, Mendes JF et al (2019) Antifungal activity of diphenyl diselenide alone and in combination with itraconazole against Sporothrix brasiliensis. Med Mycol 57(3):328-331

Pons DG, Moran C, Alorda-Clara M, Oliver J, Roca P, Sastre-Serra J (2020) Micronutrients selenomethionine and selenocysteine modulate the redox status of MCF-7 breast cancer cells. Nutrients 12(3):865

Posser T, Franco JL, dos Santos DA et al (2008) Diphenyl diselenide confers neuroprotection against hydrogen peroxide toxicity in hippocampal slices. Brain Res 1199:138-147

Posser T, Kaster MP, Baraúna SC, Rocha JB, Rodrigues ALS, Leal RB (2009) Antidepressant-like effect of the organoselenium compound ebselen in mice: evidence for the involvement of the monoaminergic system. Eur J Pharmacol 602(1):85-91

Posser T, de Paula MT, Franco JL, Leal RB, da Rocha JBT (2011) Diphenyl diselenide induces apoptotic cell death and modulates ERK1/2 phosphorylation in human neuroblastoma SHSY5Y cells. Arch Toxicol 85(6):645-651

Pothion H, Jehan C, Tostivint $\mathrm{H}$ et al (2020) Selenoprotein T: an essential oxidoreductase serving as a guardian of endoplasmic reticulum homeostasis. Antioxid Redox Signal 33(17):1257-1275

Prabhu CP, Phadnis PP, Wadawale AP, Priyadarsini KI, Jain VK (2012) Synthesis, characterization, structures and antioxidant activity of nicotinoyl based organoselenium compounds. J Organomet Chem 713:42-50

Prauchner CA, Prestes AdS, da Rocha JB (2011) Effects of diphenyl diselenide on oxidative stress induced by sepsis in rats. Pathol Res Pract 207(9):554-558

Prigol M, Wilhelm EA, Schneider CC, Rocha JB, Nogueira CW, Zeni $G$ (2007) Involvement of oxidative stress in seizures induced by diphenyl diselenide in rat pups. Brain Res 1147:226-232

Prigol M, Luchese C, Pinton S et al (2011) Diphenyl diselenide induces anxiolytic-like and sedative effects on the chick social separation-stress behavior. Neurosci Lett 495(2):140-143

Prigol M, Nogueira CW, Zeni G, Bronze MR, Constantino L (2012) In vitro metabolism of diphenyl diselenide in rat liver fractions conjugation with GSH and binding to thiol groups. Chem Biol Interact 200(2-3):65-72 
Prigol M, Nogueira CW, Zeni G, Bronze MR, Constantino L (2013) Physicochemical and biochemical profiling of diphenyl diselenide. Appl Biochem Biotechnol 169(3):885-893

Puntel RL, Roos DH, Folmer V et al (2010) Mitochondrial dysfunction induced by different organochalchogens is mediated by thiol oxidation and is not dependent of the classical mitochondrial permeability transition pore opening. Toxicol Sci 117(1):133-143

Puntel RL, Roos DH, Seeger RL, Aschner M, Rocha JBT (2013) Organochalcogens inhibit mitochondrial complexes I and II in rat brain: possible implications for neurotoxicity. Neurotox Res 24(2):109-118

Qian F, Misra S, Prabhu KS (2019) Selenium and selenoproteins in prostanoid metabolism and immunity. Crit Rev Biochem Mol Biol 54(6):484-516

Qiu-Ju L, Bessems JG, Commandeur JN, Adams B, Vermeulen NP (1994) Mechanism of protection of ebselen against paracetamol-induced toxicity in rat hepatocytes. Biochem Pharmacol 48(8):1631-1640

Qu J, Wang W, Zhang Q, Li S (2020) Inhibition of lipopolysaccharideinduced inflammation of chicken liver tissue by selenomethionine via TLR4-NF-кB-NLRP3 signaling pathway. Biol Trace Elem Res 195:205-214

Quines CB, Rosa SG, Velasquez D, Da Rocha JT, Neto JS, Nogueira CW (2016b) Diphenyl diselenide elicits antidepressant-like activity in rats exposed to monosodium glutamate: a contribution of serotonin uptake and $\mathrm{Na}+, \mathrm{K}+$-ATPase activity. Behav Brain Res 301:161-167

Quines CB, Rosa SG, Chagas PM et al (2016a) Homeostatic effect of p-chloro-diphenyl diselenide on glucose metabolism and mitochondrial function alterations induced by monosodium glutamate administration to rats. Amino Acids 48(1):137-148

Quines CB, Chagas PM, Hartmann D, Carvalho NR, Soares FA, Nogueira CW (2017a) (p-ClPhSe) 2 reduces hepatotoxicity induced by monosodium glutamate by improving mitochondrial function in rats. J Cell Biochem 118(9):2877-2886

Quines CB, Rosa SG, Chagas PM, Velasquez D, Prado VC, Nogueira $\mathrm{CW}$ (2017b) (p-ClPhSe) 2 stimulates carbohydrate metabolism and reverses the metabolic alterations induced by high fructose load in rats. Food Chem Toxicol 107:122-128

Quines CB, Rosa SG, Velasquez D, Prado VC, Neto JS, Nogueira CW (2018) (p-ClPhSe) 2 stabilizes metabolic function in a rat model of neuroendocrine obesity induced by monosodium glutamate. Food Chem Toxicol 118:168-180

Quispe RL, Jaramillo ML, Galant LS et al (2019) Diphenyl diselenide protects neuronal cells against oxidative stress and mitochondrial dysfunction: involvement of the glutathione-dependent antioxidant system. Redox Biol 20:118-129

Ramalho JB, Izaguirry AP, Soares MB et al (2018) Selenofuranoside improves long-term memory deficits in rats after exposure to monosodium glutamate: involvement of $\mathrm{Na}+, \mathrm{K}+$-ATPase activity. Physiol Behav 184:27-33

Rayman MP (2000) The importance of selenium to human health. Lancet 356(9225):233-241

Rayman MP (2012) Selenium and human health. Lancet 379(9822):1256-1268

Rayman MP (2020) Selenium intake, status, and health: a complex relationship. Hormones 19(1):9-14

Rayman MP, Stranges S (2013) Epidemiology of selenium and type 2 diabetes: can we make sense of it? Free Radic Biol Med 65:1557-1564

Rayman MP, Blundell-Pound G, Pastor-Barriuso R, Guallar E, Steinbrenner H, Stranges S (2012) A randomized trial of selenium supplementation and risk of type-2 diabetes, as assessed by plasma adiponectin. PLoS ONE 7(9):e45269
Rea HM, Thomson CD, Campbell DR, Robinson MF (1979) Relation between erythrocyte selenium concentrations and glutathione peroxidase (EC 1.11. 1.9) activities of New Zealand residents and visitors to New Zealand. Br J Nutr 42(2):201-208

Reddy B, Tanaka T, Simi B (1985) Effect of different levels of dietary trans fat or corn oil on azoxymethane-induced colon carcinogenesis in F344 rats. J Natl Cancer Inst 75(4):791-798

Reddy BS, Sugie S, Maruyama H, El-Bayoumy K, Marra P (1987) Chemoprevention of colon carcinogenesis by dietary organoselenium, benzylselenocyanate, in F344 rats. Can Res 47(22):5901-5904

Reddy B, Hirose Y, Lubet R, Steele V, Kelloff G, Rao C (2000) Lack of chemopreventive efficacy of DL-selenomethionine in colon carcinogenesis. Int J Mol Med 5(4):327-357

Reddy VS, Bukke S, Dutt N, Rana P, Pandey AK (2017) A systematic review and meta-analysis of the circulatory, erythrocellular and CSF selenium levels in Alzheimer's disease: a metal metaanalysis (AMMA study-I). J Trace Elem Med Biol 42:68-75

Reis AS, Pinz MP, Bortolatto CF et al (2017b) Antioxidant compound (E)-2-benzylidene-4-phenyl-1, 3-diselenole protects rats against thioacetamide-induced acute hepatotoxicity. Can J Physiol Pharmacol 95(9):1039-1045

Reis AS, Pinz M, Duarte LFB et al (2017a) 4-phenylselenyl-7-chloroquinoline, a novel multitarget compound with anxiolytic activity: contribution of the glutamatergic system. J Psychiatr Res 84:191-199

Reis AS, Vogt AG, Pinz MP et al (2019) Modulation of COX-2, INF- $y$, glutamatergic and opioid systems contributes to antinociceptive, anti-inflammatory and anti-hyperalgesic effects of bis (3-amino-2-pyridine) diselenide. Chem Biol Interact 311:108790

Reyes L, Bishop DP, Hawkins CL, Rayner BS (2019) Assessing the efficacy of dietary selenomethionine supplementation in the setting of cardiac ischemia/reperfusion injury. Antioxidants 8(11):546

Ribeiro MCP, Ávila DS, Schiar VPP et al (2013) Diphenyl diselenide supplementation reduces biochemical alterations associated with oxidative stress in rats fed with fructose and hydrochlorothiazide. Chem Biol Interact 204(3):191-199

Rocca C, Boukhzar L, Granieri M et al (2018) A selenoprotein T-derived peptide protects the heart against ischaemia/reperfusion injury through inhibition of apoptosis and oxidative stress. Acta Physiol 223(4):e13067

Rocha J, Gabriel D, Zeni G et al (2005) Ebselen and diphenyl diselenide change biochemical hepatic responses to overdosage with paracetamol. Environ Toxicol Pharmacol 19(2):255-261

Rocha JB, Saraiva RA, Garcia SC, Gravina FS, Nogueira CW (2012c) Aminolevulinate dehydratase ( $\delta$-ALA-D) as marker protein of intoxication with metals and other pro-oxidant situations. Toxicol Res 1(2):85-102

Rocha JB, Piccoli BC, Oliveira CS (2017) Biological and chemical interest in selenium: a brief historical account. ARKIVOC Online J Organ Chem 2017:457-491

Rocourt CR, Cheng W-H (2013) Selenium supranutrition: are the potential benefits of chemoprevention outweighed by the promotion of diabetes and insulin resistance? Nutrients 5(4):1349-1365

Rodríguez-Sosa M, García-Montalvo EA, Del Razo LM, Vega L (2013) Effect of selenomethionine supplementation in food on the excretion and toxicity of arsenic exposure in female mice. Biol Trace Elem Res 156(1-3):279-287

Rosa RM, Flores DG, Appelt HR, Braga AL, Henriques JAP, Roesler R (2003) Facilitation of long-term object recognition memory by pretraining administration of diphenyl diselenide in mice. Neurosci Lett 341(3):217-220

Rosa SG, Quines CB, da Rocha JT, Bortolatto CF, Duarte T, Nogueira CW (2015) Antinociceptive action of diphenyl diselenide in the 
nociception induced by neonatal administration of monosodium glutamate in rats. Eur J Pharmacol 758:64-71

Rosa SG, Quines CB, Stangherlin EC, Nogueira CW (2016) Diphenyl diselenide ameliorates monosodium glutamate induced anxietylike behavior in rats by modulating hippocampal BDNF-Akt pathway and uptake of GABA and serotonin neurotransmitters. Physiol Behav 155:1-8

Rosa SG, Pesarico AP, Tagliapietra CF, da Luz SC, Nogueira CW (2017) Opioid system contribution to the antidepressant-like action of m-trifluoromethyl-diphenyl diselenide in mice: a compound devoid of tolerance and withdrawal syndrome. J Psychopharmacol 31(9):1250-1262

Rosa SG, Brüning CA, Pesarico AP, de Souza ACG, Nogueira CW (2018a) Anti-inflammatory and antinociceptive effects of 2, 2-dipyridyl diselenide through reduction of inducible nitric oxide synthase, nuclear factor-kappa B and c-Jun N-terminal kinase phosphorylation levels in the mouse spinal cord. J Trace Elem Med Biol 48:38-45

Rosa SG, Pesarico AP, Martini F, Nogueira CW (2018b) m-Trifluoromethyl-diphenyl diselenide regulates prefrontal cortical MOR and KOR protein levels and abolishes the phenotype induced by repeated forced swim stress in mice. Mol Neurobiol 55(12):8991-9000

Rosa SG, Pesarico AP, Nogueira CW (2018c) m-Trifluoromethyldiphenyl diselenide promotes resilience to social avoidance induced by social defeat stress in mice: contribution of opioid receptors and MAPKs. Prog Neuropsychopharmacol Biol Psychiatry $82: 123-135$

Roser M, Ritchie H, Ortiz-Ospina E, Hasell J (2020) Coronavirus pandemic (COVID-19). Our World Data. https://ourworldindata.org/ coronavirus/country/kazakhstan

Rossato L, Loreto ES, Venturini TP et al (2019) In vitro combination between antifungals and diphenyl diselenide against Cryptococcus species. Mycoses 62(6):508-512

Rosseti IB, Rocha JBT, Costa MS (2015) Diphenyl diselenide (PhSe) 2 inhibits biofilm formation by Candida albicans, increasing both ROS production and membrane permeability. J Trace Elem Med Biol 29:289-295

Rotruck JT, Pope AL, Ganther HE, Swanson A, Hafeman DG, Hoekstra W (1973) Selenium: biochemical role as a component of glutathione peroxidase. Science 179(4073):588-590

Ruberte AC, Sanmartin C, Aydillo C, Sharma AK, Plano D (2019) Development and therapeutic potential of selenazo compounds. J Med Chem 63(4):1473-1489

Rupil LL, de Bem AF, Roth GA (2012) Diphenyl diselenide-modulation of macrophage activation: down-regulation of classical and alternative activation markers. Innate Immunity 18(4):627-637

Saad SY, Najjar TA, Arafah MM (2006) Cardioprotective effects of subcutaneous ebselen against daunorubicin-induced cardiomyopathy in rats. Basic Clin Pharmacol Toxicol 99(6):412-417

Saeed A, Larik AF, Channar AP (2016) Synthetic approaches to the multifunctional drug ebselen and analogs: past and present. Mini-Rev Organ Chem 13(4):312-324

Safayhi H, Tiegs G, Wendel A (1985) A novel biologically active seleno-organic compound-V: Inhibition by ebselen (PZ 51) of rat peritoneal neutrophil lipoxygenase. Biochem Pharmacol 34(15):2691-2694

Saito I, Asano T, Sano K et al (1998) Neuroprotective effect of an antioxidant, ebselen, in patients with delayed neurological deficits after aneurysmal subarachnoid hemorrhage. Neurosurgery 42(2):269-277

Sampaio TB, Da Rocha JT, Prigol M et al (2016) 4-Organoselenoisoquinolines selectively and reversibly inhibit the cerebral monoamine oxidase B activity. J Mol Neurosci 59(1):135-145

Sampaio TB, da Rocha JT, Quines CB, Stein ALA, Zeni G, Nogueira CW (2017a) Sulfhydryl-based inhibition of $\delta$-ALA-D and Na+,
$\mathrm{K}+$-ATPase activities depends on the organoselenium group bonded to the isoquinoline. J Cell Biochem 118(5):1144-1150

Sampaio TB, Pinton S, da Rocha JT, Gai BM, Nogueira CW (2017b) Involvement of BDNF/TrkB signaling in the effect of diphenyl diselenide on motor function in a Parkinson's disease rat model. Eur J Pharmacol 795:28-35

Sampaio TB, Bilheri FN, Zeni GR, Nogueira CW (2020) Dopaminergic system contribution to the antidepressant-like effect of 3-phenyl-4-(phenylseleno) isoquinoline in mice. Behav Brain Res 386:112602

Sancineto L, Piccioni M, De Marco S et al (2016) Diphenyl diselenide derivatives inhibit microbial biofilm formation involved in wound infection. BMC Microbiol 16(1):220

Sands KN, Tuck TA, Back TG (2018) Cyclic seleninate esters, spirodioxyselenuranes and related compounds: new classes of biological antioxidants that emulate glutathione peroxidase. Chem A Eur J 24(39):9714-9728

Sanmartín C, Plano D, Sharma AK, Palop JA (2012) Selenium compounds, apoptosis and other types of cell death: an overview for cancer therapy. Int J Mol Sci 13(8):9649-9672

Santofimia-Castano P, Salido GM, González A (2013) Ebselen alters mitochondrial physiology and reduces viability of rat hippocampal astrocytes. DNA Cell Biol 32(4):147-155

Santofimia-Castaño P, Izquierdo-Alvarez A, de la Casa-Resino I et al (2016) Ebselen alters cellular oxidative status and induces endoplasmic reticulum stress in rat hippocampal astrocytes. Toxicology 357:74-84

Santofimia-Castaño P, Izquierdo-Alvarez A, Plaza-Davila M et al (2018) Ebselen impairs cellular oxidative state and induces endoplasmic reticulum stress and activation of crucial mitogenactivated protein kinases in pancreatic tumour AR42J cells. J Cell Biochem 119(1):1122-1133

Santos FW, Zeni G, Rocha JB et al (2005b) Diphenyl diselenide reverses cadmium-induced oxidative damage on mice tissues. Chem Biol Interact 151(3):159-165

Santos FW, Zeni G, Rocha JB, do Nascimento PC, Marques MS, Nogueira CW (2005a) Efficacy of 2, 3-dimercapto-1-propanesulfonic acid (DMPS) and diphenyl diselenide on cadmium induced testicular damage in mice. Food Chem Toxicol 43(12): 1723-1730

Saraiva R, Bueno D, Nogara P, Rocha J (2012) Molecular docking studies of disubstituted diaryl diselenides as mammalian $\delta$-aminolevulinic acid dehydratase enzyme inhibitors. J Toxicol Environ Health Part A 75(16-17):1012-1022

Sareila O, Kelkka T, Pizzolla A, Hultqvist M, Holmdahl R (2011) Nox2 complex-derived ROS as immune regulators. Antioxid Redox Signal 15(8):2197-2208

Sari MHM, Souza ACG, Rosa SG, Souza D, Rodrigues OED, Nogueira CW (2014) Contribution of dopaminergic and adenosinergic systems in the antinociceptive effect of p-chloro-selenosteroid. Eur J Pharmacol 725:79-86

Sari MHM, Ferreira LM, Zborowski VA et al (2017) p, p'-Methoxyldiphenyl diselenide-loaded polymeric nanocapsules are chemically stable and do not induce toxicity in mice. Eur J Pharm Biopharm 117:39-48

Sari MHM, Zborowski VA, Ferreira LM et al (2018b) p, p'-Methoxyldiphenyl diselenide-loaded polymeric nanocapsules as a novel approach to inflammatory pain treatment: behavioral, biochemistry and molecular evidence. Eur J Pharm Sci 111:38-45

Sari MHM, Zborowski VA, Ferreira LM et al (2018a) Enhanced pharmacological actions of p, p'-methoxyl-diphenyl diselenideloaded polymeric nanocapsules in a mouse model of neuropathic pain: behavioral and molecular insights. J Trace Elem Med Biol 46:17-25

Sarma BK, Mugesh G (2008) Thiol cofactors for selenoenzymes and their synthetic mimics. Org Biomol Chem 6(6):965-974 
Sartori G, Jardim NS, Marcondes Sari MH et al (2016) Antiviral action of diphenyl diselenide on herpes simplex virus 2 infection in female BALB/c mice. J Cell Biochem 117(7):1638-1648

Sartori G, Jardim NS, Sari MHM, Flores EF, Prigol M, Nogueira CW (2017) Diphenyl diselenide reduces oxidative stress and toxicity caused by HSV-2 infection in mice. J Cell Biochem 118(5):1028-1037

Sartori Oliveira CE, Pinton S, da Rocha JT, Gai BM, Nogueira CW (2016) The hypolipidemic action of a diet supplemented with $p, p^{\prime}$-methoxyl-diphenyl diselenide is not directly related to its antioxidant property. Can J Physiol Pharmacol 94(6):662-668

Sasaki M, Fujimoto S, Sato Y et al (2013) Reduction of reactive oxygen species ameliorates metabolism-secretion coupling in islets of diabetic GK rats by suppressing lactate overproduction. Diabetes 62(6):1996-2003

Sauer AC, Leal JG, Stefanello ST et al (2017) Synthesis and antioxidant properties of organosulfur and organoselenium compounds derived from 5-substituted-1, 3, 4-oxadiazole/thiadiazole-2-thiols. Tetrahedron Lett 58(1):87-91

Savegnago L, Trevisan M, Alves D, Rocha JBT, Nogueira CW, Zeni G (2006b) Antisecretory and antiulcer effects of diphenyl diselenide. Environ Toxicol Pharmacol 21(1):86-92

Savegnago L, Jesse CR, Moro AV et al (2006a) Bis selenide alkene derivatives: a class of potential antioxidant and antinociceptive agents. Pharmacol Biochem Behav 83(2):221-229

Savegnago L, Pinto LG, Jesse CR et al (2007c) Antinociceptive properties of diphenyl diselenide: evidences for the mechanism of action. Eur J Pharmacol 555(2-3):129-138

Savegnago L, Jesse CR, Pinto LG, Rocha JB, Nogueira CW, Zeni G (2007b) Monoaminergic agents modulate antidepressant-like effect caused by diphenyl diselenide in rats. Prog Neuropsychopharmacol Biol Psychiatry 31(6):1261-1269

Savegnago L, Jesse CR, Pinto LG, Rocha JB, Nogueira CW (2007a) Diphenyl diselenide attenuates acute thermal hyperalgesia and persistent inflammatory and neuropathic pain behavior in mice. Brain Res 1175:54-59

Savegnago L, Jesse CR, Nogueira CW (2008a) Caffeine and a selective adenosine A2B receptor antagonist but not imidazoline receptor antagonists modulate antinociception induced by diphenyl diselenide in mice. Neurosci Lett 436(2):120-123

Savegnago L, Jesse CR, Pinto LG et al (2008b) Diphenyl diselenide exerts antidepressant-like and anxiolytic-like effects in mice: involvement of L-arginine-nitric oxide-soluble guanylate cyclase pathway in its antidepressant-like action. Pharmacol Biochem Behav 88(4):418-426

Savegnago L, Jesse CR, Nogueira CW (2009) Structural modifications into diphenyl diselenide molecule do not cause toxicity in mice. Environ Toxicol Pharmacol 27(2):271-276

Schewe T (1995) Molecular actions of ebselen - an antiinflammatory antioxidant. Gen Pharmacol Vasc Syst 26(6):1153-1169

Schewe C, Schewe T, Wendel A (1994) Strong inhibition of mammalian lipoxygenases by the antiinflammatory seleno-organic compound ebselen in the absence of glutathione. Biochem Pharmacol 48(1):65-74

Schomburg L (2020) The other view: The trace element selenium as a micronutrient in thyroid disease, diabetes, and beyond. Hormones 19:15-24

Schrauzer GN (2003) The nutritional significance, metabolism and toxicology of selenomethionine. Adv Food Nutr Res 47:73-112

Schwarz K, Foltz CM (1957) Selenium as an integral part of factor 3 against dietary necrotic liver degeneration. J Am Chem Soc 79(12):3292-3293

Schwarz K, Fredga A (1969) Biological potency of organic selenium compounds I. Aliphatic monoseleno-and diseleno-dicarboxylic acids. J Biol Chem 244(8):2103-2110
Seko Y, Saito Y, Kitahara J, Imura N (1989) Active oxygen generation by the reaction of selenite with reduced glutathione in vitro Selenium in biology and medicine. Springer, Berlin, pp 70-73

Selvakumar K, Shah P, Singh HB, Butcher RJ (2011) Synthesis, structure, and glutathione peroxidase-like activity of amino acid containing ebselen analogues and diaryl diselenides. Chem A Eur J 17(45):12741-12755

Serrão VHB, Silva IR, da Silva MTA, Scortecci JF, de Freitas FA, Thiemann OH (2018) The unique tRNA Sec and its role in selenocysteine biosynthesis. Amino Acids 50(9):1145-1167

Shaaban S, Negm A, Sobh MA, Wessjohann LA (2015) Organoselenocyanates and symmetrical diselenides redox modulators: design, synthesis and biological evaluation. Eur J Med Chem 97:190-201

Shamberger R, Frost D (1969) Possible protective effect of selenium against human cancer. Can Med Assoc J 100(14):682

Sharma AK, Amin S (2013) Post SELECT: selenium on trial. Fut Med Chem 5(2):163-174

Sharma P, Srivastava P, Seth A, Tripathi PN, Banerjee AG, Shrivastava SK (2019) Comprehensive review of mechanisms of pathogenesis involved in Alzheimer's disease and potential therapeutic strategies. Prog Neurobiol 174:53-89

Sharpley AL, Williams C, Holder AA et al (2020) A phase 2a randomised, double-blind, placebo-controlled, parallel-group, addon clinical trial of ebselen (SPI-1005) as a novel treatment for mania or hypomania. Psychopharmacology 237(12):3773-3782

Shchedrina VA, Zhang Y, Labunskyy VM, Hatfield DL, Gladyshev VN (2010) Structure-function relations, physiological roles, and evolution of mammalian ER-resident selenoproteins. Antioxid Redox Signal 12(7):839-849

Shen L, Shin K-M, Lee K-T, Jeong J-H (2004) Synthesis of new diselenide compounds as anti-inflammatory agents. Arch Pharmacal Res 27(8):816-819

Shi X, Wang W, Zheng S, Zhang Q, Xu S (2020) Selenomethionine relieves inflammation in the chicken trachea caused by LPS though inhibiting the NF- $\kappa \mathrm{B}$ pathway. Biol Trace Elem Res 194(2):525-535

Shimohashi N, Nakamuta M, Uchimura K et al (2000) Selenoorganic compound, ebselen, inhibits nitric oxide and tumor necrosis factor- $\alpha$ production by the modulation of Jun-N-terminal kinase and the NF-kb signaling pathway in rat Kupffer cells. J Cell Biochem 78(4):595-606

Shin KM, Shen L, Park SJ, Jeong JH, Lee KT (2009) Bis-(3-hydroxyphenyl) diselenide inhibits LPS-stimulated iNOS and COX-2 expression in RAW 2647 macrophage cells through the NF-kB inactivation. J Pharm Pharmacol 61(4):479-486

Shini S, Sultan A, Bryden WL (2015) Selenium biochemistry and bioavailability: Implications for animal agriculture. Agriculture 5(4): 1277-1288

Shivakoti R, Gupte N, Yang W-T et al (2014) Pre-antiretroviral therapy serum selenium concentrations predict WHO stages 3, 4 or death but not virologic failure post-antiretroviral therapy. Nutrients 6(11):5061-5078

Sies H (1994) [47] Ebselen: A glutathione peroxidase mimic. Methods Enzymol 234:476-482

Sies H, Parnham MJ (2020) Potential therapeutic use of ebselen for COVID-19 and other respiratory viral infections. Free Radic Biol Med

Silvestre F, Danielski LG, Michels M et al (2014) Effects of organoselenium compounds on early and late brain biochemical alterations in sepsis-survivor rats. Neurotox Res 26(4):382-391

Simonović M (1862) Puppala AK (2018) On elongation factor eEF$\mathrm{Sec}$, its role and mechanism during selenium incorporation into nascent selenoproteins. Biochim Biophys Acta (BBA) Gen Subj 11:2463-2472 
Singh N, Halliday AC, Thomas JM et al (2013) A safe lithium mimetic for bipolar disorder. Nat Commun 4(1):1-7

Singh VP, Jf P, Butcher RJ, Engman L (2014) Pyridoxine-derived organoselenium compounds with glutathione peroxidaselike and chain-breaking antioxidant activity. Chem A Eur J 20(39):12563-12571

Singh VP, Poon J-f, Butcher RJ et al (2015) Effect of a bromo substituent on the glutathione peroxidase activity of a pyridoxine-like diselenide. J Organ Chem 80(15):7385-7395

Singh N, Sharpley AL, Emir UE et al (2016) Effect of the putative lithium mimetic ebselen on brain myo-inositol, sleep, and emotional processing in humans. Neuropsychopharmacology 41(7): $1768-1778$

Singh BG, Kumar P, Phadnis P, Iwaoka M, Priyadarsini KI (2019) Free radical induced selenoxide formation in isomeric organoselenium compounds: the effect of chemical structures on antioxidant activity. New J Chem 43(34):13357-13362

Sinha R, Medina D (1997) Inhibition of cdk2 kinase activity by methylselenocysteine in synchronized mouse mammary epithelial tumor cells. Carcinogenesis 18(8):1541-1547

Sinha R, Kiley S, Lu J et al (1999) Effects of methylselenocysteine on PKC activity, cdk2 phosphorylation and gadd gene expression in synchronized mouse mammary epithelial tumor cells. Cancer Lett 146(2):135-145

Ślusarczyk W, Olakowska E, Larysz-Brysz M et al (2019) Use of ebselen as a neuroprotective agent in rat spinal cord subjected to traumatic injury. Neural Regen Res 14(7):1255

Smith M (1941) Chronic endemic selenium poisoning: a review of the more recent field and laboratory studies. J Am Med Assoc 116(7):562-567

Smith SM, Min J, Ganesh T et al (2012) Ebselen and congeners inhibit NADPH oxidase 2-dependent superoxide generation by interrupting the binding of regulatory subunits. Chem Biol 19(6):752-763

Soares JC, Folmer V, Da Rocha JB, Nogueira CW (2014) Ebselen exhibits glycation-inhibiting properties and protects against osmotic fragility of human erythrocytes in vitro. Cell Biol Int 38(5):625-630

Song G, Zhang Z, Wen L et al (2014) Selenomethionine ameliorates cognitive decline, reduces tau hyperphosphorylation, and reverses synaptic deficit in the triple transgenic mouse model of Alzheimer's disease. J Alzheimer's Dis 41(1):85-99

Souza ACG, Brüning CA, Leite MR, Zeni G, Nogueira CW (2010) Diphenyl diselenide improves scopolamine-induced memory impairment in mice. Behav Pharmacol 21(5-6):556-562

Souza CF, Baldissera MD, Descovi SN et al (2019) Diphenyl diselenide dietary supplementation protects against methylmercury-chloride-induced immunotoxicity in the head kidney and spleen of grass carp (Ctenopharyngodon idella) via regulation of purinergic signaling and the NLRP3 inflammasome. Comp Biochem Physiol C Toxicol Pharmacol 222:59-64

Soyman Z, Uzun H, Bayindir N, Esrefoglu M, Boran B (2018) Can ebselen prevent cisplatin-induced ovarian damage? Arch Gynecol Obstet 297(6):1549-1555

Spallholz JE (1994) On the nature of selenium toxicity and carcinostatic activity. Free Radic Biol Med 17(1):45-64

Spengler G, Gajdács M, Marć MA, Domínguez-Álvarez E, Sanmartín C (2019) Organoselenium compounds as novel adjuvants of chemotherapy drugs - a promising approach to fight cancer drug resistance. Molecules 24(2):336

Spiller HA, Shelton JP, Funk AR (2020) Fatal occupational selenomethionine poisoning with hazmat response: a case report. Toxicol Commun 4(1):12-14

Stangherlin EC, Luchese C, Pinton S, Rocha JBT, Nogueira CW (2008) Sub-chronical exposure to diphenyl diselenide enhances acquisition and retention of spatial memory in rats. Brain Res 1201:106-113

Stapleton S (2000) Selenium: an insulin mimetic. Cell Mol Life Sci CMLS 57(13-14):1874-1879

Stefanello ST, Flores da Rosa EJ, Dobrachinski F et al (2015) Effect of diselenide administration in thioacetamide-induced acute neurological and hepatic failure in mice. Toxicol Res 4(3):707-717

Stefanello ST, Dobrachinski F, de Carvalho NR et al (2015) Free radical scavenging in vitro and biological activity of diphenyl diselenide-loaded nanocapsules: DPDS-NCS Antioxidant and toxicological effects. Int J Nanomed 10:5663

Stefanello ST, Mizdal CR, Gonçalves DF et al (2020) The insertion of functional groups in organic selenium compounds promote changes in mitochondrial parameters and raise the antibacterial activity. Bioorgan Chem 98:103727

Steinbrenner H, Speckmann B, Sies H (2013) Toward understanding success and failures in the use of selenium for cancer prevention. Antioxid Redox Signal 19(2):181-191

Steinbrenner H, Al-Quraishy S, Dkhil MA, Wunderlich F, Sies H (2015) Dietary selenium in adjuvant therapy of viral and bacterial infections. Adv Nutr 6(1):73-82

Steinbrenner H, Bilgic E, Pinto A et al (2016) Selenium pretreatment for mitigation of ischemia/reperfusion injury in cardiovascular surgery: influence on acute organ damage and inflammatory response. Inflammation 39(4):1363-1376

Stolwijk JM, Garje R, Sieren JC, Buettner GR, Zakharia Y (2020) Understanding the redox biology of selenium in the search of targeted cancer therapies. Antioxidants 9(5):420

Stone CA, Kawai K, Kupka R, Fawzi WW (2010) Role of selenium in HIV infection. Nutr Rev 68(11):671-681

Straliotto MR, Mancini G, De Oliveira J et al (2010) Acute exposure of rabbits to diphenyl diselenide: a toxicological evaluation. J Appl Toxicol 30(8):761-768

Straliotto MR, de Oliveira J, Mancini G et al (2013) Disubstituted diaryl diselenides as potential atheroprotective compounds: Involvement of TrxR and GPx-like systems. Eur J Pharm Sci 48(4-5):717-725

Straliotto MR, Hort MA, Fiuza B et al (2013) Diphenyl diselenide modulates oxLDL-induced cytotoxicity in macrophage by improving the redox signaling. Biochimie 95(8):1544-1551

Tabuchi Y, Kurebayashi Y (1993) Antisecretory and antiulcer effects of ebselen, a seleno-organic compound, in rats. Jpn J Pharmacol 61(3):255-257

Tabuchi Y, Ogasawara T, Furuhama K (1994) Mechanism of the inhibition of hog gastric $\mathrm{H}+, \mathrm{K}(+)$-ATPase by the seleno-organic compound ebselen. Arzneimittelforschung 44(1):51-54

Tabuchi Y, Sugiyama N, Horiuchi T, Furusawa M, Furuhama K (1995) Ebselen, a seleno-organic compound, protects against ethanolinduced murine gastric mucosal injury in both in vivo and in vitro systems. Eur J Pharmacol 272(2-3):195-201

Takahashi K, Suzuki N, Ogra Y (2020) Effect of gut microflora on nutritional availability of selenium. Food Chem 319:126537

Talas ZS, Gok Y, Ozdemir I, Ates B, Gunal S, Yilmaz I (2015) Synthesis, antioxidant and anti-microbial properties of two organoselenium compounds. Pak J Pharm Sci 28(2):611-616

Tan SM, Sharma A, Yuen DY et al (2013) The modified selenenyl amide, M-hydroxy ebselen, attenuates diabetic nephropathy and diabetes-associated atherosclerosis in ApoE/GPx1 double knockout mice. PLoS ONE 8(7):e69193

Tan S, Sharma A, Stefanovic N, de Haan J (2015) Late-intervention study with ebselen in an experimental model of type 1 diabetic nephropathy. Free Radic Res 49(3):219-227

Tan HW, Mo H-Y, Lau AT, Xu Y-M (2019) Selenium species: Current status and potentials in cancer prevention and therapy. Int J Mol Sci 20(1):75 
Tanaka T, Reddy B, Elbayoumy K (1985) Selenium in chemoprevention of carcinogenesis 3 Inhibition by dietary organoselenium, para-methoxybenzeneselenol, of hepatocarcinogenesis induced by azoxymethane in rats. Jpn J Cancer Res 76(6):462-467

Tang J-Y, Wang L-Q, Jia G et al (2019) The hydroxy-analogue of selenomethionine alleviated lipopolysaccharide-induced inflammatory responses is associated with recover expression of several selenoprotein encoding genes in the spleens of Kunming mice. RSC Adv 9(69):40462-40470

Thabet NM, Moustafa EM (2017) Synergistic effect of Ebselen and gamma radiation on breast cancer cells. Int J Radiat Biol 93(8):784-792

Thangamani S, Younis W, Seleem MN (2015) Repurposing clinical molecule ebselen to combat drug resistant pathogens. PLoS ONE 10(7):e0133877

Thangamani S, Younis W, Seleem MN (2015) Repurposing ebselen for treatment of multidrug-resistant staphylococcal infections. Sci Rep 5:11596

Thangamani S, Eldesouky HE, Mohammad H et al (1861) Ebselen exerts antifungal activity by regulating glutathione (GSH) and reactive oxygen species (ROS) production in fungal cells. Biochim Biophys Acta (BBA) Gen Subj 1:3002-3010

Thenin-Houssier S, De Vera IMS, Pedro-Rosa L et al (2016) Ebselen, a small-molecule capsid inhibitor of HIV-1 replication. Antimicrob Agents Chemother 60(4):2195-2208

Thomas J, Dong Z, Dehaen W, Smet M (2012) Selenium/telluriumcontaining hyperbranched polymers: effect of molecular weight and degree of branching on glutathione peroxidase-like activity. Macromol Rapid Commun 33(24):2127-2132

Thomas AG, Rojas C, Tanega C et al (2013) Kinetic characterization of ebselen, chelerythrine and apomorphine as glutaminase inhibitors. Biochem Biophys Res Commun 438(2):243-248

Thomas SP, Satheeshkumar K, Mugesh G, Row TG (2015) Unusually short chalcogen bonds involving organoselenium: insights into the Se-N bond cleavage mechanism of the antioxidant ebselen and analogues. Chem A Eur J 21(18):6793-6800

Thomson CD, Rea HM, Doesburg VM, Robinson MF (1977) Selenium concentrations and glutathione peroxidase activities in whole blood of New Zealand residents. Br J Nutr 37(3):457-460

Thomson CD, Robinson MF, Campbell DR, Rea HM (1982) Effect of prolonged supplementation with daily supplements of selenomethionine and sodium selenite on glutathione peroxidase activity in blood of New Zealand residents. Am J Clin Nutr 36(1):24-31

Tiegs G, Küsters S, Künstle G, Hentze H, Kiemer AK, Wendel A (1998) Ebselen protects mice against T cell-dependent, TNF-mediated apoptotic liver injury1. J Pharmacol Exp Ther 287(3):1098-1104

Tiwary AK, Stegelmeier BL, Panter KE, James LF, Hall JO (2006) Comparative toxicosis of sodium selenite and selenomethionine in lambs. J Vet Diagn Investig 18(1):61-70

Tsen C, Tappel A (1958) Catalytic oxidation of glutathione and other sulfhydryl compounds by selenite. Catalyst 13(9.6):8.8

Ueno H, Shimizu R, Okuno T et al (2014) Effects of administering sodium selenite, methylseleninic acid, and seleno-L-methionine on glucose tolerance in a streptozotocin/nicotinamide-induced diabetic mouse model. Biol Pharm Bull 37(9):1569-1574

Ueno H, Shimizu R, Okuno T et al (2018) Effect of seleno-l-methionine on oxidative stress in the pancreatic islets of a short-term induced diabetic mouse model in insufficient selenium status. Biol Pharm Bull 41(1):80-85

Unni E, Singh U, Ganther HE, Sinha R (2001) Se-methylselenocysteine activates caspase- 3 in mouse mammary epithelial tumor cells in vitro. BioFactors 14(1-4):169-177

Unni E, Koul D, Yung W-KA, Sinha R (2005) Se-methylselenocysteine inhibits phosphatidylinositol 3-kinase activity of mouse mammary epithelial tumor cells in vitro. Breast Cancer Res 7(5):1-9
Unsal C, Oran M, Albayrak Y et al (2016) Neuroprotective effect of ebselen against intracerebroventricular streptozotocin-induced neuronal apoptosis and oxidative stress in rats. Toxicol Ind Health 32(4):730-740

Ursini F, Maiorino M, Gregolin C (1985) The selenoenzyme phospholipid hydroperoxide glutathione peroxidase. Biochim Biophys Acta (BBA) Gen Subj 839(1):62-70

Uzma N, Kumar BS, Priyadarsini K (2011) Hepatoprotective, immunomodulatory, and anti-inflammatory activities of selenocystine in experimental liver injury of rats. Biol Trace Elem Res 142(3):723-734

Vartak R, Patki M, Menon S et al (2020) $\beta$-cyclodextrin polymer/ Soluplus ${ }^{\circledR}$ encapsulated Ebselen ternary complex (EßpolySol) as a potential therapy for vaginal candidiasis and pre-exposure prophylactic for HIV. Int J Pharm 589:119863

Vartak R, Menon S, Patki M, Billack B, Patel K (2020) Ebselen nanoemulgel for the treatment of topical fungal infection. Eur J Pharm Sci 148:105323

Venturini TP, Chassot F, Loreto ÉS et al (2016) Antifungal activities of diphenyl diselenide and ebselen alone and in combination with antifungal agents against Fusarium spp. Sabouraudia 54(5):550-555

Victoria FN, Anversa R, Penteado F, Castro M, Lenardão EJ, Savegnago L (2014) Antioxidant and antidepressant-like activities of semi-synthetic $\alpha$-phenylseleno citronellal. Eur J Pharmacol 742:131-138

Vinceti M, Chiari A, Eichmüller M et al (2017) A selenium species in cerebrospinal fluid predicts conversion to Alzheimer's dementia in persons with mild cognitive impairment. Alzheimer's Res Ther 9(1):100

Vinceti M, Filippini T, Del Giovane C et al (2018) Selenium for preventing cancer. Cochrane Database Syst Rev 1:1-229

Vinceti M, Filippini T, Malagoli C et al (2019) Amyotrophic lateral sclerosis incidence following exposure to inorganic selenium in drinking water: a long-term follow-up. Environ Res 179:108742

Vinceti M, Chawla R, Filippini T et al (2019) Blood pressure levels and hypertension prevalence in a high selenium environment: results from a cross-sectional study. Nutr Metab Cardiovasc Dis 29(4):398-408

Wall G, Chaturvedi AK, Wormley FL et al (2018) Screening a repurposing library for inhibitors of multidrug-resistant $\mathrm{Can}$ dida auris identifies ebselen as a repositionable candidate for antifungal drug development. Antimicrob agents Chemother 62(10):e01084-18

Wang JF, Komarov P, Sies H, de Groot H (1992) Inhibition of superoxide and nitric oxide release and protection from reoxygenation injury by Ebselen in rat Kupffer cells. Hepatology 15(6):1112-1116

Wang L, Bonorden MJ, Li G-x et al (2009) Methyl-selenium compounds inhibit prostate carcinogenesis in the transgenic adenocarcinoma of mouse prostate model with survival benefit. Cancer Prev Res 2(5):484-495

Wang Y, Zhan X, Zhang X, Wu R, Yuan D (2011) Comparison of different forms of dietary selenium supplementation on growth performance, meat quality, selenium deposition, and antioxidant property in broilers. Biol Trace Elem Res 143(1):261-273

Wang L, Fu J-N, Wang J-Y et al (2011) Selenium-containing thioredoxin reductase inhibitor ethaselen sensitizes non-small cell lung cancer to radiotherapy. Anticancer Drugs 22(8):732-740

Wang L, Yang Z, Fu J et al (2012) Ethaselen: a potent mammalian thioredoxin reductase 1 inhibitor and novel organoselenium anticancer agent. Free Radic Biol Med 52(5):898-908

Wang X, Yun J-W, Lei XG (2014) Glutathione peroxidase mimic ebselen improves glucose-stimulated insulin secretion in murine islets. Antioxid Redox Signal 20(2):191-203 
Wang Y, Gao X, Pedram P et al (2016) Significant beneficial association of high dietary selenium intake with reduced body fat in the CODING study. Nutrients 8(1):24

Wang Z, Li W, Wang Y, Li X, Huang L, Li X (2016) Design, synthesis and evaluation of clioquinol-ebselen hybrids as multitarget-directed ligands against Alzheimer's disease. RSC Adv 6(9):7139-7158

Wang Y, Wallach J, Duane S et al (2017) Developing selective histone deacetylases (HDACs) inhibitors through ebselen and analogs. Drug Des Dev Ther 11:1369

Wang Y, Lin M, Gao X et al (2017) High dietary selenium intake is associated with less insulin resistance in the Newfoundland population. PLoS ONE 12(4): 0174149

Wang W, Fb M, Zx W, Li X, Ds Z (2018) Selenocysteine inhibits human osteosarcoma cells growth through triggering mitochondrial dysfunction and ROS-mediated p53 phosphorylation. Cell Biol Int 42(5):580-588

Wang X-J, Wang M-H, Fu X-T et al (2018) Selenocysteine antagonizes oxygen glucose deprivation-induced damage to hippocampal neurons. Neural Regener Res 13(8):1433

Wang P, Wang J, Xie Z et al (2020) Depletion of multidrug-resistant uropathogenic Escherichia coli BC1 by ebselen and silver ion. $\mathrm{J}$ Cell Mol Med 24:13139-13150

Wasser S, Lim GY, Ong CN, Tan CE (2001) Anti-oxidant ebselen causes the resolution of experimentally induced hepatic fibrosis in rats. J Gastroenterol Hepatol 16(11):1244-1253

Watkins JC, Jane DE (2006) The glutamate story. Br J Pharmacol 147(S1):S100-S108

Watson J (2013) Oxidants, antioxidants and the current incurability of metastatic cancers. Open Biol 3(1): 120144

Watson-Williams E (1919) A preliminary note on the treatment of inoperable carcinoma with selenium. BMJ 2(3067):463

Weekley CM, Aitken JB, Musgrave IF, Harris HH (2012) Methylselenocysteine treatment leads to diselenide formation in human cancer cells: evidence from X-ray absorption spectroscopy studies. Biochemistry 51(3):736-738

Wei L, Zhang Y, Yang C, Wang Q, Zhuang Z, Sun Z (2014) Neuroprotective effects of ebselen in traumatic brain injury model: involvement of nitric oxide and p38 mitogen-activated protein kinase signalling pathway. Clin Exp Pharmacol Physiol 41(2):134-138

Weil R (1915) Chemotherapy and Tumors. J Am Med Assoc 64(16):1283-1289

Weisberger AS, Suhrland LG (1956) Studies on analogues of L-cysteine and L-cystine: III. The effect of selenium cystine on leukemia. Blood 11(1):19-30

Wendel A, Tiegs G (1986) A novel biologically active seleno-organic compound - VI: protection by ebselen (PZ 51) against galactosamine/endotoxin-induced hepatitis in mice. Biochem Pharmacol 35(13):2115-2118

Whanger P (2004) Selenium and its relationship to cancer: an update. Br J Nutr 91(1):11-28

Wilhelm EA, Jesse CR, Leite MR, Nogueira CW (2009) Studies on preventive effects of diphenyl diselenide on acetaminophen-induced hepatotoxicity in rats. Pathophysiology 16(1):31-37

Wilhelm E, Jesse C, Nogueira C (2009) Protective effect of p-methoxyl-diphenyl diselenide in lethal acute liver failure induced by lipopolysaccharide and d-galactosamine in mice. Fundam Clin Pharmacol 23(6):727-734

Wilhelm EA, Jesse CR, Nogueira CW, Savegnago L (2009) Introduction of trifluoromethyl group into diphenyl diselenide molecule alters its toxicity and protective effect against damage induced by 2-nitropropane in rats. Exp Toxicol Pathol 61(3):197-203

Wilhelm EA, Jesse CR, Prigol M, Alves D, Schumacher RF, Nogueira CW (2010) 3-Alkynyl selenophene protects against carbon-tetrachloride-induced and 2-nitropropane-induced hepatic damage in rats. Cell Biol Toxicol 26(6):569-577

Wilhelm EA, Jesse CR, Bortolatto CF, Nogueira CW (2011) (E)2-benzylidene-4-phenyl-1, 3-diselenole has antioxidant and hepatoprotective properties against oxidative damage induced by 2-nitropropane in rats. Fundam Clin Pharmacol 25(1):80-90

Wilhelm EA, Bortolatto CF, Nogueira CW (2012) p-Methoxyl-diphenyl diselenide protects against cisplatin-induced renal toxicity in mice. Food Chem Toxicol 50(5):1187-1193

Wirth T (2015) Small organoselenium compounds: more than just glutathione peroxidase mimics. Angew Chem Int Ed 54(35):10074-10076

Wu G, Li Z, Ju W, Yang X, Fu X, Gao X (2018) Cross-sectional study: relationship between serum selenium and hypertension in the Shandong Province of China. Biol Trace Elem Res 185(2):295-301

Wu W, Yang Z, Xiao X et al (2020) A thioredoxin reductase inhibitor ethaselen induces growth inhibition and apoptosis in gastric cancer. J Cancer 11(10):3013

Xie L, Zheng W, Xin N, Xie J-W, Wang T, Wang Z-Y (2012) Ebselen inhibits iron-induced tau phosphorylation by attenuating DMT1 up-regulation and cellular iron uptake. Neurochem Int 61(3):334-340

Xie Y, Tan Y, Zheng Y, Du X, Liu Q (2017) Ebselen ameliorates $\beta$-amyloid pathology, tau pathology, and cognitive impairment in triple-transgenic Alzheimer's disease mice. J Biol Inorg Chem 22(6):851-865

Xie Y, Liu Q, Zheng L et al (2018) Se-methylselenocysteine ameliorates neuropathology and cognitive deficits by attenuating oxidative stress and metal dyshomeostasis in Alzheimer model mice. Mol Nutr Food Res 62(12): 1800107

Xie L, Yu D, Hu J et al (2018) DMT1 inhibitor ebselen inhibits ironinduced amyloidogenic APP processing. Int J Clin Exp Med 11(8):7907-7916

Xiong S, Markesbery WR, Shao C, Lovell MA (2007) Seleno-L-methionine protects against $\beta$-amyloid and iron/hydrogen peroxidemediated neuron death. Antioxid Redox Signal 9(4):457-467

Xiong YM, Mo XY, Zou X et al (2010) Association study between polymorphisms in selenoprotein genes and susceptibility to Kashin-Beck disease. Osteoarthritis Cartil 18(6):817-824

$\mathrm{Xu}$ L, Gong C, Li G et al (2018) Ebselen suppresses inflammation induced by Helicobacter pylori lipopolysaccharide via the p38 mitogen-activated protein kinase signaling pathway. Mol Med Rep 17(5):6847-6851

Ünlü YA, Ekrem K, Hafize U et al (2002) The neuroprotective effects of ebselen in experimental spinal cord injury. Turk Neurosurg 12(1-2):9-16

Yamaguchi T, Sano K, Takakura K et al (1998) Ebselen in acute ischemic stroke: a placebo-controlled, double-blind clinical trial. Stroke 29(1):12-17

Yamakawa GR, Eyolfson E, Weerawardhena H, Mychasiuk R (2020) Administration of diphenyl diselenide ( $\mathrm{PhSe}) 2$ following repetitive mild traumatic brain injury exacerbates anxiety-like symptomology in a rat model. Behav Brain Res 382:112472

Yan J, Pang Y, Zhuang J et al (2019) Selenepezil, a selenium-containing compound, exerts neuroprotective effect via modulation of the Keap1-Nrf2-ARE pathway and attenuates A $\beta$-induced cognitive impairment in vivo. ACS Chem Neurosci 10(6):2903-2914

Yang H, Jia X (2014) Safety evaluation of Se-methylselenocysteine as nutritional selenium supplement: acute toxicity, genotoxicity and subchronic toxicity. Regul Toxicol Pharmacol 70(3):720-727

Yang Y, Yang M, Ai F, Huang C (2017) Cardioprotective effect of Aloe vera biomacromolecules conjugated with selenium trace element on myocardial ischemia-reperfusion injury in Rats. Biol Trace Elem Res 177(2):345-352 
Yarmolinsky J, Bonilla C, Haycock PC et al (2018) Circulating selenium and prostate cancer risk: a mendelian randomization analysis. JNCI J Natl Cancer Inst 110(9):1035-1038

Ye S, Zheng X, Hu T, Zeng H (2016) Synthesis, SAR and biological evaluation of a novel series of 1-(2-chloroethyl)-1-nitroso-3-(2(3-oxobenzoelenazol-2 (3H)-yl) ethyl) urea: organoselenium compounds for cancer therapy. Cell Mol Biol (Noisy-le-grand) 62(7):6-14

Ye S-f, Li J, Ji S-m, Zeng H-h, Lu W (2017) Dose-biomarker-response modeling of the anti-cancer effect of ethaselen in a human nonsmall cell lung cancer xenograft mouse model. Acta Pharmacol Sin 38(2):223-232

Yoshida M, Iizuka K, Terada A et al (2000) Prevention of nephrotoxicity of cisplatin by repeated oral administration of ebselen in rats. Tohoku J Exp Med 191(4):209-220

Yu Y, Jin Y, Zhou J et al (2017) Ebselen: mechanisms of glutamate dehydrogenase and glutaminase enzyme inhibition. ACS Chem Biol 12(12):3003-3011

Yu S-C, Ri D-M, Kühn H (2018) Hydrophobicity and glutathione peroxidase-like activity of substituted salicyloyl-5-seleninic acids: Re-investigations on aromatic selenium compounds based on their hydrophobicity. J Organomet Chem 862:86-94

Zamberlan D, Arantes L, Machado M, Golombieski R, Soares F (2014) Diphenyl-diselenide suppresses amyloid- $\beta$ peptide in Caenorhabditis elegans model of Alzheimer's disease. Neuroscience 278:40-50

Zasso FB, Goncales CE, Jung EA et al (2005) On the mechanisms involved in antinociception induced by diphenyl diselenide. Environ Toxicol Pharmacol 19(2):283-289

Zborowski VA, Sari MH, Heck SO et al (2016) $p$-Chloro-diphenyl diselenide reverses memory impairment-related to stress caused by corticosterone and modulates hippocampal $[3 \mathrm{H}]$ glutamate uptake in mice. Physiol Behav 164:25-33

Zborowski VA, Heck SO, Vencato M, Pinton S, Marques LS, Nogueira CW (2020) Keap1/Nrf2/HO-1 signaling pathway contributes to $p$-chlorodiphenyl diselenide antidepressant-like action in diabetic mice. Psychopharmacology 237(2):363-374

Zembowicz A, Hatchett RJ, Radziszewski W, Gryglewski RJ (1993) Inhibition of endothelial nitric oxide synthase by ebselen. Prevention by thiols suggests the inactivation by ebselen of a critical thiol essential for the catalytic activity of nitric oxide synthase. J Pharmacol Exp Ther 267(3):1112-1118

Zeng H, Combs GF Jr (2008) Selenium as an anti-cancer nutrient: roles in cell proliferation and tumor cell invasion. J Nutr Biochem 19(1):1-7

Zeng H, Yan L, Cheng W-H, Uthus EO (2011) Dietary selenomethionine increases exon-specific DNA methylation of the $\mathrm{p} 53$ gene in rat liver and colon mucosa. J Nutr 141(8):1464-1468

Zhan Y, Cao B, Qi Y et al (2013) Methylselenol prodrug enhances MDV3100 efficacy for treatment of castration-resistant prostate cancer. Int J Cancer 133(9):2225-2233

Zhang M, Nomura A, Uchida Y et al (2002) Ebselen suppresses late airway responses and airway inflammation in guinea pigs. Free Radic Biol Med 32(5):454-464

Zhang Z-H, Chen C, Wu Q-Y et al (2016) Selenomethionine reduces the deposition of beta-amyloid plaques by modulating $\beta$-secretase and enhancing selenoenzymatic activity in a mouse model of Alzheimer's disease. Metallomics 8(8):782-789

Zhang Z-H, Chen C, Wu Q-Y et al (2016) Selenomethionine ameliorates neuropathology in the olfactory bulb of a triple transgenic mouse model of Alzheimer's disease. Int J Mol Sci 17(10):1595

Zhang Z-H, Wu Q-Y, Zheng R et al (2017) Selenomethionine mitigates cognitive decline by targeting both tau hyperphosphorylation and autophagic clearance in an Alzheimer's disease mouse model. J Neurosci 37(9):2449-2462
Zhang Z-H, Wu Q-Y, Chen C et al (2017) Selenomethionine attenuates the Amyloid- $\beta$ level by both inhibiting Amyloid- $\beta$ production and modulating autophagy in neuron-2a/A $\beta$ PPswe cells. J Alzheimer's Dis 59(2):591-602

Zhang Z-H, Wu Q-Y, Chen C et al (2018) Comparison of the effects of selenomethionine and selenium-enriched yeast in the tripletransgenic mouse model of Alzheimer's disease. Food Funct 9(7):3965-3973

Zhang J, Taylor EW, Bennett K, Saad R, Rayman MP (2020) Association between regional selenium status and reported outcome of COVID-19 cases in China. Am J Clin Nutr 111(6):1297-1299

Zhang D-W, Yan H-L, Xu X-S et al (2020) The selenium-containing drug ebselen potently disrupts LEDGF/p75-HIV-1 integrase interaction by targeting LEDGF/p75. J Enzyme Inhib Med Chem 35(1):906-912

Zhang Y, Cartland SP, Henriquez R et al (2020) Selenomethionine supplementation reduces lesion burden, improves vessel function and modulates the inflammatory response within the setting of atherosclerosis. Redox Biol 29:101409

Zhang X, Peng K, Zhang X (2020) The function of the NMDA receptor in hypoxic-ischemic encephalopathy. Front Neurosci 14:998

Zhang J, Saad R, Taylor EW, Rayman MP (2020b) Selenium and selenoproteins in viral infection with potential relevance to COVID19. Redox Biol 37:101715

Zhang Z, Chen C, Jia S et al (2020f) Selenium restores synaptic deficits by modulating NMDA receptors and selenoprotein $\mathrm{K}$ in an Alzheimer's disease model. Antioxidants Redox Signal (JA). https:// doi.org/10.1089/ars.2019.7990

Zhao H, Whitfield ML, Xu T, Botstein D, Brooks JD (2004) Diverse effects of methylseleninic acid on the transcriptional program of human prostate cancer cells. Mol Biol Cell 15(2):506-519

Zheng R, Zhang Z-H, Chen C et al (2017) Selenomethionine promoted hippocampal neurogenesis via the PI3K-Akt-GSK3 $\beta$-Wnt pathway in a mouse model of Alzheimer's disease. Biochem Biophys Res Commun 485(1):6-15

Zheng X, Xu W, Sun R, Yin H, Dong C, Zeng H (2017) Synergism between thioredoxin reductase inhibitor ethaselen and sodium selenite in inhibiting proliferation and inducing death of human non-small cell lung cancer cells. Chem Biol Interact 275:74-85

Zheng L, Feng L, Jiang W-D et al (2018) Selenium deficiency impaired immune function of the immune organs in young grass carp (Ctenopharyngodon idella). Fish Shellfish Immunol 77:53-70

Zheng R, Zhang Z-H, Zhao Y-X et al (2019) Transcriptomic insights into the response of the olfactory bulb to selenium treatment in a mouse model of Alzheimer's disease. Int J Mol Sci 20(12):2998

Zheng X, Chen Y, Bai M et al (2019) The antimetastatic effect and underlying mechanisms of thioredoxin reductase inhibitor ethaselen. Free Radic Biol Med 131:7-17

Zhou J, Huang K, Lei XG (2013) Selenium and diabetes-evidence from animal studies. Free Radic Biol Med 65:1548-1556

Zhou J, Xu H, Huang K (2016) Organoselenium small molecules and chromium (III) complexes for intervention in chronic lowgrade inflammation and type 2 diabetes. Curr Top Med Chem 16(8):823-834

Zmudzinski M, Rut W, Olech K, et al (2020) Ebselen derivatives are very potent dual inhibitors of SARS-CoV-2 proteases-PLpro and Mpro in in vitro studies. bioRxiv 273979

Publisher's Note Springer Nature remains neutral with regard to jurisdictional claims in published maps and institutional affiliations. 